\title{
Exploring the X-ray sky with the XMM-Newton bright serendipitous survey $\star, \star \star$
}

\author{
R. Della Ceca ${ }^{1}$, T. Maccacaro ${ }^{1}$, A. Caccianiga ${ }^{1}$, P. Severgnini ${ }^{1}$, V. Braito ${ }^{1}$, X. Barcons ${ }^{2}$, F. J. Carrera ${ }^{2}$, \\ M. G. Watson ${ }^{3}$, J. A. Tedds ${ }^{3}$, H. Brunner ${ }^{4}$, I. Lehmann ${ }^{4}$, M. J. Page ${ }^{5}$, G. Lamer ${ }^{6}$, and A. Schwope ${ }^{6}$ \\ 1 INAF-Osservatorio Astronomico di Brera, via Brera 28, 20121 Milano, Italy \\ e-mail: [rdc; tommaso; caccia;paola; braito]@brera.mi.astro.it \\ 2 Instituto de Fisica de Cantabria (CSIC-UC), Avenida de los Castros, 39005 Santander, Spain \\ e-mail: [barcons; carreraf]@ifca.unican.es \\ 3 X-ray Astronomy Group, Department of Physics and Astronomy, Leicester University, Leicester LE1 7RH, UK \\ e-mail: [mgw; jat]@star.le.ac.uk \\ 4 Max-Planck-Institut für Extraterrestrische Physik, Postfach 1312, 85741 Garching, Germany \\ e-mail: [hbrunnerl;ile] @mpe.mpg.de \\ 5 Mullard Space Science Laboratory, University College London, Holmbury St. Mary, Dorking, Surrey RH5 6NT, UK \\ e-mail: mjp@mssl.ucl.ac.uk \\ 6 Astrophysikalisches Institut Potsdam (AIP), An der Sternwarte 16, 14482 Potsdam, Germany \\ e-mail: [glamer; aschwope]@aip.de
}

Received 12 February 2004 / Accepted 14 July 2004

\begin{abstract}
We present here "The XMM-Newton Bright Serendipitous Survey", composed of two flux-limited samples: the XMM-Newton Bright Source Sample (BSS, hereafter) and the XMM-Newton "Hard" Bright Source Sample (HBSS, hereafter) having a flux limit of $f_{x} \simeq 7 \times 10^{-14} \mathrm{erg} \mathrm{cm}^{-2} \mathrm{~s}^{-1}$ in the $0.5-4.5 \mathrm{keV}$ and $4.5-7.5 \mathrm{keV}$ energy band, respectively. After discussing the main goals of this project and the survey strategy, we present the basic data on a complete sample of $400 \mathrm{X}$-ray sources (389 of them belong to the BSS, 67 to the HBSS with 56 X-ray sources in common) derived from the analysis of 237 suitable XMM-Newton fields ( 211 for the HBSS). At the flux limit of the survey we cover a survey area of 28.10 ( 25.17 for the HBSS) sq. deg. The extragalactic number-flux relationships (in the $0.5-4.5 \mathrm{keV}$ and in the $4.5-7.5 \mathrm{keV}$ energy bands) are in good agreement with previous and new results making us confident about the correctness of data selection and analysis. Up to now $\sim 71 \%$ ( $\sim 90 \%)$ of the sources have been spectroscopically identified making the BSS (HBSS) the sample with the highest number of identified XMM-Newton sources published so far. At the X-ray flux limits of the sources studied here we found that: a) the optical counterpart in the majority ( $\sim 90 \%)$ of cases has a magnitude brighter than the POSS II limit $\left(R \sim 21^{\mathrm{mag}}\right)$; b) the majority of the objects identified so far are broad line AGN both in the BSS and in the HBSS. No obvious trend of the source spectra (as deduced from the Hardness Ratios analysis) as a function of the count rate is measured and the average spectra of the "extragalactic" population corresponds to a $(0.5-4.5 \mathrm{keV})$ energy spectral index of $\sim 0.8(\sim 0.64)$ for the BSS (HBSS) sample. Based on the hardness ratios we infer that about 13\% (40\%) of the sources in the BSS (HBSS) sample are described by an energy spectral index flatter than that of the cosmic X-ray background. Based on previous X-ray spectral results on a small subsample of objects we speculate that all these sources are indeed absorbed AGN with the $N_{\mathrm{H}}$ ranging from a few times $10^{21}$ up to few times $10^{23} \mathrm{~cm}^{-2}$. We do not find strong evidence that the $4.5-7.5 \mathrm{keV}$ survey is sampling a completely different source population if compared with the $0.5-4.5 \mathrm{keV}$ survey; rather we find that, as expected from the CXB synthesis models, the hard survey is simply picking up a larger fraction of absorbed AGN. At the flux limit of the HBSS sample we measure surface densities of optically type 1 and type 2 AGN of $1.63 \pm 0.25 \mathrm{deg}^{-2}$ and $0.83 \pm 0.18 \mathrm{deg}^{-2}$, respectively; optically type 2 AGN represent $34 \pm 9 \%$ of the total AGN population. Finally, we have found a clear separation, in the hardness ratio diagram and in the (hardness ratio) vs. (X-ray to optical flux ratio) diagram, between Galactic "coronal emitting" stars and extragalactic sources. The information and "calibration" reported in this paper will make the existing and incoming XMM-Newton catalogs a unique resource for astrophysical studies.
\end{abstract}

Key words. X-rays: diffuse background - surveys - X-rays: active galaxies

* Based on observations obtained with XMM-Newton, an ESA science mission with instruments and contributions directly funded by ESA Member States and the USA (NASA). The majority of the new optical spectroscopy data used here have been obtained using the facilities of the Italian "Telescopio Nazionale Galileo" (TNG) and of the European Southern Observatory (ESO).

$\star \star$ Tables 2-4 and Appendices are only available in electronic form at http://www. edpsciences.org 


\section{Introduction}

Deep Chandra and XMM-Newton observations (Brandt et al. 2001; Rosati et al. 2002; Moretti et al. 2003; Hasinger et al. 2001; Alexander et al. 2003) have recently resolved $>\sim 80 \%$ of the 2-10 keV cosmic X-ray background (CXB) into discrete sources down to $f_{x} \sim 3 \times 10^{-16} \mathrm{erg} \mathrm{cm}^{-2} \mathrm{~s}^{-1}$.

The statistical analysis (stacked spectra and hardness ratios) performed on these faint samples provide information on the X-ray spectral properties of the sources making up most of the CXB. The X-ray data are consistent with AGN being the dominant contributors of the CXB (see Brandt et al. 2004, and reference therein) and, as inferred by the X-ray colors, a significant fraction of these sources have hard, presumably obscured, $\mathrm{X}$-ray spectra, in agreement with the predictions of CXB synthesis models (see Setti \& Woltjer 1989; Madau et al. 1994; Comastri et al. 1995, 2001; Gilli et al. 2001; Ueda et al. 2003).

However the majority of the sources found in these medium to deep fields are too faint to provide good X-ray spectral information. Furthermore, the extremely faint magnitude of a large number of their optical counterparts makes the spectroscopic identifications very difficult, or even impossible, with the present day ground-based optical telescopes.

Thus, notwithstanding the remarkable results obtained by reaching very faint $\mathrm{X}$-ray fluxes, the broad-band physical properties (e.g. the relationship between optical absorption and X-ray obscuration and the reason why AGN with similar X-ray properties have completely different optical appearance) are not yet completely understood. A step forward toward the solution of these problems has been recently obtained by Mainieri et al. (2002); Piconcelli et al. (2002, 2003); Georgantopoulos et al. (2004); Caccianiga et al. (2004) and Perola et al. (2004) using samples of serendipitous sources for which medium/good quality XMM-Newton and optical data are available.

With the aim of complementing the results obtained by medium to deep X-ray surveys, the XMM-Newton Survey Science Centre" (SSC) has conceived the "XMM-Newton Bright Serendipitous Survey". This survey comprises two high galactic latitude $\left(|b|>20^{\circ}\right)$, flux limited samples of serendipitous XMM-Newton sources: the XMM Bright Source Sample (hereafter BSS) and the XMM Hard Bright Source Sample (hereafter HBSS) having a flux limit of $f_{x} \simeq 7 \times$ $10^{-14} \mathrm{erg} \mathrm{cm}^{-2} \mathrm{~s}^{-1}$ in the $0.5-4.5 \mathrm{keV}$ and $4.5-7.5 \mathrm{keV}$ energy bands, respectively. In addition to the issues related to the CXB, where is now largely accepted that X-ray obscured AGNs play a significant (and perhaps major) role, the use of the 4.5-7.5 keV energy band partially reduces the strong bias against absorbed sources which occurs when selecting at softer energies (or when selecting in the optical domain), and is therefore fundamental to study the accretion history in the Universe (see e.g. Fiore et al. 2003). A similar energy selection band (i.e.

\footnotetext{
1 The XMM-Newton Survey Science Centre is an international collaboration involving a consortium of 10 institutions appointed by ESA to help the SOC in developing the software analysis system, to pipeline process all the XMM-Newton data, and to exploit the XMM-Newton serendipitous detections, see http://xmmssc-www.star.le.ac.uk
}

5-10 keV) was pioneered by the BeppoSAX-HELLAS (Fiore et al. 2001) and the ASCA-SHEEP (Nandra et al. 2003) surveys.

The well defined criteria (completeness, representativeness, etc.) of this sample will allow both a detailed study of individual sources of high interest, and statistical studies of populations. In particular, the BSS and HBSS samples will be fundamental to complement other medium and deep XMM-Newton and Chandra survey programs (having fluxes 10 to 100 times fainter and covering a smaller area of the sky) and will provide a larger baseline for all evolutionary studies. Moreover, the good X-ray statistics which characterize most of the sources in the "XMM-Newton Bright Serendipitous Survey", combined with the relative brightness of their optical counterparts, will allow us to investigate their physical properties in detail. Indeed this sample is already contributing to the solution of some critical open (and "hot") questions like the relationship between optical absorption and X-ray obscuration (Caccianiga et al. 2004) and the physical nature of the "X-ray bright optically normal galaxies" (Severgnini et al. 2003). Many of these issues are investigated with difficulty using the fainter X-ray samples because of their typical poor counts statistics for each source.

The spectroscopic identifications together with the X-ray (spectral, morphological and variability) parameters will be made available to the community and can be used to define statistical identification procedures to select rare and interesting classes of X-ray sources, enabling the application of these procedures to the vast amount of XMM-Newton serendipitous data that will be accumulated during the lifetime of the mission $^{2}$.

In this paper we discuss the BSS and the HBSS survey strategy, we present a complete sample of 400 sources extracted from the analysis of 237 XMM-Newton fields and we discuss some preliminary statistical results based on the spectroscopic identification done so far.

This paper is organized as follows. In Sect. 2 we discuss the survey strategy (e.g. energy selection bands, primary selection camera and criteria for field and source selection), we present basic information on the XMM-Newton fields used and on the sources belonging to the BSS and HBSS samples and we discuss the completeness of the "XMM-Newton Bright Serendipitous Survey". In Sect. 3 we discuss the number-flux relationship, the identification work done so far, the broadband X-ray spectral properties of the sample, the position of

\footnotetext{
${ }^{2}$ One of the responsibilities of the XMM-Newton SSC is the production of the XMM-Newton Source Catalogue. This catalogue will provide a rich and unique resource for generating well-defined samples for specific studies, using the fact that X-ray selection is a highly efficient way of selecting certain types of objects, like for instance AGN, clusters of galaxies and active stars. The first XMM-Newton Serendipitous Source Catalogue (1XMM), released on 2003 April 7, contains source detections drawn from 585 XMM-Newton EPIC observations and a total of 30000 individual $\mathrm{X}$-ray sources having a likelihood value above 8 and good quality flags. The median flux (in the total photon energy band $0.2-12 \mathrm{keV}$ ) of the catalogue sources is $\sim 3 \times 10^{-14} \mathrm{erg} \mathrm{cm}^{-2} \mathrm{~s}^{-1}$, with $\sim 12 \%$ of them having fluxes below $\sim 1 \times 10^{-14} \mathrm{erg} \mathrm{cm}^{-2} \mathrm{~s}^{-1}$, see http://xmmssc-www.star.le.ac.uk/
} 
the sources in the diagram obtained using the X-ray spectral information (provided by the hardness ratio) and the X-ray to optical flux ratio as well as the surface densities $(\log (N>S)-$ $\log S$ ) of optically type 1 and type 2 AGN in the HBSS sample. Finally, the summary and the conclusions are reported in Sect. 4. In the appendices we discuss our approach to evaluate the background quality of the data used and to deal with the Xray sources falling close to the gaps between the CCDs or close to the edge of the CCDs. Throughout this paper $H_{0}=65 \mathrm{~km}$ $\mathrm{s}^{-1} \mathrm{Mpc}^{-1}$ and $\Omega_{\lambda}=0.7, \Omega_{\mathrm{M}}=0.3$ are assumed; the energy spectral index, $\alpha_{\mathrm{E}}$, quoted in this paper refers to a power-law spectral model having $S_{\mathrm{E}} \propto E^{-\alpha_{\mathrm{E}}}$.

\section{Survey strategy and sample(s) selection}

\subsection{Selection energy band(s)}

We have decided to survey the bright X-ray sky in two complementary energy bands: the $0.5-4.5 \mathrm{keV}$ and the $4.5-7.5 \mathrm{keV}$ energy bands.

The choice of the $0.5-4.5 \mathrm{keV}$ energy band is mainly motivated by the desire to avoid the very soft photons (minimizing non-uniformities introduced by the different values of Galactic absorbing column densities along the line of sight) and by the need to compromise between a broad passband (to favor throughput) and a narrow passband (to minimize nonuniformities in the selection function due to different source spectra). Furthermore in the $0.5-4.5 \mathrm{keV}$ band XMM-Newton has the highest throughput.

The choice of the 4.5-7.5 keV energy band (one of the energy bands used in the standard pipeline processing system of the XMM-Newton data) was instead dictated by the need to study the composition of the source population (in terms of observed and intrinsic energy distribution and absorption properties) as a function of the energy selection band, comparing the sources selected in this band with those selected in the softer $0.5-4.5 \mathrm{keV}$ energy range. Moreover this energy band reduces the strong bias against absorbed sources which occurs when selecting at softer energies.

\subsection{Primary selection camera}

The source sample has been defined using the data from the EPIC MOS2 detector only. The main reasons for this choice are:

1. unlike the EPIC pn, the EPIC MOS cameras have a detector pattern that simplifies the analysis of the field. For example, in the case of the EPIC MOS detectors the source target, in the large majority of the observations, is fully contained in the central chip;

2. the PSF in the 2 EPIC MOSs is narrower than in the EPIC pn. In particular, the EPIC MOS2 has the "best" PSF $\left(F W H M \sim 4.4^{\prime \prime}\right.$ and $H E W \sim 13.0^{\prime \prime}$ at $1.5 \mathrm{keV}$, see Ehle et al. 2003). As a comparison, the EPIC pn PSF has FWHM $\sim 6.6^{\prime \prime}$ and $H E W \sim 15.2^{\prime \prime}$, while the EPIC MOS1 PSF has FWHM 4.3" and HEW $\sim 13.8^{\prime \prime}$.
3. the gaps between the EPIC MOS chips are narrower than the gaps in the EPIC pn detector, simplifying source detection and analysis and maximizing the survey area;

4. unlike with the EPIC pn camera, we can still use part of the EPIC MOS2 observations in large- and small-window mode by only excluding the area occupied by the central chip. Since $\sim 25 \%$ of the observations have been performed in window mode, retaining these observations will maximize the searched area, speeding up the creation and definition of the source sample.

The major disadvantage of the EPIC MOS2 camera when compared to the EPIC pn camera is the reduced sensitivity, because of its smaller effective area. However, since the BSS and HBSS samples contain relatively bright sources, and considering the minimum exposure times used here (see Sect. 2.5) this lower efficiency does not affect the source selection of the samples presented here. Obviously, once a source is detected and included in the sample, additional information using data from the EPIC MOS1 and pn detectors are collected to increase the statistics for the X-ray spectra, timing and morphology analysis.

\subsection{Source detection}

Each EPIC MOS2 observation used here (see Table 2) has been processed through the pipeline processing system used for the production of the XMM-Newton Serendipitous Source Catalogue, based on tasks from the XMM-Newton Science Analysis Software. Full details about the processing system, the pipeline products as well as the source searching procedures, flux measurements, source likelihood parameter, corrections for vignetting and PSF, etc. can be found in http://xmmssc-www.star.le.ac.uk. We note that the count rate(s) reported in this paper have been already corrected for vignetting and PSF.

\subsection{Criteria for source selection}

Since the BSS and HBSS samples have been designed to contain relatively bright $\mathrm{X}$-ray sources not all the sources detected in each individual MOS2 field are adequate to be included in these samples. We discuss here the criteria for the BSS and HBSS source selection within each EPIC MOS2 field:

1. BSS sample: $0.5-4.5 \mathrm{keV}$ count-rate $\geq 1 \times 10^{-2} \mathrm{cts} / \mathrm{s}$. At this count rate limit, and given the considered range of MOS2 exposure times (see Table 2 and Fig. 1), all the selected sources have a likelihood parameter in the $0.5-4.5 \mathrm{keV}$ energy band greater than $\sim 18$ (corresponding to a probability for a random Poissonian fluctuation to have caused the observed source counts of $1.5 \times 10^{-8}$ ). No further constraint is thus needed to ensure the source reliability.

HBSS sample: $4.5-7.5 \mathrm{keV}$ count-rate $\geq 2 \times 10^{-3} \mathrm{cts} / \mathrm{s}$ and likelihood parameter in the $4.5-7.5 \mathrm{keV}$ energy band greater than 12 (corresponding to a probability of $6 \times 10^{-6}$ for a spurious detection). 

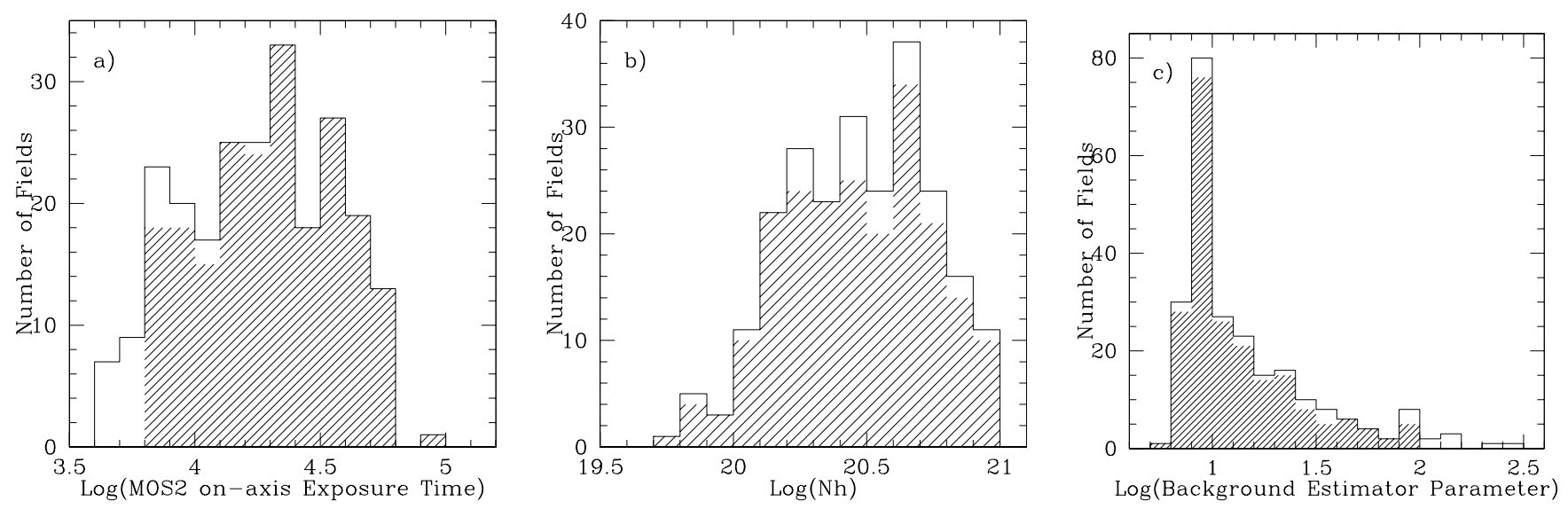

Fig. 1. Histograms of some basic properties of the XMM-Newton MOS2 fields used for the sample selection. Normal histograms refer to the XMM-Newton fields used to define the BSS sample, while shaded histograms refer to the subset of XMM-Newton fields used to define the HBSS sample. Panel a) histogram of the MOS2 on-axis good-time exposure; Panel b) histogram of the Galactic hydrogen column density along the line of sight; Panel c) histogram of the Background Estimator Parameter (see Appendix B for details).

The combination of count-rate limit(s) and likelihood parameter(s) of the sources in the BSS and HBSS samples is such that none of them are expected to be spurious.

The count rate to flux conversion factors (CR2F) depend on the source spectra and in Table 1 we report some CR2F as a function of the input source spectra for a fixed Galactic $N_{\mathrm{H}}$ value of $3 \times 10^{20} \mathrm{~cm}^{-2}$ (corresponding to the median value for the XMM-Newton fields used here).

For a source with a power-law spectrum with energy spectral index $\alpha_{\mathrm{E}}$ between 0.7 and 0.8 the count rate limit in the two chosen bands corresponds to a flux limit of $\sim 7 \times 10^{-14} \mathrm{erg} \mathrm{cm}^{-2} \mathrm{~s}^{-1}$.

2. Sources with a distance from the EPIC MOS2 center between an inner radius $\left(R_{\text {in }}\right)$ and an outer radius $\left(R_{\text {out }}\right)$.

$R_{\text {in }}$ depends on the actual size and brightness of the target and on the window mode. $R_{\text {in }}$ ranges between 0 (e.g. survey fields with no "target") and 8 arcmin (e.g. bright/extended $\mathrm{X}$-ray sources or large- and small-window mode). In this way the area of the detector "obscured" by the presence of the target or not exposed is excluded from the analysis. To guarantee that all the sources in the catalogue are truly serendipitous, the size of $R_{\text {in }}$ has been adapted in order to exclude the target and the sources physically related to the target. $R_{\text {out }}$ is, for the large majority of the fields, equal to $13 \mathrm{arcmin}$. In the few overlapping fields we have excluded from the analysis the outer region of one of the overlapping fields in order to obtain a mosaic of separate and independent regions on the sky. The values of $R_{\text {in }}$ and $R_{\text {out }}$ used for each MOS2 image are listed in Table 2.

3. We have also excluded the sources too close to the edges of the field of view or to the gaps between the CCDs. These sources could have either the flux and/or the source centroid poorly determined (due to the proximity to the edges and/or the gaps), and therefore could degrade the quality of the data, and would require uncertainty corrections thus representing a problem in the subsequent analysis and interpretation of the data. In Appendix A we discuss the procedure used to take into account this problem in an objective way. Obviously, the excluded area has been taken into account in the computation of the sky coverage.

\subsection{Criteria for field selection}

Not all the available EPIC MOS2 pointings are adequate for producing the BSS and HBSS samples. We have defined a set of selection criteria to avoid problematic regions of the sky, to maximize the availability of ancillary information at other frequencies (i.e. optical and radio) and to speed up the optical identification process. The majority of the fields selection criteria are common to the BSS and HBSS; however, as discussed below, we have been more conservative on the minimum exposure time and background properties for the fields used to define the HBSS sample. The criteria adopted for field selection are:

1. availability to SSC before March 2003 (XMM-Newton fields that are public or with PI granted permission);

2. high Galactic latitude $\left(|b| \geq 20^{\circ}\right)$ to avoid crowded fields, to obtain a relatively "clean" extragalactic sample and to have magnitude information for the optical counterparts from the Digital Sky Survey material (the Automated Plate Machine - APM - catalogue ${ }^{3}$ is almost complete for $|b| \geq 20^{\circ}$ );

3. Galactic absorbing column density along the line of sight less than $10^{21} \mathrm{~cm}^{-2}$, to minimize non-uniformities introduced by large values of the Galactic $N_{\mathrm{H}}$;

4. exclusion of fields centered on bright and/or extended X-ray or optical targets and those containing very bright stars in the optical band. In the first two cases the effective area of sky covered and the actual flux limit are difficult to estimate correctly, making the derivation of the skycoverage more uncertain; in the latter case the search for the optical counterpart of the X-ray sources could be very difficult or even impossible due to the presence of the bright star;

\footnotetext{
${ }^{3}$ http://www.ast.cam.ac.uk/ apmcat/
} 
Table 1. MOS2 count rate to flux conversion factors.

\begin{tabular}{rrll}
\hline \hline$\alpha_{\mathrm{E}}$ & HR2 & $\begin{array}{l}\text { Flux }(0.5-4.5 \mathrm{keV}) \\
10^{-12} \mathrm{erg} \mathrm{cm}^{-2} \mathrm{~s}^{-1}\end{array}$ & $\begin{array}{l}\text { Flux }(4.5-7.5 \mathrm{keV}) \\
10^{-11} \mathrm{erg} \mathrm{cm}^{-2} \mathrm{~s}^{-1} \\
(1)\end{array}$ \\
$(2)$ & $(3)$ & $4.53 ; 4.54 ; 4.59$ \\
\hline-3.0 & 0.77 & $14.3 ; 14.4 ; 15.0$ & 4.0 \\
-2.0 & 0.56 & $12.8 ; 12.9 ; 13.6$ & $4.24 ; 4.25 ; 4.30$ \\
-1.0 & 0.22 & $10.6 ; 10.8 ; 11.7$ & $3.97 ; 3.97 ; 4.03$ \\
-0.5 & 0.01 & $9.47 ; 9.61 ; 10.6$ & $3.84 ; 3.84 ; 3.90$ \\
0.0 & -0.22 & $8.35 ; 8.49 ; 9.55$ & $3.71 ; 3.72 ; 3.77$ \\
0.4 & -0.38 & $7.56 ; 7.70 ; 8.82$ & $3.62 ; 3.62 ; 3.68$ \\
0.5 & -0.42 & $7.38 ; 7.53 ; 8.66$ & $3.59 ; 3.60 ; 3.65$ \\
0.7 & -0.49 & $7.06 ; 7.20 ; 8.37$ & $3.55 ; 3.55 ; 3.61$ \\
0.9 & -0.57 & $6.78 ; 6.92 ; 8.11$ & $3.50 ; 3.51 ; 3.56$ \\
1.0 & -0.60 & $6.65 ; 6.79 ; 8.00$ & $3.48 ; 3.49 ; 3.54$ \\
1.5 & -0.74 & $6.16 ; 6.31 ; 7.61$ & $3.38 ; 3.38 ; 3.43$ \\
2.0 & -0.83 & $5.89 ; 6.04 ; 7.46$ & $3.28 ; 3.28 ; 3.33$ \\
3.0 & -0.94 & $5.75 ; 5.94 ; 7.62$ & $3.10 ; 3.10 ; 3.15$ \\
\hline
\end{tabular}

Columns are as follows: (1) Energy spectral index $\left(S_{\mathrm{E}} \propto E^{-\alpha_{\mathrm{E}}}\right)$; (2) corresponding hardness ratio $H R 2$ computed as discussed in Sect. 3.3; (3) Flux (corrected for Galactic absorption) in the $0.5-4.5 \mathrm{keV}$ energy band corresponding to an observed count rate of $1 \mathrm{cts} / \mathrm{s}$ in the same energy band. The three numbers refer to thin, medium and thick filters; (4) Flux (corrected for Galactic absorption) in the $4.5-7.5 \mathrm{keV}$ energy band corresponding to an observed count rate of $1 \mathrm{cts} / \mathrm{s}$ in the same energy band. The three numbers refer to thin, medium and thick filters. NOTE - A Galactic absorbing column density of $3 \times 10^{20} \mathrm{~cm}^{-2}$, the median value for the XMM-Newton fields used here, has been assumed. Given the range of the Galactic absorbing column density along the line of sight (from $\sim 5 \times 10^{19}$ to $10^{21} \mathrm{~cm}^{-2}$ ) the CR2F in the $0.5-4.5 \mathrm{keV}$ energy range are accurate to $\pm 18 \%$; the $\mathrm{CR} 2 \mathrm{~F}$ in the $4.5-7.5 \mathrm{keV}$ energy range are independent of the Galactic $N_{\mathrm{H}}$. Please note that in the case of sources having extreme $H R 2$ values the observed spectra could be much more complex than a simple power law; for these sources the conversion factor reported in the table should be considered only as indicative and a proper X-ray spectral analysis is needed.

5. exclusion of fields south of Dec $=-80$ deg since it could be very difficult to obtain good quality spectroscopy given the location of the optical facilities available to us;

6. good-time interval ${ }^{4}$ exposure $>\sim 5 \mathrm{ks}$ for the BSS and $\geq 7 \mathrm{ks}$ for the HBSS. According to the results presented and discussed below, with these constraints all the sources in the two samples are detectable across the whole field of view considered, ensuring a "flat" sensitivity and therefore a flat sky coverage at the sampled fluxes.

7. finally, we have also excluded EPIC MOS2 pointings suffering from a high background rate (i.e. accumulated during particle background flares). The background restriction has been more conservative for the set of fields that have been used to define the HBSS sample since the overall background is more critical given the faintness of the sources in the $4.5-7.5 \mathrm{keV}$ energy band. We have defined and computed in an automatic way a Background Estimator Parameter (see Appendix B) which is roughly proportional

\footnotetext{
${ }^{4}$ The good-time interval is defined as the on-axis exposure time taken from the exposure map produced in the XMM-Newton pipeline processing system.
}

to the "real background" in the MOS2 images used. The set of fields that have been used to define the HBSS sample must have the Background Estimator Parameter less than 100 .

Note that we have also considered the EPIC MOS2 observations in large- and small-window mode satisfying the criteria discussed above; in these cases we have excluded from the analysis a circular area of 8 arcmin radius enclosing the central chip. No restrictions on the blocking filter in front of the MOS2 camera have been applied since, as shown in Sect. 2.6, the filter used does not affect the statistical properties of the sample ${ }^{5}$.

The complete BSS sample reported here is based on the analysis of 237 XMM-Newton fields, while the complete HBSS sample is based on a "restricted" data set of 211 XMM-Newton pointings.

In Table 2 we report basic information on the XMM-Newton MOS2 fields used for the sample selection; in particular we list the XMM-Newton observation number, the blocking filter in front of the MOS2 instrument, the Right ascension and Declination of the MOS2 image center, the on-axis good-time exposure for the MOS2 detector, the logarithm of the Galactic Hydrogen column density along the line of sight (from Dickey \& Lockman 1990), the inner and outer radius of the part of the MOS2 image used in the survey, and the total number of BSS and HBSS sources found in the surveyed area of each MOS2 image. In Table 2 we have also marked the 26 MOS2 fields not used for the production of the HBSS sample.

In Fig. 1 we show the distribution of the MOS2 on-axis good-time exposure, the distribution of the Galactic hydrogen column density along the line of sight and the distribution of the Background Estimator Parameter for the XMM-Newton MOS2 data-set used.

\subsection{The XMM-Newton BSS and HBSS samples}

Applying the source selection criteria discussed in Sect. 2.4 to the MOS2 fields reported in Table 2 we have selected 400 XMM-Newton sources: 389 sources belong to the BSS sample and 67 sources to the HBSS sample with 56 sources in common. Basic information on the sources are reported in Table 3 (BSS) and in Table 4 (HBSS); in particular we report the source name, the XMM-Newton observation number, Right Ascension and Declination (J2000) of the X-ray source position, the angular distance (in arcmin) between the source and the MOS2 image center, the source count rate in the $0.5-4.5 \mathrm{keV}$ energy band (BSS sample) or in the $4.5-7.5 \mathrm{keV}$ energy band (HBSS sample), the hardness ratios computed as described in Sect. 3.3, and the optical spectroscopic classification (see Sect. 3.2 for details). In Table 4 we have also marked the 11 sources belonging to the HBSS sample but not to the BSS sample.

In Fig. 2 we show the surface density of the sources belonging to the BSS and to the HBSS as a function of: the MOS2

\footnotetext{
5 The fraction of MOS2 images with a thin, medium and thick filter used here are $\sim 48 \%, \sim 46 \%$ and $\sim 6 \%$, respectively.
} 

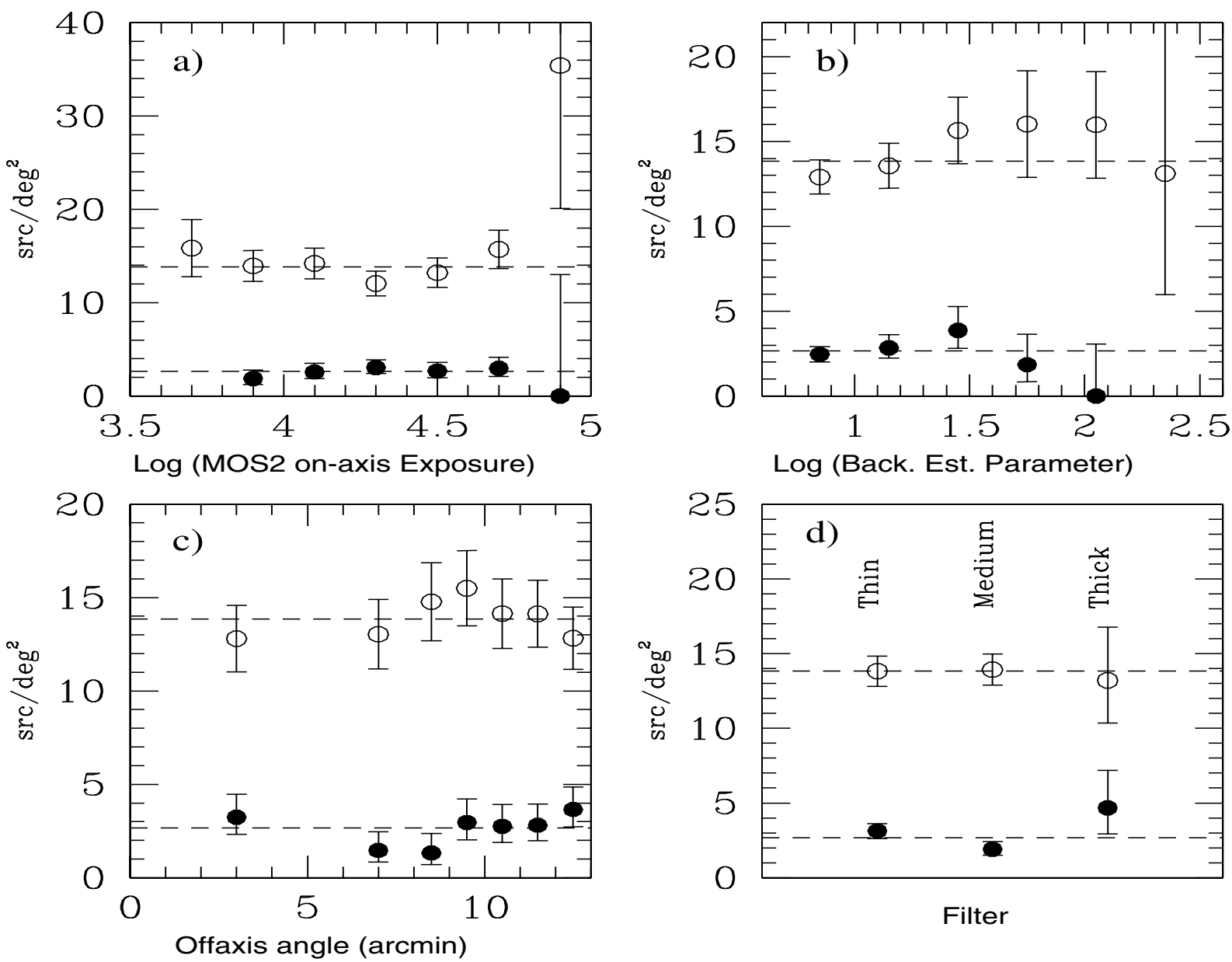

Fig. 2. Panel a): source surface density as a function of the MOS2 on-axis good-time exposure for the sources belonging to the BSS (open circles) and to the HBSS (filled circles) sample. Panel b): source surface density as a function of the Background Estimator Parameter; symbols as in panel a). Panel c): source surface density as a function of the offaxis angle; the bin size has been adapted in order to have similar areas in each bin; symbols as in panel a). Panel d): source surface density as a function of the blocking filter in front of the MOS2 detector; symbols as in panel a). For all the panels the dashed lines correspond to the mean surface density considering the whole BSS or HBSS sample. Errors have been computed using Poisson statistic.

on-axis good-time exposure (panel a); the Background Estimator Parameter (panel b); the off-axis angle (panel c); and the blocking filter in front of the MOS2 detector (panel d). The appropriate area covered in each bin has been considered and errors have been computed using Poisson statistic. The dashed lines reported in Fig. 2 correspond to the mean surface density obtained considering the whole sample. As can be seen there is no significant trend of the source surface density with respect to the plotted parameters confirming a flat sensitivity across the field (i.e. flat sky coverage at the sampled fluxes). The only point which seems to be a factor $\sim 2.5$ above the other is the bin at the highest exposure time in the BSS sample (see panel a). This excess is due to 5 sources found in the field 0022740101 (centered on the Lockman hole), the only pointing in the bin considered; however the error bars are large and so the reported surface density is not significantly different from the mean value. The absolute source surface density as a function of the flux $(\log N-\log S)$ is also in very good agreement with previous and new measurements (see Sect. 3.1) making us confident of the correctness of the data analysis and source selection.

\section{First results}

\subsection{The number-counts relationship(s)}

In Fig. 3 we show (filled circles) a binned representation of the extragalactic ${ }^{6}$ number-flux relationships in the $0.5-4.5 \mathrm{keV}$ energy band (panel a) and in the $4.5-7.5 \mathrm{keV}$ energy band (panel b). As already shown in Sect. 2.6, the sky coverage of this survey at the flux limit used to define the BSS and the HBSS samples is flat and equal to 28.10 sq.deg and 25.17 sq.deg, respectively; given the flat sky coverage the errors in the binned representation are Poissonian errors on the total number of sources having a flux greater than any fixed flux. A conversion factor appropriate for a power-law spectral model with energy index equal to $0.8(0.7)$ in the $0.5-4.5 \mathrm{keV}$ $(4.5-7.5 \mathrm{keV})$ energy band, filtered by an $N_{\mathrm{H}_{\text {Gal }}} \sim 3 \times 10^{20} \mathrm{~cm}^{-2}$

\footnotetext{
${ }^{6}$ Since we are primarily interested in the extragalactic numberflux relationship we have excluded from the computation the sources classified as stars (see Sect. 3.2). Based on the results presented in Sect. 3.5 we are confident that the large majority of the unidentified sources are associated to extragalactic objects.
} 

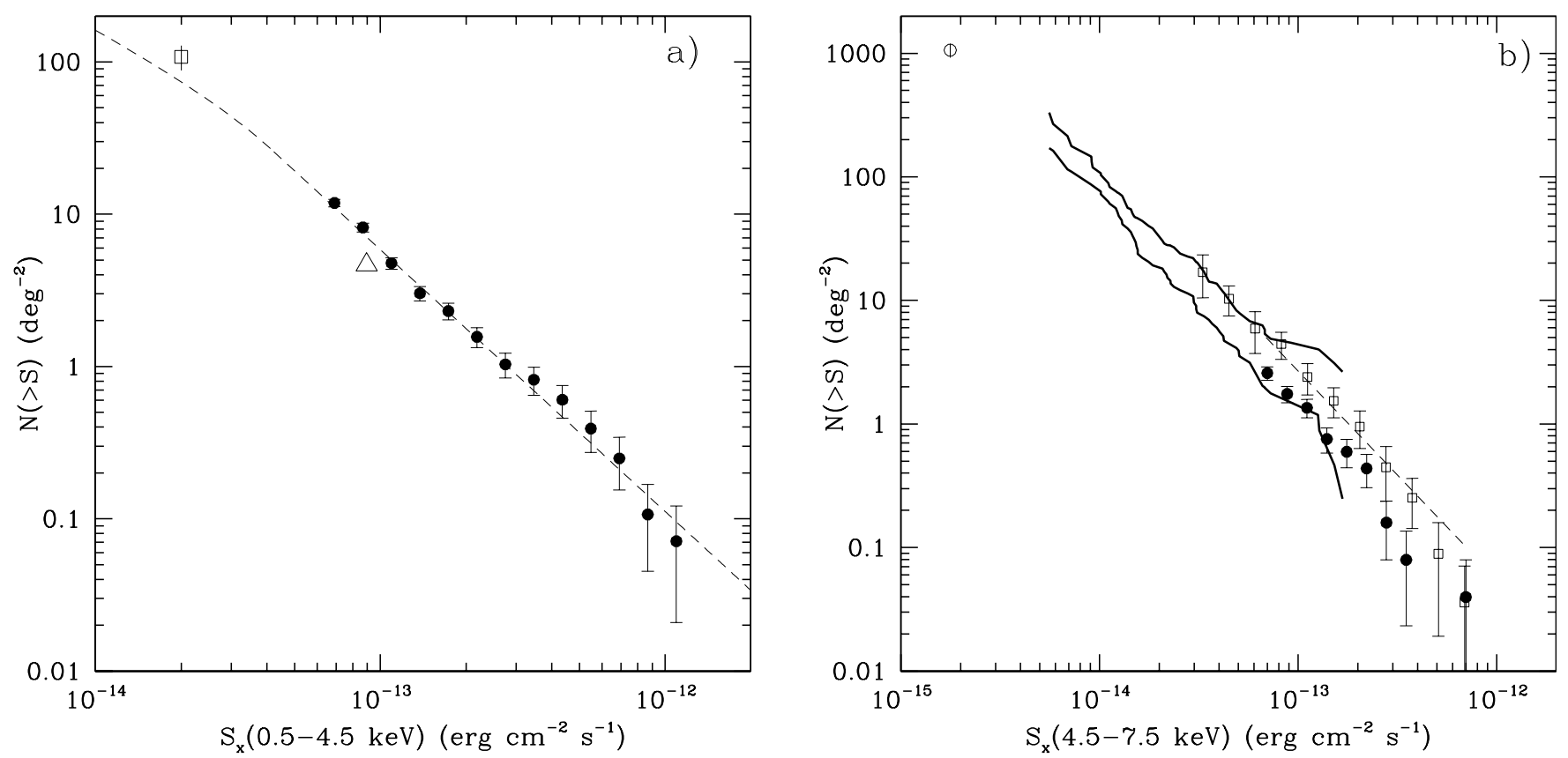

Fig. 3. The extragalactic number-flux relationship in the $0.5-4.5 \mathrm{keV}$ energy band (panel a)) and in the 4.5-7.5 keV energy band (panel b)) obtained using the BSS and HBSS samples (binned representation: black filled circles). In the $0.5-4.5 \operatorname{keV} \log (N>S)-\log S$ (panel a)) we have also reported the ROSAT $(0.5-2.0 \mathrm{keV}) \log (N>S)-\log S$ (dashed line) and the EMSS $(0.3-3.5 \mathrm{keV})$ extragalactic number density at $\sim 10^{-13} \mathrm{erg} \mathrm{cm}^{-2} \mathrm{~s}^{-1}$ (open triangle) both converted to the $0.5-4.5 \mathrm{keV}$ energy band. The open square at $S \simeq 2 \times 10^{-14} \mathrm{erg} \mathrm{cm}^{-2} \mathrm{~s}^{-1} \mathrm{represents}$ the extragalactic surface density $(0.5-4.5 \mathrm{keV})$ obtained by the XMM-Newton AXIS Medium Survey project. In panel b) (4.5-7.5 keV $\log (N>S)-\log S)$ we have also reported the HELLAS2XMM $\log (N>S)-\log S$ interval (area inside the thick solid lines), the HELLAS $\log (N>S)-\log S$ (open squares) and the SHEEP $\log (N>S)-\log S$ (dashed line). The open circle at $S \simeq 2 \times 10^{-15} \mathrm{erg} \mathrm{cm}^{-2} \mathrm{~s}^{-1}$ represents the extragalactic surface density (5-10 keV band) in the Lockman hole field. Both these latter number densities have been converted from their original (5-10 keV) band to the $4.5-7.5 \mathrm{keV}$ band.

(the median value of the $N_{\mathrm{H}_{\mathrm{Gal}}}$ of the survey), has been used in the conversion between the count rate and the flux. The energy spectral index used in the $0.5-4.5 \mathrm{keV}$ energy band corresponds to the "average" one of the extragalactic BSS population in the same energy selection band (see Sect. 3.3). In the 4.5-7.5 keV energy band we have used $\alpha_{\mathrm{E}}=0.7$, the same energy spectral index assumed from other recent surveys in the 5-10 keV band (e.g. the HELLAS2XMM survey, Baldi et al. 2002) and very close to the median energy spectral index in the $0.5-4.5 \mathrm{keV}$ band of the extragalactic HBSS population (see below for details). We recall that, given the median $N_{\mathrm{H}_{\text {Gal }}}$ of the survey, the count rate to flux conversion factor in the $0.5-4.5 \mathrm{keV}(4.5-7.5 \mathrm{keV})$ energy band are accurate $\sim \pm 20$ $(\sim \pm 8) \%$ for an energy spectral index in the range between 0 to 2 .

Both $\log N(>S)-\log S$ distributions can be well described by a power-law model $N(>S) \propto S^{-\alpha}$; their best fit spectral parameters, obtained applying the maximum likelihood method to the unbinned data (see Maccacaro et al. 1982 for details), are reported in Table 5.

The fits have been performed from a flux of $\simeq 7 \times$ $10^{-14} \mathrm{erg} \mathrm{cm}^{-2} \mathrm{~s}^{-1}$ (the faintest flux) to a flux of $\simeq 1 \times$ $10^{-12} \mathrm{erg} \mathrm{cm}^{-2} \mathrm{~s}^{-1}$ (we have excluded from the fit 3 extragalactic BSS sources brighter than this flux limit). For fluxes brighter than this limit we may not be complete since "bright" $\mathrm{X}$-ray sources were chosen as targets of observations and then excluded, by definition, from the survey. However we
Table 5. The extragalactic $\log (N>S)-\log S$ maximum likelihood best fit parameters: $N(>S)=K \times\left(S / 10^{-13}\right)^{-\alpha}$, where $N(>S)$ is the surface densities of sources having a flux greater than $S$ in $\operatorname{deg}^{-2}$.

\begin{tabular}{lrll}
\hline \hline Sample & Objects & $\alpha$ & $K$ \\
& & & \\
$(1)$ & $(2)$ & $(3)$ & $(4)$ \\
\hline $\mathrm{BSS}^{a}$ & 330 & $1.80_{1.69}^{1.91}$ & $6.1_{6.4}^{5.8}$ \\
HBSS & 65 & $1.64_{1.41}^{1.89}$ & $1.5_{1.6}^{1.4}$ \\
HBSS AGN1 & 41 & $1.72_{1.42}^{2.03}$ & $0.92_{1.0}^{0.82}$ \\
HBSS AGN2 & 21 & $1.57_{1.18}^{1.99}$ & $0.50_{0.58}^{0.42}$ \\
\hline
\end{tabular}

Columns are as follows: (1) Sample; (2) Number of sources used in the fit; (3) Best fit power-law slope and 68\% confidence intervals; (4) $\log (N>S)-\log S$ normalization and $68 \%$ confidence intervals. Note that the normalization $K$ is not a parameter of the fit but is determined by re-scaling the model to the number of objects in the sample. The normalization $K$ reported here corresponds to the surface densities at $1 \times 10^{-13} \mathrm{erg} \mathrm{cm}^{-2} \mathrm{~s}^{-1}$. NOTE $-{ }^{a}$ As explained in Sect. 3.1 we have excluded from the fit the 3 BSS extragalactic sources having a flux greater than $1 \times 10^{-12} \mathrm{erg} \mathrm{cm}^{-2} \mathrm{~s}^{-1}$.

note that the surface density of sources with flux greater than $\sim 1 \times 10^{-12} \mathrm{erg} \mathrm{cm}^{-2} \mathrm{~s}^{-1}$ is such that about $2.7(0.9)$ sources in the BSS (HBSS) sample are expected given the covered sky area; these numbers are fully consistent with what is observed. 
Both $\log (N>S)-\log S$ derived here have been compared with a number of representative $\log (N>S)-\log S$ reported in the literature. In particular, in Fig. 3 panel a, we have reported: a) the extragalactic ROSAT $(0.5-2.0 \mathrm{keV})$ $\log (N>S)-\log S$ from Hasinger et al. (1998) (dashed line); b) the EMSS (0.3-3.5 keV) extragalactic number density at $\sim 10^{-13} \mathrm{erg} \mathrm{cm}^{-2} \mathrm{~s}^{-1}$ (open triangle; Gioia et al. 1990); and c) the extragalactic surface density obtained from the XMMNewton AXIS Medium Survey team in the 0.5-4.5 keV energy range (Barcons et al. 2002; open square at $S \simeq 2 \times$ $10^{-14} \mathrm{erg} \mathrm{cm}^{-2} \mathrm{~s}^{-1}$ ). To convert the ROSAT $0.5-2.0 \mathrm{keV}$ band fluxes and the EMSS $0.3-3.5 \mathrm{keV}$ band fluxes into 0.5-4.5 keV fluxes we have used a power-law spectral model having $\alpha_{\mathrm{E}} \simeq 1.0$, corresponding to the mean spectral index of the ROSAT and the EMSS sources (see Hasinger et al. 1993 and Maccacaro et al. 1988, respectively).

In panel b) we have compared our result with: a) the extragalactic XMM-Newton $(5-10 \mathrm{keV}) \log (N>S)-\log S$ from the HELLAS2XMM survey (area inside the thick solid lines; Baldi et al. 2002); b) the BeppoSAX-HELLAS (5-10 keV) $\log (N>S)-\log S$ (open squares; Fiore et al. 2001); c) the ASCA-SHEEP $(5-10 \mathrm{keV}) \log (N>S)-\log S$ (dashed line; Nandra et al. 2003). Finally, the open circle at $S \simeq$ $2 \times 10^{-15} \mathrm{erg} \mathrm{cm}^{-2} \mathrm{~s}^{-1}$ represents the extragalactic surface density in the (5-10 keV) energy band from the XMM-Newton observation of the Lockman hole field (Hasinger et al. 2001). For consistency with previous hard survey both these latter number densities have been converted from their original $(5-10 \mathrm{keV})$ band to the $4.5-7.5 \mathrm{keV}$ band using a power-law spectral model having $\alpha_{\mathrm{E}}=0.7$. We found that our results are fully consistent with those obtained from the other XMM-Newton related survey (e.g. the HELLAS2XMM 5-10 keV survey) and, moreover, our better statistics above $7 \times 10^{-14} \mathrm{erg} \mathrm{cm}^{-2} \mathrm{~s}^{-1}$ allow us to significantly constrain the $4.5-7.5 \mathrm{keV}$ extragalactic number densities above this flux.

On the other hand the extragalactic $\operatorname{HBSS} \log (N>$ $S$ ) $-\log S$ falls below both the BeppoSAX-HELLAS and the ASCA-SHEEP determinations. Given the results discussed in Sect. 2.6 and the very similar slope between our $\log (N>$ $S)-\log S$ and the BeppoSAX/ASCA $\log (N>S)-$ $\log S$ we have checked if this problem could be related to an offset of the absolute flux scale in the $4.5-7.5 \mathrm{keV}$ energy range between XMM-Newton and BeppoSAX/ASCA. To this purpose we have cross-correlated the HELLAS and the SHEEP sources with the total catalogue of XMM-Newton sources obtained from the analysis of the 237 XMM-Newton fields reported in Table 2. Using a search radius of 90" and considering the point-like XMM-Newton sources with a 4.5-7.5 keV likelihood parameter greater than 12 and with an "Illumination Factor" (see Appendix A for details) greater than 0.8 , we have found 6 "bona fide" HELLAS-XMM coincidences and 2 "bona fide" SHEEP-XMM coincidences. The ratio between the 4.5-7.5 keV XMM-Newton fluxes and the 5-10 keV BeppoSAX fluxes in the case of the HELLAS sources ranges between 0.09 and 0.98 with a mean value of 0.47 , while in the case of the two SHEEP sources the ratio between the 4.5-7.5 keV XMM-Newton fluxes and the $5-10 \mathrm{keV}$ ASCA fluxes is equal to $\simeq 0.63$ for both objects.
Table 6. The current optical breakdown of the BSS and HBSS samples.

\begin{tabular}{lrr}
\hline \hline & BSS $^{1}$ & HBSS \\
\hline Objects $^{2}$ & $389(166)$ & 67 \\
& & \\
Identified: & $278(146)$ & 60 \\
Identification rate & $71 \%(88 \%)$ & $90 \%$ \\
& & \\
AGN-1 & $180(100)$ & 39 \\
AGN-2 & $26(15)$ & 16 \\
Galaxies & $39(3)$ & 1 \\
Clusters of Galaxies & $4(1)$ & 1 \\
BL Lacs & $5(3)$ & 1 \\
Stars $^{4}$ & $56(24)$ & 2 \\
\hline
\end{tabular}

1 In brackets we have reported the optical breakdown for the BSS sources with Right Ascension below $5^{\mathrm{h}}$ or above $17^{\mathrm{h}} ;{ }^{2}$ Note that 56 sources are in common between the BSS and HBSS samples; ${ }^{3}$ We stress that some of the sources classified as "Optical Normal Galaxy" could indeed host an optically elusive AGN (see e.g. Severgnini et al. 2003); ${ }^{4}$ To our knowledge all but one (XBS J014100.6-675328) of the sources classified as stars are coronal emitters.

Although these small numbers do not allow us to draw firm conclusions we note that using a conversion factor between the fluxes in the 5-10 keV energy range and the fluxes in the $4.5-7.5 \mathrm{keV}$ energy range equal to 0.47 (instead of 0.69 as expected for $\alpha_{\mathrm{E}}=0.7$ and as assumed in Fig. 3) the HBSS $\log (N>S)-\log S$ and the BeppoSAX-HELLAS $\log (N>$ $S)-\log S$ turn out to be in perfect agreement. This suggests that an offset in the absolute flux scale could easily explain the disagreement in the number densities discussed above; this possible discrepancy in the flux scale has to be further investigated.

\subsection{Optical identification and classification}

One of the main characteristics of the X-ray sources presented here is that the majority ( $90 \%$ ) of them have an optical counterpart above the POSS II limit $\left(R \sim 21^{\mathrm{mag}}\right)$, thus allowing spectroscopic identification even on 2-4 meter class telescopes. Furthermore, given the good accuracy of the X-ray positions $^{7}$ and the magnitude of the optical counterparts there is no ambiguity in the optical identification for the large majority of cases.

Up to now $285 \mathrm{X}$-ray sources have been spectroscopically identified (either from the literature or from our own observations mainly at the Italian "Telescopio Nazionale Galileo" -TNG, at the ESO $3.6 \mathrm{~m}$, at the Calar Alto $2.2 \mathrm{~m}$ or at

\footnotetext{
7 Using the optical position of the sources classified as type 1 AGN we have evaluated that the $90 \%$ confidence level error circle has a radius equal to $\sim 4^{\prime \prime}$; about $99 \%$ of the type 1 AGN are within $6^{\prime \prime}$ from the X-ray position. This is consistent with what found in other XMMNewton surveys (Barcons et al. 2002; Fiore et al. 2003).
} 
the NOT $2.6 \mathrm{~m}^{8}$ telescopes) leading to a $71 \%$ and $90 \%$ identification rate for the BSS and HBSS samples respectively.

The optical breakdown of the sources identified so far is reported in Table 6 . We stress that the source detection algorithm is optimized for point-like sources, so the sample of clusters of galaxies is not statistically complete nor representative of the cluster population.

To our present knowledge all but one (XBS J014100.6-6753289) of the sources classified as stars are coronal emitters. If we consider the BSS sources with Right Ascension below $5^{\mathrm{h}}$ or above $17^{\mathrm{h}}$ (spectroscopic identification rate of $\sim 88 \%$, see Table 6 ), the X-ray coronal emitting stars represent $\sim 14 \%$ of the $|b|>20^{\circ}(0.5-4.5 \mathrm{keV})$ population at the sampled fluxes. This fraction must be compared with $\sim 1.5 \%$ of coronal emitters in the HBSS sample ${ }^{10}$; this smaller fraction of stars in the HBSS sample, compared with that in the BSS sample, is entirely consistent with their low temperature coronal emission. Note that in the softer $(0.5-2.0 \mathrm{keV})$ ROSAT Bright Survey Catalog (RBS, Schwope et al. 2000) the fraction of coronal emitting stars is around $37 \%$.

The large majority ( $90 \%)$ of the extragalactic X-ray sources are emission line objects, i.e. sources for which at least one strong ( $E W \gg 5 \AA$ in the source rest frame) emission line is present in the optical spectrum. As a comparison in the RBS the fraction of emission line AGN amongst the extragalactic sources is around 55\% (Schwope et al. 2000). The few remaining non-emission line objects have been classified as "Normal Galaxies" or BL Lacs objects according to the measured Calcium break discontinuity at the rest frame wavelength of $4000 \AA$ (see e.g. Landt et al. 2002). We stress that some of the sources classified as "Normal Galaxies" could indeed host an AGN. As already discussed by Severgnini et al. (2003) using $\mathrm{X}$-ray and optical spectral data from this project, the lack of significant emission lines in the optical spectra can be explained by an adequate combination of the absorption associated with the AGN and of the optical faintness of the active nucleus with respect to the host galaxy. Furthermore for some of the sources classified as "Normal Galaxies" the $\mathrm{H}_{\alpha}$ line (in some case the only spectroscopic evidence of the presence of an AGN in the optical domain) is not sampled. Although the presence of an AGN in the nucleus of some of these sources is highly probable (e.g. observed $L_{x}$ well in excess of $10^{42} \mathrm{erg} \mathrm{s}^{-1}$ ) we prefer to wait for a confirmation also from optical/infrared followups; for the moment these objects are classified as "Normal Galaxies".

\footnotetext{
${ }^{8}$ As part of the AXIS (An XMM-Newton International Survey) project, see http://www.ifca.unican.es/ xray/AXIS

9 XBS J014100.6-675328 (BL Hyi), also belonging to the HBSS sample, is a well known AM Herculis object (a polar) i.e. a binary system composed of a magnetic white dwarf and a low-mass star (see Caccianiga et al. 2004, and references therein).

${ }^{10}$ On the basis of the results presented in Sect. 3.5 we are confident that the bulk of unidentified sources both in the BSS and HBSS samples are associated with extragalactic objects and we know that XBS J014100.6-675328 is an accreting binary system (see Caccianiga et al. 2004).
}

To classify the emission line objects we have used the criteria presented for instance in Veron-Cetty \& Veron (2001) which are based on the line width and the line flux ratios. Type 1 AGN are those sources showing broad $\left(F W H M>1000 \mathrm{~km} \mathrm{~s}^{-1}\right)$ permitted lines, while type $2 \mathrm{AGN}$ are those sources showing only narrow lines $\left(F W H M<1000 \mathrm{~km} \mathrm{~s}^{-1}\right)$ and, when detected, $[\mathrm{OIII}] \lambda 5007 / \mathrm{H}_{\beta}>3$.

A few sources show permitted lines with $1000 \mathrm{~km} \mathrm{~s}^{-1}<$ $F W H M<2000 \mathrm{~km} \mathrm{~s}^{-1}$ and $[\mathrm{OIII}] \lambda 5007 / \mathrm{H}_{\beta}$ below 3. These sources are probably narrow line Seyfert 1 candidates and, according to our classification, have been included in the type 1 AGN group. For some sources classified as type 2 AGN we have indication of the presence of a broad component at the bottom of the narrow $\mathrm{H}_{\beta}$ and/or $\mathrm{H}_{\alpha}$ lines. These sources should be properly classified as Seyfert 1.8 or Seyfert 1.9 objects; for the purpose of the present paper these sources have been included in type 2 AGN group. Finally for 26 objects a better $S / N$ optical spectrum and/or a more appropriate set-up for the spectroscopic observations are needed to firmly classify them as type 1 or type 2 AGN. These 26 sources have been marked in Col. 8 of Tables 3 and 4 .

\section{3. $0.5-4.5 \mathrm{keV}$ spectral properties}

A "complete" spectral analysis for all the sources in the BSS and HBSS samples (using data from the two EPIC MOSs and the EPIC pn) is in progress; first results on selected subsamples of sources have been already discussed in Severgnini et al. (2003) and Caccianiga et al. (2004). In the meantime, and in order to extract first order X-ray spectral information we present here a "Hardness Ratio" analysis of the single sources using only EPIC MOS2 data; this latter method is equivalent to the "color-color" analysis largely used at optical wavelengths. The use here of the "Hardness Ratio" analysis is twofold. First of all it is much faster than a complete spectral analysis with the combined use of three different instruments. Second, a "Hardness Ratio" is often the only X-ray spectral information available for the faintest sources in the XMM-Newton catalogue, and thus, a "calibration" in the parameter space is needed to select "clean" and well-defined samples. On the other hand, in Caccianiga et al. (2004) we have already shown and discussed a tight correlation between X-ray absorption, as deduced from a complete X-ray spectral analysis, and "Hardness Ratio" properties.

We have used the hardness ratios as defined from the XMM-Newton pipeline processing ${ }^{11}$ :

$$
H R 2=\frac{C(2-4.5 \mathrm{keV})-C(0.5-2 \mathrm{keV})}{C(2-4.5 \mathrm{keV})+C(0.5-2 \mathrm{keV})}
$$

\footnotetext{
11 We have not used here the "pipeline processing product" HR1 which is defined using the corrected count rate in the $(0.15-0.5) \mathrm{keV}$ and in the (0.5-2) keV energy band since the measured count rate in the $(0.15-0.5) \mathrm{keV}$ band is a strong function of the Galactic $N_{\mathrm{H}}$ along the line of sight. Note that the effect on $H R 2$ and $H R 3$ due to the different $N_{\mathrm{H}_{\mathrm{Gal}}}$ for the objects in the sample (which ranges between $\sim 10^{20}$ to $10^{21} \mathrm{~cm}^{-2}$ ) is negligible.
} 

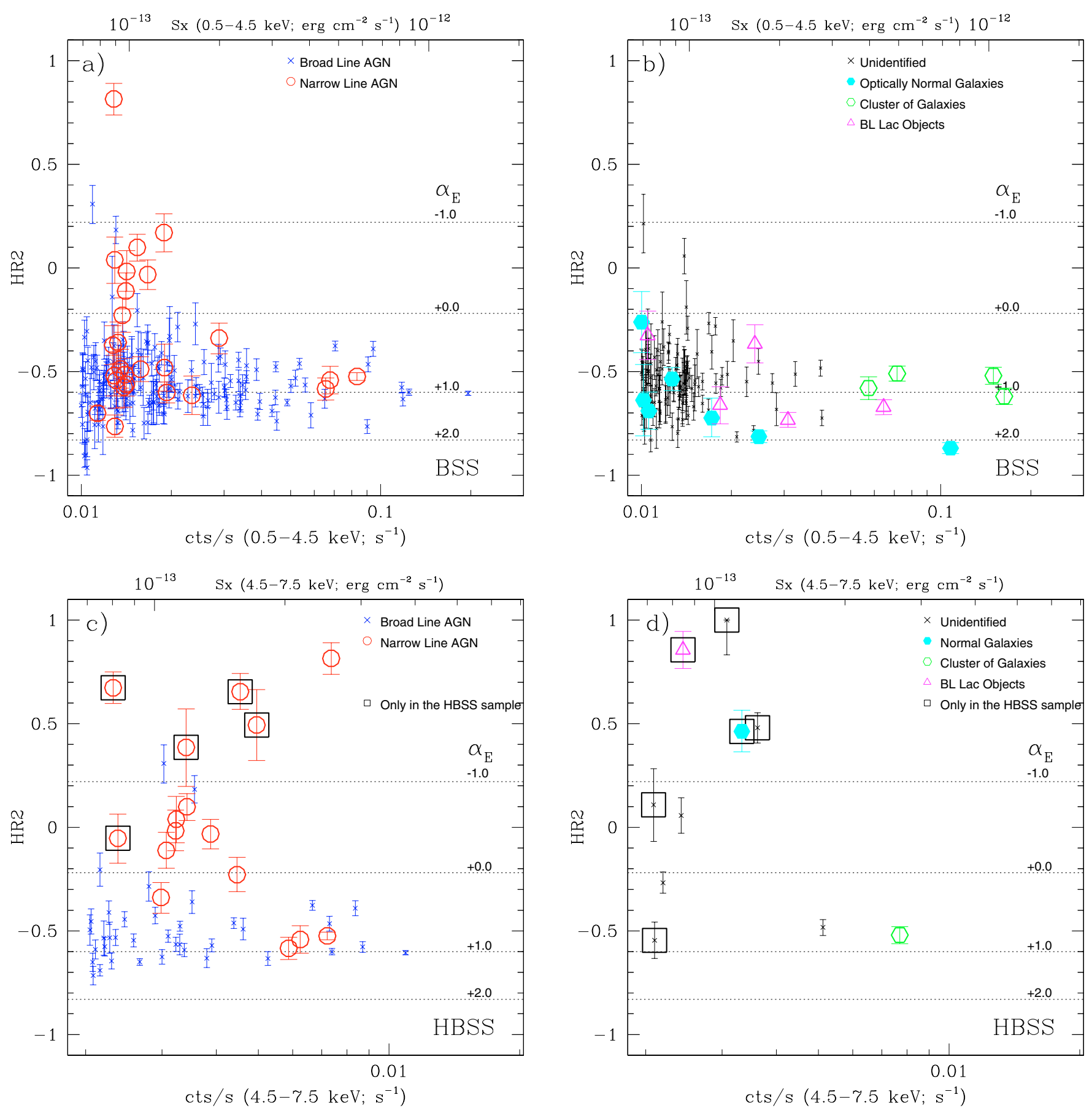

Fig. 4. $H R 2$ vs. EPIC MOS 2 count rate in the selection band for the sources in the BSS (panel a) and panel b)) and HBSS sample (panel c) and panel d)). We have also reported the $H R 2$ expected from a unabsorbed power-law model with $\alpha_{\mathrm{E}}$ ranging from -1 to $2\left(S_{\mathrm{E}} \propto E^{-\alpha_{\mathrm{E}}}\right)$. The flux scale on the top has been computed assuming a conversion factor appropriate for $\alpha_{\mathrm{E}} \sim 0.8$ (BSS sample) or $\alpha_{\mathrm{E}} \sim 0.7$ (HBSS sample). We have used different symbols to mark the different kinds of objects. The eleven sources belonging only to the HBSS sample are enclosed inside empty squares in panels c) and d).

and

$H R 3=\frac{C(4.5-7.5 \mathrm{keV})-C(2-4.5 \mathrm{keV})}{C(4.5-7.5 \mathrm{keV})+C(2-4.5 \mathrm{keV})}$

where $\mathrm{C}(0.5-2 \mathrm{keV}), \mathrm{C}(2-4.5 \mathrm{keV})$ and $\mathrm{C}(4.5-7.5 \mathrm{keV})$ are the "PSF and vignetting corrected" count rates in the $0.5-2$, 2-4.5 and 4.5-7.5 keV energy bands, respectively.
In Fig. 4 (panel a and panel b) we plot $H R 2$ as a function of the $0.5-4.5 \mathrm{keV}$ count rate for the extragalactic (and unidentified) sources of the BSS sample. In particular, in panel a) we show the position of the optically classified type 1 and type 2 AGN, while in panel b) we have shown the unidentified sources, the "Optically Normal Galaxies", the Clusters of galaxies and the BL Lac objects. On the top, we have also reported the flux scale computed assuming a conversion factor 
Table 7. HR2 statistic for some relevant BSS and HBSS sub-sample(s).

\begin{tabular}{lrrrrrr}
\hline \hline $\begin{array}{l}\text { Sample } \\
(1)\end{array}$ & $\begin{array}{r}\text { Objects } \\
(2)\end{array}$ & $\begin{array}{r}\text { Weighted } \\
(3)\end{array}$ & $\begin{array}{r}\text { Unweighted } \\
(4)\end{array}$ & $\begin{array}{r}\text { Median } \\
(5)\end{array}$ & $\begin{array}{r}\text { St.Dev. } \\
(6)\end{array}$ & $\begin{array}{r}\sigma \text { intrins. } \\
(7)\end{array}$ \\
\hline BSS Sample: & & & & & & \\
Extragalactic & 333 & $-0.51 \pm 0.01$ & $-0.54 \pm 0.01$ & -0.57 & 0.20 & 0.16 \\
ÃGN1 & 180 & $-0.54 \pm 0.01$ & $-0.56 \pm 0.01$ & -0.58 & 0.15 & 0.12 \\
ÃGN2 & 26 & $-0.32 \pm 0.07$ & $-0.34 \pm 0.07$ & -0.49 & 0.35 & 0.32 \\
$\quad$ Unidentified & 111 & $-0.52 \pm 0.02$ & $-0.54 \pm 0.02$ & -0.56 & 0.16 & 0.12 \\
Stars & 56 & $-0.83 \pm 0.01$ & $-0.88 \pm 0.01$ & -0.90 & 0.11 & 0.08 \\
HBSS Sample: & & & & & & \\
Extragalactic & 65 & $-0.10 \pm 0.05$ & $-0.27 \pm 0.05$ & -0.47 & 0.44 & 0.40 \\
ÃGN1 & 39 & $-0.44 \pm 0.03$ & $-0.49 \pm 0.03$ & -0.53 & 0.21 & 0.18 \\
ÂGN2 & 16 & $0.14 \pm 0.11$ & $0.05 \pm 0.11$ & -0.02 & 0.45 & 0.44 \\
\hline
\end{tabular}

Columns are as follows: (1) Sample; (2) Number of sources; (3) $H R 2$ weighted average; (4) $H R 2$ unweighted average; (5) $H R 2$ median value; (6) Observed standard deviation of the HR2 distribution; (7) Intrinsic dispersion of the $H R 2$ distribution computed using the maximum likelihood method as described in Maccacaro et al. (1988).

appropriate for $\alpha_{\mathrm{E}} \simeq 0.8$, which is the "mean" energy spectral index of the "extragalactic" sample in the $0.5-4.5 \mathrm{keV}$ energy band (see below).

No obvious trend in the source spectra as a function of the count rate is measured. If we split the extragalactic BSS sample in two different bins of count rate (below or above a count rate of $1.41 \times 10^{-2} \mathrm{cts} / \mathrm{s}$ in the $0.5-4.5 \mathrm{keV}$ energy band) which includes a similar number of objects (163 and 170 sources, respectively) we found that the $H R 2$ distributions of the two sub-samples are consistent with being extracted from the same distribution with a probability of $61 \%$ according to a KS test.

The weighted average of the extragalactic population is $H R 2=-0.51 \pm 0.01$ corresponding to $\alpha_{\mathrm{E}}=0.74 \pm 0.03$ (the relation between the spectral index and $H R 2$ has been tabulated for few representative spectral indices in Table 1). For comparison the weighted averages of the sources classified as type $1 \mathrm{AGN}$ and unidentified objects are $H R 2=-0.54 \pm 0.01$ and $H R 2=-0.52 \pm 0.02$, respectively. The $H R 2$ distribution of the sources identified as type 1 AGN appears to be "narrow" with $\sim 90 \%$ of the sources inside the $H R 2$ range -0.75 to -0.35 . On the contrary the type 2 AGN are characterized by a broader distribution with $\sim 42 \%$ of the objects having an observed energy spectral index apparently flatter than that of the cosmic $\mathrm{X}$-ray background ( $\alpha_{\mathrm{E}}=0.4$ corresponding to $H R 2=-0.38$ ). For comparison only $\sim 10 \%$ of the unidentified X-ray sources or of the X-ray sources identified as broad line AGN seem to have spectra apparently flatter than $\alpha_{\mathrm{E}}=0.4$.

Please note that two broad line AGN, which are clearly separated from the majority of the other broad line AGN, seem to be characterized by an extremely hard spectrum $\left(\alpha_{\mathrm{E}} \sim-1\right)$. These two objects, belonging both to the BSS and HBSS samples, are XBS J091828.4+513931 $(H R 2=0.31)$ and XBS J143835.1+642928 $(H R 2=0.18)$. For the first object the optical spectra show broad $\mathrm{H}_{\beta}$ and $\mathrm{H}_{\alpha}$ emission lines without any obvious sign of peculiarity, while in the case of XBS J143835.1+642928 the optical spectrum in hand is very noisy and therefore the optical classification is at the moment tentative. The X-ray spectra of both sources are described by an absorbed power-law model having an intrinsic $N_{\mathrm{H}}$ in excess of $10^{22} \mathrm{~cm}^{-2}$. At the moment these two sources are the only broad line AGN displaying intrinsic absorption (as derived from a complete X-ray spectral analysis) above $10^{22} \mathrm{~cm}^{-2}$ but the completion of the X-ray spectral work for the total sample is needed to evaluate correctly the fraction of X-ray absorbed broad line AGN in this survey ${ }^{12}$.

It is now worth comparing the $H R 2$ properties of the extragalactic BSS and HBSS sources; in Fig. 4 (panel c and d) we plot $H R 2$ as a function of the $4.5-7.5 \mathrm{keV}$ count rate for the extragalactic (and unidentified) sources of the HBSS sample. As a class, the type $1 \mathrm{AGN}$ in the HBSS sample seem to have the same $H R 2$, and thus $0.5-4.5 \mathrm{keV}$ spectral properties, of the type $1 \mathrm{AGN}$ in the BSS; about $87 \%$ of them reside in the $H R 2$ range between -0.75 and -0.35 , with a median $H R 2$ value of -0.53 . On the contrary the type 2 AGN in the HBSS seem to be characterized by more extreme spectral properties if compared with the type $2 \mathrm{AGN}$ in the BSS sample; $~ 81 \%$ of them seem to have an energy spectral index flatter than 0.4 and $\sim 2 / 3$ seem to have inverted spectra $\left(\alpha_{\mathrm{E}}<0\right)$. It is worth noting that 2 of the 3 type 2 AGNs having $H R 2$ around $\sim-0.55$ in the HBSS sample are Seyfert 1.9 galaxies.

The HR2 statistic for some relevant BSS and HBSS subsample(s) have been summarized in Table 7.

We note that in the case of the extragalactic population in the BSS sample the use of the weighted average, the unweighted average or the median value of $H R 2$ give consistent results on the underlying spectral index $\left(\alpha_{\mathrm{E}} \sim 0.74, \alpha_{\mathrm{E}} \sim 0.83\right.$ and $\alpha_{\mathrm{E}} \sim 0.91$, respectively). On the contrary in the case of

\footnotetext{
12 We note that in the HBS28 sample discussed in Caccianiga et al. (2004) none of the 19 type 1 AGN is absorbed with $N_{\mathrm{H}}$ in excess to $10^{22} \mathrm{~cm}^{-2}$, implying that the fraction of X-ray absorbed type 1 AGN is less than $10 \%$ of the total type 1 AGN population. This result is also confirmed by the present HBSS sample since at most 4 (out of 39) broad line AGN are characterized by $H R 2$ values typical of absorbed $\left(N_{\mathrm{H}}>10^{22} \mathrm{~cm}^{-2}\right)$ AGN. See also Sect. 3.4.
} 
the extragalactic population in the HBSS sample the use of the weighted average, the unweighted average or the median value of $H R 2$ gives completely different results $\left(\alpha_{\mathrm{E}} \sim-0.27\right.$, $\alpha_{\mathrm{E}} \sim 0.11$ and $\alpha_{\mathrm{E}} \sim 0.64$, respectively). Since the $H R 2$ distribution of the extragalactic population in the HBSS sample is significantly different from a Gaussian we prefer to use as "average" spectral index of this sample that related to the median value $\left(\alpha_{\mathrm{E}} \sim 0.64\right)$.

A similar result has been pointed out by Nandra et al. (2003) studying the spectral properties of the sources in the SHEEP (5-10 keV) survey. These authors prefer the use of the unweighted average over the weighted average (they did not consider median values) and reach the result that $\left\langle\alpha_{\mathrm{E}}\right\rangle \sim 0$ and the conclusion that the 5-10 keV surveys are sampling a completely different population compared with the $2-10 \mathrm{keV}$ surveys. On the contrary we find strong evidence that the $0.5-4.5$ spectral properties of the class of broad line AGN in the BSS and in the HBSS are very similar. Moreover, since the majority of the objects in the BSS and HBSS samples are in common we do not find compelling evidence that the surveys in the two bands are selecting completely different populations. The HBSS survey is simply more efficient than the BSS survey in selecting the hard part of the intrinsic source spectral distribution.

The eleven objects belonging only to the HBSS sample (enclosed inside empty squares in Figs. 4-6) are amongst the hardest X-ray sources in the sample; all but one (XBS J140113.4+024016, still optically unidentified) of them seem to be characterized by an apparently inverted spectrum $\left(\alpha_{\mathrm{E}}<0.0\right)$. Among these sources there are 5 type 2 AGN, one optically normal galaxy, one BLLac object and 4 unidentified objects.

\subsection{Broad-band X-ray spectral properties}

Combining the information on $H R 2$ and $H R 3$ we can now investigate in more detail the broad band spectral properties of the sample(s) as well as the selection function(s) of the BSS and HBSS.

The comparison is made in Fig. 5 where we show the position of the BSS (panels a, b and c) and HBSS (panel d) sources in the $H R 2$ - HR3 plane. We have used different symbols and panels to mark the spectroscopically identified and unidentified objects. Useful information can be extracted by cross-comparing the position of the different optical types of sources as well as by comparing the position of the sources in the BSS and HBSS samples.

The "bulk" of the sources optically identified as broad line AGNs are strongly clustered in the region between $H R 2=-0.75$ and $H R 2=-0.35$; this is true both for the broad line AGNs belonging to the BSS sample and for those belonging to the HBSS sample (only 5 of 39 HBSS broad line AGN are outside these limits). The spread on $H R 3$ is much larger than the spread on $H R 2$ but note that $H R 3$ is much noisier than $H R 2$ since many of the sources are detected with poor statistics (or even undetected) in the $4.5-7.5 \mathrm{keV}$ energy band.
All but $2(\sim 96 \%)$ of the sources classified as stars ${ }^{13}$ in the BSS sample have an $H R 2$ less than -0.75 . If we assume a simple Raymond-Smith thermal model, $H R 2 \leq-0.75$ corresponds to temperatures below $\sim 1.5 \mathrm{keV}$, in very good agreement with the identification as coronal emitting stars.

In Caccianiga et al. (2004), using a "pilot" 4.5-7.5 keV sample composed of $28 \mathrm{X}$-ray sources (26 of which in common with the current version of the HBSS sample reported in Table 4), we have already discussed the correlation between $\mathrm{X}$-ray absorption, as deduced from a complete X-ray spectral analysis, and "Hardness Ratio" properties. In particular we have found that a) at the sampled fluxes, the $4.5-7.5 \mathrm{keV}$ selection is picking up AGN having an intrinsic $N_{\mathrm{H}}$ up to few times $10^{23} \mathrm{~cm}^{-2}$; b) all the AGN having $H R 2>-0.35$ are X-ray absorbed with $N_{\mathrm{H}}$ ranging from few times $10^{21}$ up to few times $10^{23} \mathrm{~cm}^{-2}$. Assuming that this result is valid also for the sources presented here we can conjecture that all the sources having $H R 2$ greater than -0.35 are absorbed AGN; in this part of the diagram, besides narrow line AGN, we also note a few broad line AGN and 2 sources (one in the BSS sample and one in the HBSS sample) optically identified as normal galaxies. However also in these sources their point-like X-ray emission, their X-ray spectra (a preliminary spectral analysis shows that they are described by an absorbed power-law model having $N_{\mathrm{H}}>\sim 10^{22} \mathrm{~cm}^{-2}$ ) and their intrinsic luminosity (in excess of $\sim 7 \times 10^{43} \mathrm{erg} \mathrm{s}^{-1}$ in the $2-10 \mathrm{keV}$ energy range) strongly suggest the presence of an AGN. The existence of relatively luminous X-ray sources, optically identified with "normal galaxies", has been reported since the Einstein Observatory era in the early eighties (Elvis et al. 1981); this kind of sources were called in a variety of names such as optically dull galaxies (Elvis et al. 1981), passive galaxies (Griffiths et al. 1995) and X-ray bright optically normal galaxies (XBONG, Comastri et al. 2002). We have already discussed in Severgnini et al. (2003) that detailed and specific optical-infrared follow-ups or higher-quality optical spectra are needed to unveil the AGN also in the optical domain. An advection-dominated accretion flow model has been recently used by Yuan \& Narayan (2004) to explain their broad band properties.

Contrary to broad line AGNs and stars, narrow line (type 2) AGNs seem to be distributed over a larger area in the $H R 2-H R 3$ plane with a well visible difference in the source position between the type 2 AGNs in BSS and those in the HBSS sample. Although many of them have the hardest spectra amongst the identified objects, highly suggestive of intrinsic absorption (see also Fig. 4), a new fact which seems to emerge from this investigation is the large number of narrow line AGN in the BSS sample occupying the locus typical of Xray unabsorbed broad line AGN. Taking into account the still

\footnotetext{
13 The two sources that are outside the "stellar" $H R 2$ locus are XBS J014100.6-675328 and XBS J215323.7+173018. The first object, XBS J014100.6-675328, has been already discussed in footnote 9. The XMM spectrum, discussed in Caccianiga et al. (2004), is well fitted by a unabsorbed power-law model with $\Gamma \sim 1.53$ plus a thermal component having a temperature $k T \sim 55 \mathrm{eV}$ and an emission line at $E \sim 6.7 \mathrm{keV}$. The second object (XBS J215323.7+173018) is a star having an XMM spectrum described by a thermal component with $k T \sim 1.6 \mathrm{keV}$ and a possible hard tail.
} 

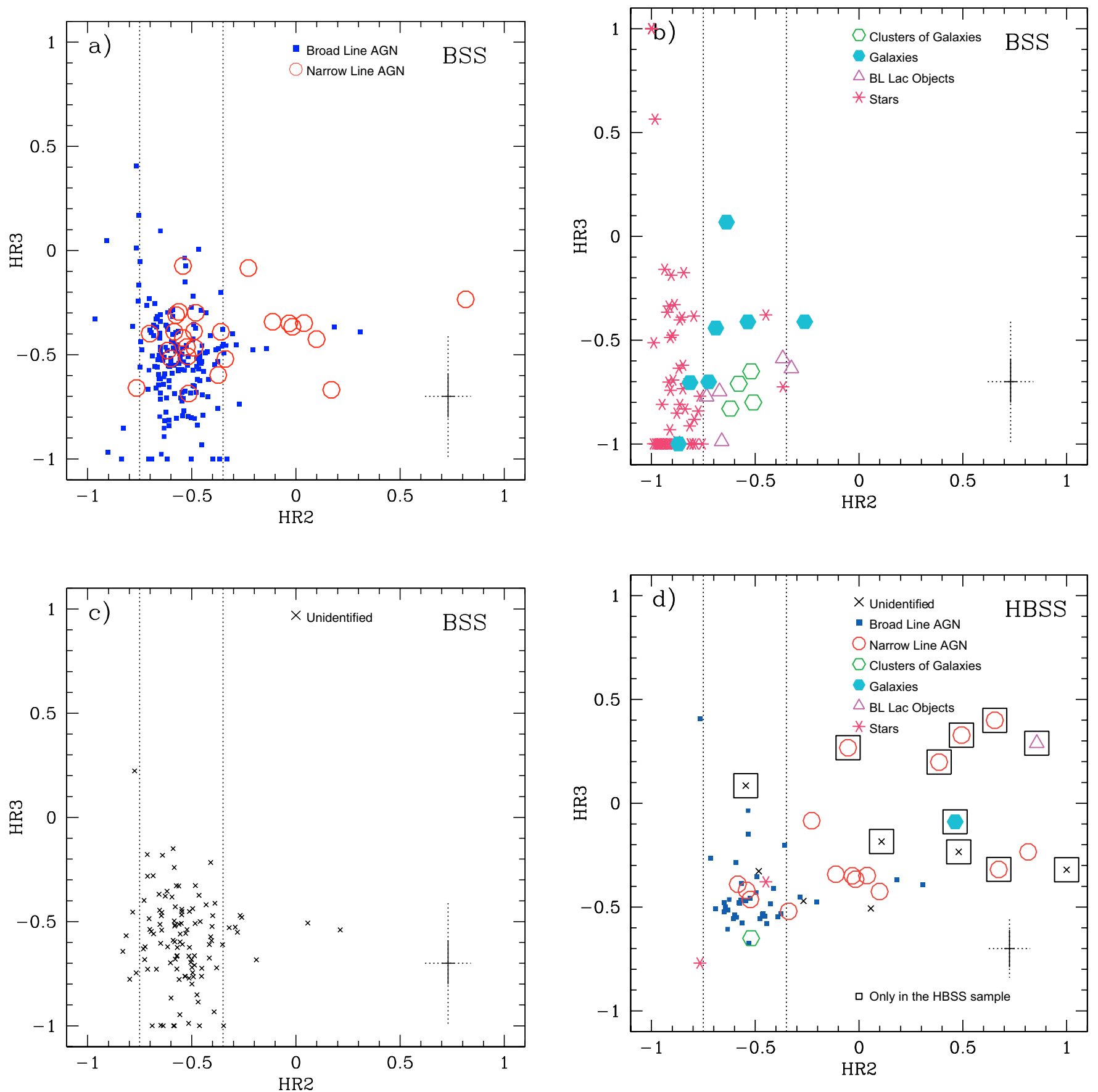

Fig. 5. $H R 2$ vs. $H R 3$ for the sources belonging to the BSS sample (panel a), b) and c)) and to the HBSS sample (panel d)). The dotted lines at constant $H R 2$ correspond to the locus enclosing $~ 90 \%$ of the type 1 AGN in the BSS sample; these lines have been reported in all panels to assist with the comparison(s). We have used different symbols to mark the spectroscopically identified and unidentified objects in the two samples. The eleven sources belonging only to the HBSS sample are enclosed inside empty squares in panel d). In the lower right corner of each panel we have also reported the median error on $H R 2$ and $H R 3$ for the total BSS and HBSS sample (solid line) and the $90 \%$ percentile on these errors (dotted line).

incomplete spectroscopic identification work and that some sources need a better quality optical spectrum we estimate that the relative fraction of these objects over the entire type 2 AGN population may range between $50 \%$ and $75 \%$ in the case of the BSS sample and around $20 \%$ in the case of the HBSS sam$\mathrm{ple}^{14}$. It is also worth noting that 2 out of 4 of the type 2 AGN

\footnotetext{
14 This latter estimate is consistent with the fact that in the HBS28 sample discussed in Caccianiga et al. (2004) there is only one
}

belonging to the (2-10 keV) HELLAS2XMM survey and having a good X-ray statistic (sample S1 in Perola et al. 2004) are characterized by an "observed" intrinsic $N_{\mathrm{H}}$ well below $10^{22} \mathrm{~cm}^{-2}$.

To our knowledge two kinds of narrow line AGN could populate this zone, and thus could have X-ray spectral

type 2 AGN with $H R 2$ between -0.75 and -0.35 out of 8 type 2 objects. 

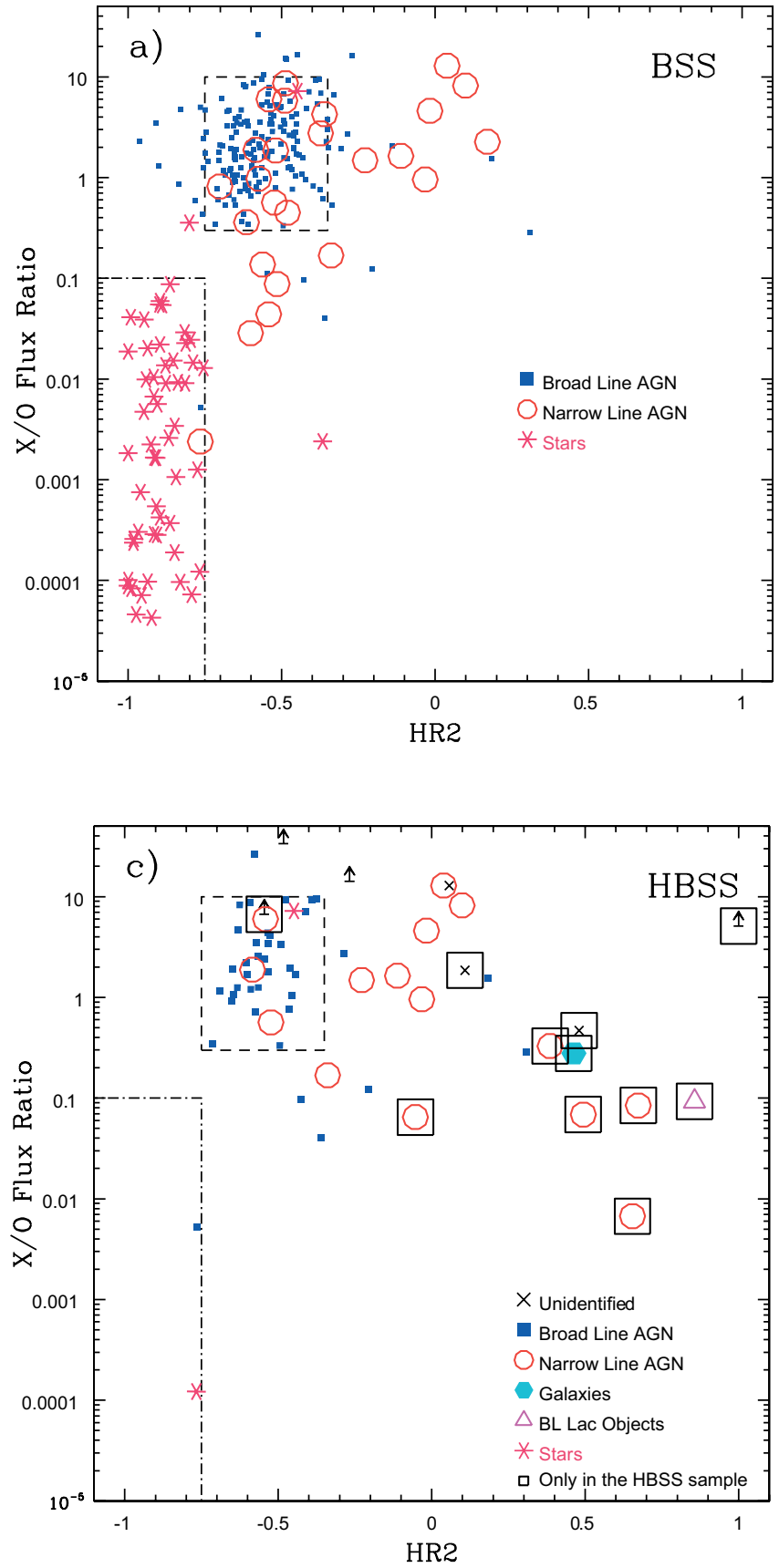

properties similar to those expected from unabsorbed AGN: a) "Compton thick" absorbed AGN (see e.g. the results presented in Della Ceca et al. 1999 from ASCA data); b) objects similar to the class of unabsorbed Seyfert 2 discussed in Pappa et al. (2001); Panessa \& Bassani (2002) and in Barcons et al. (2003). Also the variability could play some role if the nucleus was bright at the time of the XMM-Newton observation but was turned off at the time of the optical spectroscopy. A detailed and exhaustive analysis of these sources is beyond the scope of the present paper. A deeper investigation of their optical (e.g. finer optical classification between Seyfert 1.8, Seyfert 1.9 and Seyfert 2, analysis of the O[III] to 2-10 keV flux ratio) and X-ray (e.g. presence of $\mathrm{Fe} \mathrm{K}_{\alpha}$ emission lines to evaluate the Compton-thickness of the source) properties, as

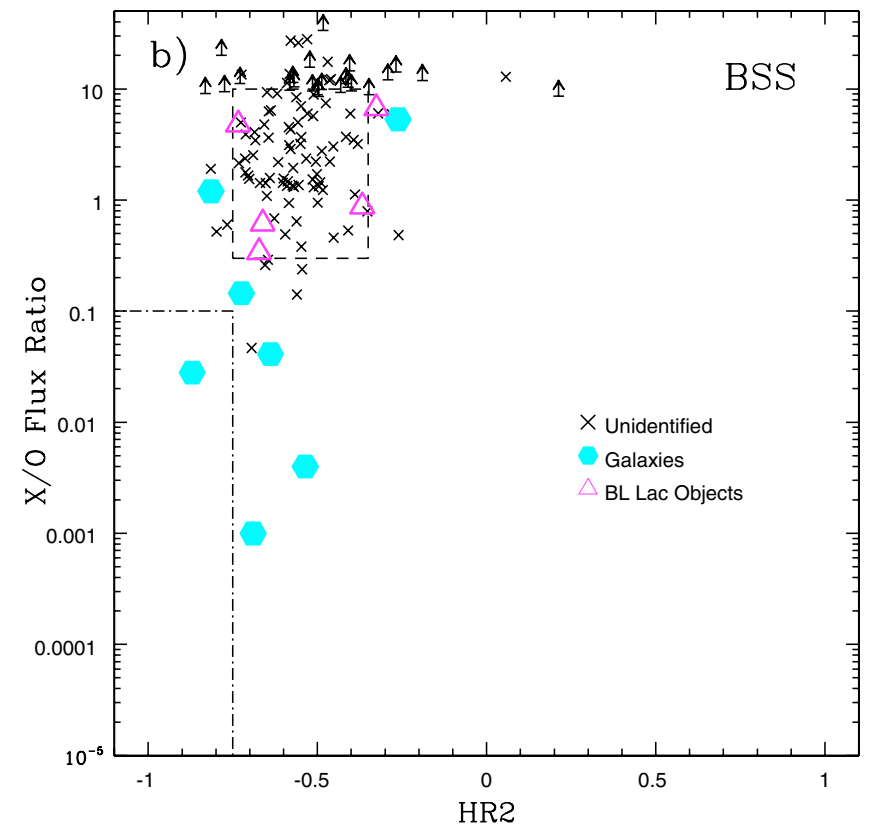

Fig. 6. $H R 2$ vs. X-ray $(0.5-4.5 \mathrm{keV})$ to optical flux ratio for the sources belonging to the BSS sample (panels a) and b)) and to the HBSS sample (panel c)). The X-ray $(0.5-4.5 \mathrm{keV})$ to optical flux ratio for each source has been computed as discussed in Sect. 3.5. We have used different symbols to mark the spectroscopically identified and unidentified objects in the two samples. The box defined by the dot dashed lines (dashed lines) enclose about 95\% (85\%) of the sources optically identified as coronal emitting stars (broad line AGNs) in the BSS sample; these boxes have been reported in all panels to assist with the comparison(s) discussed in the text. The eleven sources belonging only to the HBSS sample are enclosed inside empty squares in panel c).

well as an assessment of the role played by selection effects, is in progress and will be presented elsewhere.

\subsection{X-ray to optical flux ratio}

A useful parameter to discriminate between different classes of $\mathrm{X}$-ray sources is the $\mathrm{X}$-ray to optical flux ratio (X/O flux ratio hereafter; see Maccacaro et al. 1988).

Previous investigations (see Fiore et al. 2003, and references therein) have shown that standard X-ray selected AGN (both type 1 and type 2) have typical X/O flux ratios in the range between 0.1 and 10 (for comparison standard optically selected QSOs and Seyfert 1 galaxies have X/O flux ratio $\sim 1)$. X/O flux ratios below 0.1 are typical of coronal 
emitting stars, normal galaxies (both early type and starforming) and nearby heavily absorbed (Compton thick) AGN. Finally at high X/O flux ratios (well above 10) we can find broad and narrow line AGN as well as high- $z$ high-luminosity obscured AGN (type 2 QSOs), high- $z$ clusters of galaxies and extreme BL Lac objects.

In this paper we have defined the X-ray to optical flux ratio using the observed X-ray flux in the $0.5-4.5 \mathrm{keV}$ energy range and the optical red-band flux (see Fukugita et al. 1995 for the appropriate conversion factors). For the large majority $(73 \%)$ of the objects we have found and used APM red magnitudes. For $19 \%$ of the objects red magnitudes have been found from the literature, measured by us during spectroscopic runs or estimated using magnitudes measured in other optical bands. Finally for the unidentified objects with optical magnitudes below the POSS II limit (30 sources) we have used an upper limit of $\operatorname{mag}_{R} \simeq 21.0$. We note here that, for a fixed X-ray flux, an error of $\sim 1$ magnitude corresponds to an uncertainty of $\sim 60 \%$ on the X/O flux ratio; this uncertainty does not affect any of the general conclusions we discuss below.

In Fig. 6 we have plotted the X/O flux ratio versus the $H R 2$ value for each source (BSS sample in panels a and b; HBSS sample in panel c). The boxes defined by the dot dashed lines (dashed line) indicate the locus of coronal emitting stars (broad line AGN) in the BSS sample and have been reported in all panels to assist with the comparisons.

As is clearly visible in Fig. 6 the bulk of coronal emitting stars is well separated from the bulk of extragalactic sources; some of the AGN (both broad and narrow line) have X/O flux ratio typical of stars but $H R 2$ values typical of AGN; similarly some AGN with an $H R 2$ typical of stars can be distinguished from stars thanks to their X/O flux ratio. Therefore the combined use of $\mathrm{X} / \mathrm{O}$ flux ratio and $H R 2$ allows us to distinguish almost unequivocally galactic sources from the extragalactic ones.

Around $10 \%$ of the extragalactic population have a $\mathrm{X} / \mathrm{O}$ flux ratio greater than 10 . If we consider the $2-10 \mathrm{keV}$ fluxes instead of the $0.5-4.5 \mathrm{keV}$ fluxes this fraction increases to $\sim 15 \%$, in good agreement with the results obtained by Fiore et al. (2003) at fainter fluxes. Amongst the sources identified so far at X/O flux ratio $>10$ there are some broad line and a few narrow line AGN. These results are consistent with those reported in Fiore et al. (2003) who also discuss the observational evidence that at $\mathrm{X} / \mathrm{O}$ flux ratio greater than 10 is where to look for type 2 QSOs. Since the large majority of the X-ray sources at $\mathrm{X} / \mathrm{O}$ flux ratio $>10$ presented here is still unidentified we can not comment further on this; we can only note that many of the unidentified objects with high or very high $\mathrm{X} / \mathrm{O}$ flux ratio seem to have rather standard hardness ratios.

The opposite side of the extragalactic $\mathrm{X} / \mathrm{O}$ distribution $(\mathrm{X} / \mathrm{O}$ flux ratio $<0.1)$ is populated by optically normal galaxies, type 2 AGNs and a few broad line AGN. As discussed above this part of the $\mathrm{X} / \mathrm{O}$ distribution could be also populated by nearby Compton thick AGN; in this respect it is worth noting that a few of the type 2 AGN in the BSS sample populating the same $H R 2$ region of the broad line AGN population have $\mathrm{X} / \mathrm{O}$ flux ratios $<0.1$. In the HBS sample there are also a few

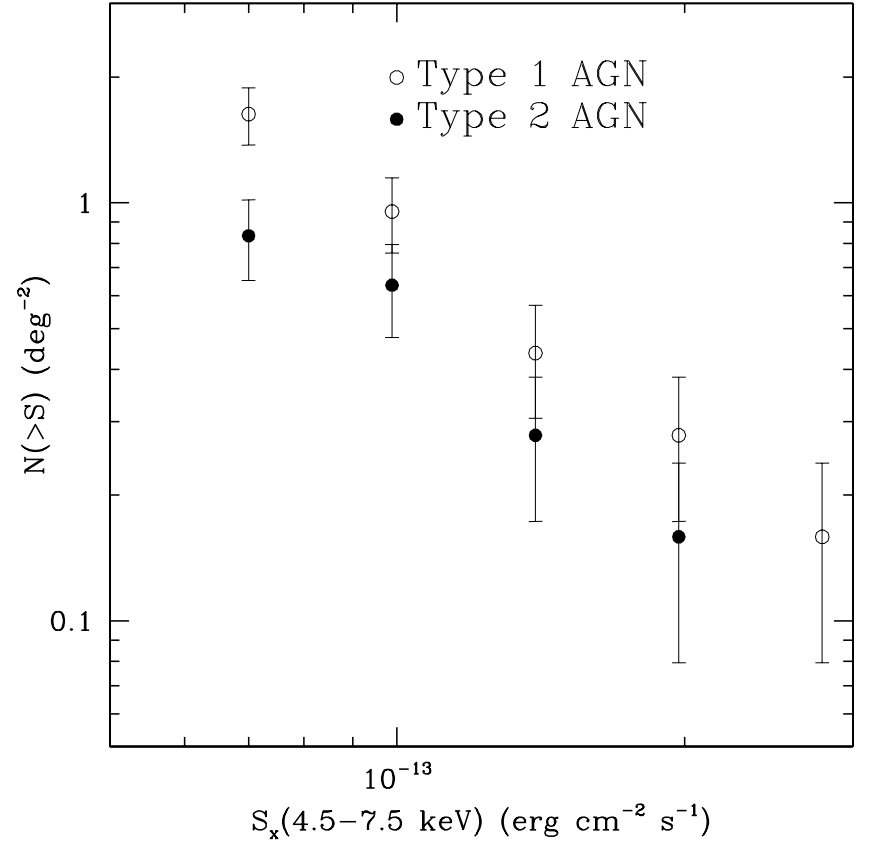

Fig. 7. The number-flux relationship in the $4.5-7.5 \mathrm{keV}$ energy band for optically type 1 (open circles) and type 2 (filled circles) AGN obtained using the sources in the HBSS sample.

type 2 AGN having apparently hard spectra but low X/O flux ratios $(<0.1)$.

\subsection{The number densities of optically broad and narrow line AGN}

The measured X-ray $\log (N>S)-\log S$ of broad and narrow line AGN is a fundamental observable for cosmological investigations and, in particular, provides very strong constraints on the CXB synthesis models and on the history of the accretion in the Universe.

As discussed above the spectroscopic identification rate of the HBSS sample is $\sim 90 \%$, allowing us to investigate for the first time the X-ray $\log (N>S)-\log S$ of optically broad and narrow line AGN in the same sample. In order to deal with the 7 unidentified HBSS sources we have made the reasonable assumption that the 5 unidentified sources having $H R 2>-0.35$ are type 2 AGN, while the 2 unidentified sources having $H R 2$ in the range from -0.75 to -0.35 are type 1 AGN (see Sect. 3.3 for details).

The 4.5-7.5 keV $\log (N>S)-\log S$ of optically broad and narrow line AGN are reported in Fig. 7 (AGN1: open circles; AGN2: filled circles). A conversion factor appropriate for a power-law spectral model with $\alpha_{\mathrm{E}} \simeq 0.7$ has been used to obtain the fluxes. Both $\log N(>S)-\log S$ can be well described by a power-law model and their maximum likelihood best fit parameters have been reported in Table 5 .

At the $4.5-7.5 \mathrm{keV}$ flux limit of $f_{x} \geq \sim 7 \times 10^{-14} \mathrm{erg} \mathrm{cm}^{-2}$ the surface densities of optically type 1 AGN and type 2 AGN are $1.63 \pm 0.25 \mathrm{deg}^{-2}$ and $0.83 \pm 0.18 \mathrm{deg}^{-2}$, respectively. Optically type 2 AGN represent $34 \pm 9 \%$ of the AGN population shining in the $4.5-7.5 \mathrm{keV}$ energy selection band at the 
Table 8. Optically type 2 AGN fraction in few representative X-ray surveys.

\begin{tabular}{lrrrr}
\hline \hline Sample & Energy range & Flux range & Type 2 fraction & Ref./Note \\
$(1)$ & $(2)$ & $(3)$ & $(4)$ & $(5)$ \\
\hline ROSAT Bright Survey & $0.5-2.0$ & $>\sim 2 \times 10^{-12}$ & $1 \pm 0.5 \%$ & $(\mathrm{a})$ \\
XMM BSS & $0.5-4.5$ & $>7 \times 10^{-14}$ & $13 \pm 4 \%$ & $(\mathrm{~b})$ \\
XMM HBSS & $4.5-7.5$ & $>7 \times 10^{-14}$ & $34 \pm 9 \%$ & $(\mathrm{c})$ \\
HELLAS2XMM + Other Surveys & $2-10$ & $10^{-15}-10^{-14}$ & $57 \pm 12 \%$ & $(\mathrm{~d})$ \\
HELLAS2XMM + Other Surveys & $2-10$ & $10^{-14}-10^{-13}$ & $37 \pm 6 \%$ & $(\mathrm{e})$ \\
\hline
\end{tabular}

Columns are as follows: (1) Sample; (2) Energy Selection Band; (3) Investigated flux range; (4) Optically type 2 AGN fraction over the total AGN density in the specific survey; (5) References and Note as follows: (a) From Schwope et al. (2000); (b) From this paper using the BSS sources with Right Ascension below $5^{\mathrm{h}}$ or above $17^{\mathrm{h}}$. For this BSS subsample the spectroscopic identification rate is around $88 \%$; (c) From this paper using the HBSS sample and taking into account the small number of sources still unidentified as discussed in Sect. 3.6; (d) Fraction of optically obscured AGN from Fiore et al. (2003). The spectroscopic ID rate of the sample in this flux range is $\sim 50 \%$; (e) Fraction of optically obscured AGN from Fiore et al. (2003). The spectroscopic ID rate of the sample in this flux range is $\sim 81 \%$.

flux limit of the HBSS. In Table 8 we compare the optically type 2 AGN fraction from the HBSS sample with that found in a few representative X-ray surveys. As expected from the CXB synthesis models the fraction of optically type 2 AGN decreases going to softer X-ray surveys (e.g. by a factor $\sim 2.6$ in the XMM BSS survey and by a factor $\sim 34$ in the ROSAT Bright Survey) while remaining almost constant (around 35\%) for surveys above $2 \mathrm{keV}$ down to a flux limit of $10^{-14} \mathrm{erg} \mathrm{cm}^{-2} \mathrm{~s}^{-1}$. In the flux range between $10^{-15}$ and $10^{-14} \mathrm{erg} \mathrm{cm}^{-2} \mathrm{~s}^{-1}$ there is apparently an increase of the fraction of optically type 2 AGN but the spectroscopic identification rate in this flux range is still low $(\sim 50 \%)$ preventing us from speculating further.

The optically type 2 AGN fraction from the HBSS sample is also in very good agreement with the fraction of X-ray absorbed $\left(N_{\mathrm{H}}>10^{22} \mathrm{~cm}^{-2}\right)$ AGN found from several studies (e.g. Piconcelli et al. 2003; Perola et al. 2004) which ranges between $25 \%$ to $40 \%$ for hard X-ray fluxes spanning four orders of magnitude, from $10^{-10}$ to $10^{-14} \mathrm{erg} \mathrm{cm}^{-2} \mathrm{~s}^{-1}$. While we anticipate that the "Modified Unification Scheme" for the synthesis of the CXB (Ueda et al. 2003) predicts a fraction of absorbed AGN around $41 \%$ for the fluxes (and energy band) covered by the HBSS, a proper comparison will have to wait for the completion of the X-ray spectroscopic work.

\section{Summary and conclusions}

In this paper we have discussed the scientific goals and the strategy of the "The XMM-Newton Bright Serendipitous Survey". This survey comprises two flux-limited samples: the BSS sample and the HBSS sample having a flux limit of $\sim 7 \times 10^{-14} \mathrm{erg} \mathrm{cm}^{-2}$ in the $0.5-4.5 \mathrm{keV}$ and $4.5-7.5 \mathrm{keV}$ energy band, respectively.

From the analysis of 237 suitable XMM-Newton fields (211 for the HBSS), covering a useful survey area of 28.10 (25.17 for the HBSS) sq. deg of sky, we have defined (and presented here) a sample of 400 X-ray sources: 389 of them belong to the BSS, 67 to the HBSS with 56 X-ray sources in common. Up to now $\sim 71 \%(\sim 90 \%)$ of the sources in the BSS (HBSS) sample have been spectroscopically identified, either from the literature or from our spectroscopic observations. The main results from this study can be so summarized:

a) the extragalactic number-flux relationship in the $0.5-4.5 \mathrm{keV}$ and $4.5-7.5 \mathrm{keV}$ band are in good agreement with previous and new results. They are well described by a power law model, $N(>S) \propto S^{-\alpha}$, with best fit value for the slope $\alpha$ of $1.80 \pm 0.11$ and $1.64 \pm 0.24$ in the $0.5-4.5 \mathrm{keV}$ and $4.5-7.5 \mathrm{keV}$ bands, respectively;

b) at the X-ray flux limits of the survey presented here we found that the optical counterpart in the majority $(\sim 90 \%)$ of cases has an optical magnitude brighter than the POSS II limit $\left(R \sim 21^{\mathrm{mag}}\right)$. Galactic counterparts represent about $14 \%$ of the sources in the BSS sample and less than $3 \%$ of the sources in the HBSS sample. The majority of the extragalactic objects identified so far are broad line AGN both in the BSS $(\sim 80 \%)$ and in the HBSS ( 60\%);

c) we have investigated the broad-band spectral properties of the selected sources using hardness ratios. No obvious trend of the source spectra as a function of the count rate is measured. The average spectrum of the "extragalactic" population corresponds to a $(0.5-4.5 \mathrm{keV})$ energy spectral index of $\sim 0.8$ for the BSS sample and $\sim 0.64$ for the HBSS sample. About $13 \%$ (40\%) of the sources in the BSS (HBSS) sample seem to be described by an energy spectral index flatter than that of the $\mathrm{CXB}$; following the results presented in Caccianiga et al. (2004) we speculate that these sources are absorbed AGN with $N_{\mathrm{H}}$ ranging from few times $10^{21}$ up to few times $10^{23} \mathrm{~cm}^{-2}$. There are hints from this study of a significant number of narrow line AGN in the BSS sample occupying the locus typical of X-ray unabsorbed broad line AGN. A deeper investigation of their optical and X-ray properties, of the source selection function, as well as a complete X-ray spectral analysis for all the BSS and HBSS sources using data from all the EPIC instruments (a major goal of this project) is in progress and will be presented elsewhere;

d) we do not find a compelling evidence that the HBSS 4.5-7.5 keV survey is sampling a completely 
different source population compared with the BSS $0.5-4.5 \mathrm{keV}$ survey. Rather we find that the HBSS survey is simply picking up a larger fraction of absorbed AGN, consistent with CXB synthesis models based on the unification scheme of the AGN;

e) at the flux limit of the HBSS sample we measure surface densities of optically classified type 1 and type 2 AGN of $1.63 \pm 0.25 \mathrm{deg}^{-2}$ and $0.83 \pm 0.18 \mathrm{deg}^{-2}$, respectively. The AGN optically classified as type 2 represent $(34 \pm 9) \%$ of the total AGN population shining in the $4.5-7.5 \mathrm{keV}$ energy band at the sampled fluxes. A proper comparison with X-ray absorbed/unabsorbed AGN have to wait for the completion of the ongoing X-ray spectroscopic work.

f) finally, we found a clear separation between Galactic "coronal emitting" stars and extragalactic sources in the hardness ratio diagram and in the (hardness ratio) vs. (X/O flux ratio) diagram. Since the investigated sample is a fair representation of the high Galactic latitude X-ray sky, this result will help with the selection of defined classes of sources from the XMM-Newton catalogue prior to spectroscopic observations making the existing and incoming XMM-Newton catalogs an unique resource for astrophysical studies.

Acknowledgements. This research has made use of the NASA/IPAC Extragalactic Database (NED; which is operated by the Jet Propulsion Laboratory, California Institute of Technology, under contract with the National Aeronautics and Space Administration) and of the SIMBAD database (operated at CDS, Strasbourg, France). We thank Piero Rosati for providing us some IDL routines used in the data analysis and preparation. We thank L. Maraschi, G. Trinchieri, A. Wolter, D. Worrall, N. Webb and the referee for a careful reading of the paper and for useful comments which have improved the paper. We thank Y. Ueda for having provided us with the predictions from the Modified Unification Model in tabular form. R.D.C., T.M., A.C., P.S., V.B. acknowledge partial financial support by the Italian Space Agency (ASI grants: $\mathrm{I} / \mathrm{R} / 037 / 01, \mathrm{I} / \mathrm{R} / 062 / 02$ and $\mathrm{I} / \mathrm{R} / 071 / 02$ ), by the MIUR (Cofin-03-02-23) and INAF. X.B. and F.J.C. acknowledge financial support from the Spanish Ministerio de Ciencia y Tecnología, under the project ESP2003-00812. Based on observations collected at the Italian "Telescopio Nazionale Galileo" (TNG), at the German-Spanish Astronomical Center, Calar Alto (operated jointly by Max-Planck Institut für Astronomie and Instututo de Astrofisica de Andalucia, CSIC), at the European Southern Observatory (ESO) and at the Nordic Optical Telescope (NOT). We would like to thank the staff members of the TNG, ESO, Calar Alto and NOT for their support during the observations. The TNG and NOT telescopes are operated in the island of La Palma by the Nordic Optical Telescope Scientific Association and the Centro Galileo Galilei of the INAF, respectively, in the Spanish Observatorio del Roque de los Muchachos of the Instituto de Astrofísica de Canarias. We finally thank the APM team for maintaining this facility.

\section{References}

Alexander, D. M., Bauer, F. E., Brandt, W. N., et al. 2003, AJ, 126, 539

Baldi, A., Molendi, S., Comastri, A., et al. 2002, ApJ, 564, 190
Barcons, X., Carrera, F. J., \& Ceballos, M. T. 2003, MNRAS, 339, 757

Barcons, X., Carrera, F. J., Watson, M. G., et al. 2002, A\&A, 382, 522 Brandt, W. N., Alexander, D. M., Hornschemeier, A. E., et al. 2001, AJ, 122, 2810

Brandt, W. N., Alexander, D. M., Bauer, F. E., \& Vignali, C. 2004, in Physics of Active Galactic Nuclei at All Scales, ed. D. Alloin, R. Johnson, \& P. Lira (Berlin: Springer-Verlag) [arXiv:astro-ph/0403646]

Caccianiga, A., Severgnini, P., Braito, V., et al. 2004, A\&A, 416, 901

Comastri, A., Setti, G., Zamorani, G., \& Hasinger, G. 1995, A\&A, 296, 1

Comastri, A., Fiore, F., Vignali, C., et al. 2001, MNRAS, 327, 781

Comastri, A., Mignoli, M., Ciliegi, P., et al. 2002, ApJ, 571, 771

Della Ceca, R., Castelli, G., Braito, V., Cagnoni, I., \& Maccacaro, T. 1999, ApJ, 524, 674

Dickey, J. M., \& Lockman, F. J. 1990, ARA\&A, 28, 215

Ehle, M., Breitfellner, M., Gonzales Riestra, R., et al. 2003, XMM-Newton Users' Handbook, Issue 2.1

Elvis, M., Schreier, E. J., Tonry, J., Davis, M., \& Huchra, J. P. 1981, ApJ, 246, 20

Fiore, F., Giommi, P., Vignali, C., et al. 2001, MNRAS, 327, 771

Fiore, F., Brusa, M., Cocchia, F., et al. 2003, A\&A, 409, 79

Fukugita, M., Shimasaku, K., \& Ichikawa, T. 1995, PASP, 107, 945

Galbiati, E., Caccianiga, A., Maccacaro, T., et al. 2004, A\&A, in press

Georgantopoulos, I., Georgakakis, A., Akylas, A., et al. 2004, MNRAS, 352, 91

Gilli, R., Salvati, M., \& Hasinger, G. 2001, A\&A, 366, 407

Gioia, I. M., Maccacaro, T., Schild, R. E., et al. 1990, ApJS, 72, 567

Griffiths, R. E., Georgantopoulos, I., Boyle, B. J., et al. 1995, MNRAS, 275, 77

Hasinger, G., Burg, R., Giacconi, R., et al. 1993, A\&A, 275, 1

Hasinger, G., Burg, R., Giacconi, R., et al. 1998, A\&A, 329, 482

Hasinger, G., Altieri, B., Arnaud, M., et al. 2001, A\&A, 365, L45

Landt, H., Padovani, P., \& Giommi, P. 2002, MNRAS, 336, 945

Maccacaro, T., Gioia, I. M., Zamorani, G., et al. 1982, ApJ, 253, 504

Maccacaro, T., Gioia, I. M., Wolter, A., Zamorani, G., \& Stocke, J. T. 1988, ApJ, 326, 680

Madau, P., Ghisellini, G., \& Fabian, A. C. 1994, MNRAS, 270, 17

Mainieri, V., Bergeron, J., Hasinger, et al. 2002, A\&A, 393, 425

Moretti, A., Campana, S., Lazzati, D., \& Tagliaferri, G. 2003, ApJ, 588,696

Nandra, K., Georgantopoulos, I., Ptak, A., \& Turner, T. J. 2003, ApJ, 582,615

Panessa, F., \& Bassani, L. 2002, A\&A, 394, 435

Pappa, A., Georgantopoulos, I., Stewart, G. C., \& Zezas, A. L. 2001, MNRAS, 326, 995

Perola, G. C., Puccetti, S., Fiore, F., et al. 2004, A\&A, 421, 491

Piconcelli, E., Cappi, M., Bassani, L., Di Cocco, G., \& Dadina, M. 2003, A\&A, 412, 689

Piconcelli, E., Cappi, M., Bassani, L., et al. 2002, A\&A, 394, 835

Rosati, P., Tozzi, P., Giacconi, R., et al. 2002, ApJ, 566, 667

Schwope, A., Hasinger, G., Lehmann, I., et al. 2000, Astron. Nachr., 321,1

Severgnini, P., Caccianiga, A., Braito, et al. 2003, A\&A, 406, 483

Setti, G., \& Woltjer, L. 1989, A\&A, 224, 21

Ueda, Y., Akiyama, M., Ohta, K., \& Miyaji, T. 2003, ApJ, 598, 886

Véron-Cetty, M.-P., \& Véron, P. 2001, A\&A, 374, 92

Yuan, F., \& Narayan, R. 2004, ApJ, 612, 724 
R. Della Ceca et al.: The XMM-Newton bright serendipitous survey, Online Material $p 1$

\section{Online Material}


R. Della Ceca et al.: The XMM-Newton bright serendipitous survey, Online Material p 2

Table 2. Basic information on the XMM-Newton MOS2 fields used for the sample selection.

\begin{tabular}{|c|c|c|c|c|c|c|c|c|}
\hline Obs. ID & Filter & RA; Dec (J2000) & $\begin{array}{r}\text { Exposure } \\
\mathrm{s} \\
(4)\end{array}$ & $\begin{array}{l}\log N h \\
\mathrm{~cm}^{-2} \\
(5)\end{array}$ & $\begin{array}{l}R_{\text {in }} \\
\operatorname{arcmin} \\
(6)\end{array}$ & $\begin{array}{l}R_{\text {out }} \\
\text { arcmin } \\
(7)\end{array}$ & $\begin{array}{l}\text { BSS } \\
\text { sres } \\
(8)\end{array}$ & $\begin{array}{l}\text { HBSS } \\
\text { srcs } \\
(9)\end{array}$ \\
\hline 0125310101 & Medium & $000030.4-250643.4$ & 19162.6 & 20.27 & 1 & 13 & 4 & 0 \\
\hline 0101040101 & Medium & $000619.7+201222.8$ & 34044.7 & 20.60 & 8 & 13 & 1 & 0 \\
\hline 0127110201 & Thin1 & $001031.2+105840.7$ & 7558.0 & 20.76 & 2 & 13 & 2 & 0 \\
\hline 0111000101 & Medium & $001833.0+162608.0$ & 31362.1 & 20.61 & 3 & 13 & 2 & 0 \\
\hline 0001930101 & Thin1 & $002606.8+104112.5$ & 18032.3 & 20.71 & 1 & 13 & 1 & 1 \\
\hline 0050140201 & Thin1 & $002635.9+170937.8$ & 50380.4 & 20.63 & 3 & 13 & 2 & 0 \\
\hline 0112320101 & Thin1 & $003027.6+045147.4$ & 24577.8 & 20.48 & 1 & 13 & 1 & 0 \\
\hline 0065770101 & Medium & $003247.0+393433.3$ & 7360.0 & 20.82 & 1 & 13 & 1 & 0 \\
\hline 0125920201 & Thin1 & $003354.5-120710.8$ & 9709.9 & 20.40 & 1 & 13 & 3 & 0 \\
\hline $0125320701^{*}$ & Thin1 & $005003.0-520736.7$ & 16524.9 & 20.51 & 1 & 13 & 3 & - \\
\hline 0110890401 & Medium & $005720.0-222304.1$ & 29741.8 & 20.19 & 8 & 13 & 0 & 0 \\
\hline 0111280301 & Thin1 & $005851.3-273547.1$ & 7975.6 & 20.28 & 0 & 12 & 1 & 0 \\
\hline 0112650401 & Medium & $010424.0-062410.6$ & 23695.9 & 20.71 & 0 & 13 & 1 & 0 \\
\hline 0103861601 & Thick & $010516.4-582610.4$ & 7127.1 & 20.44 & 8 & 13 & 1 & 0 \\
\hline 0025540101 & Medium & $010746.5-173014.0$ & 7678.2 & 20.18 & 2 & 13 & 2 & 0 \\
\hline $0113040801^{*}$ & Thin1 & $012039.7-105624.5$ & 5692.0 & 20.51 & 1 & 13 & 4 & - \\
\hline 0101040201 & Medium & $012346.0-584825.8$ & 28994.1 & 20.50 & 8 & 13 & 0 & 0 \\
\hline 0109860101 & Thin1 & $012533.4+014538.2$ & 38533.6 & 20.49 & 4 & 13 & 2 & 0 \\
\hline $0112600601^{*}$ & Medium & $012732.1+191032.3$ & 3994.2 & 20.68 & 8 & 13 & 3 & - \\
\hline 0084230301 & Medium & $013153.7-133656.4$ & 20446.4 & 20.21 & 8 & 13 & 1 & 1 \\
\hline 0112630201 & Thin1 & $013301.9-400623.5$ & 31094.2 & 20.28 & 1 & 13 & 1 & 0 \\
\hline 0111430101 & Medium & $013901.2-175706.3$ & 33440.3 & 20.16 & 8 & 13 & 1 & 0 \\
\hline 0032140401 & Medium & $014012.1-674840.3$ & 7610.7 & 20.40 & 0 & 13 & 4 & 2 \\
\hline 0093641001 & Medium & $014301.7+133824.4$ & 9329.1 & 20.69 & 4 & 13 & 2 & 0 \\
\hline 0101640201 & Medium & $015950.2+002346.8$ & 7543.3 & 20.41 & 8 & 13 & 3 & 1 \\
\hline 0084140101 & Medium & $020838.2+352300.2$ & 38001.6 & 20.80 & 2 & 13 & 3 & 0 \\
\hline 0112371701 & Thin1 & $021712.4-043904.2$ & 20001.1 & 20.38 & 0 & 11 & 2 & 1 \\
\hline 0112371001 & Thin 1 & $021800.3-045947.8$ & 50800.3 & 20.40 & 0 & 11 & 4 & 3 \\
\hline $0112371501^{*}$ & Thin 1 & $021848.3-043902.9$ & 6684.2 & 20.40 & 0 & 11 & 0 & - \\
\hline 0112370301 & Thin1 & $021936.3-045947.7$ & 50384.1 & 20.41 & 0 & 11 & 1 & 0 \\
\hline 0111110501 & Thin1 & $022240.0-050949.0$ & 24185.8 & 20.41 & 0 & 10 & 0 & 0 \\
\hline 0109520501 & Thin 1 & $022320.0-044949.3$ & 24107.8 & 20.42 & 0 & 10 & 1 & 0 \\
\hline 0111110401 & Thin1 & $022400.1-050953.6$ & 28276.2 & 20.42 & 0 & 10 & 1 & 0 \\
\hline 0111110301 & Thin1 & $022520.3-050952.5$ & 24158.7 & 20.43 & 0 & 10 & 0 & 0 \\
\hline 0111110201 & Thin1 & $022640.1-050947.1$ & 8229.8 & 20.42 & 0 & 10 & 1 & 0 \\
\hline 0111110101 & Thin1 & $022760.0-050948.4$ & 23809.9 & 20.42 & 0 & 10 & 0 & 0 \\
\hline 0103861101 & Medium & $023512.8-293609.9$ & 7409.2 & 20.25 & 8 & 13 & 1 & 0 \\
\hline 0098810101 & Thin1 & $023612.3-521955.6$ & 23450.3 & 20.52 & 2 & 13 & 2 & 1 \\
\hline 0075940301 & Medium & $023657.9+243853.7$ & 47426.5 & 20.96 & 8 & 13 & 0 & 0 \\
\hline 0067190101 & Medium & $023819.4-521134.1$ & 25627.4 & 20.50 & 3 & 10 & 1 & 0 \\
\hline 0111200101 & Medium & $024241.1-000053.7$ & 35485.0 & 20.55 & 8 & 13 & 4 & 0 \\
\hline 0111490401 & Medium & $024843.8+310659.2$ & 31028.6 & 20.97 & 8 & 13 & 0 & 0 \\
\hline 0056020301 & Thin1 & $025632.8+000601.7$ & 17250.5 & 20.81 & 2 & 13 & 2 & 0 \\
\hline 0041170101 & Thin1 & $030238.5+000731.9$ & 47275.7 & 20.85 & 0 & 13 & 2 & 1 \\
\hline 0042340501 & Thin1 & $030703.8-284024.1$ & 13285.0 & 20.13 & 4 & 13 & 2 & 1 \\
\hline 0122520201 & Thick & $031159.3-765153.0$ & 28295.0 & 20.91 & 2 & 13 & 2 & 1 \\
\hline 0110970101 & Thin 1 & $031309.6-550348.4$ & 10239.4 & 20.41 & 0 & 13 & 2 & 1 \\
\hline 0110970401 & Thin 1 & $031415.9-552133.5$ & 8457.5 & 20.42 & 0 & 8 & 0 & 0 \\
\hline 0129320901 & Thin1 & $031603.7-550608.5$ & 9948.2 & 20.43 & 0 & 13 & 1 & 0 \\
\hline 0105660101 & Medium & $031756.0-441415.1$ & 23150.7 & 20.41 & 6 & 13 & 2 & 1 \\
\hline 0108060501 & Thin1 & $033229.1-274827.1$ & 46833.4 & 19.95 & 0 & 13 & 2 & 0 \\
\hline 0099010101 & Thin1 & $033527.6-254454.5$ & 18776.9 & 20.01 & 8 & 13 & 3 & 0 \\
\hline 0055140101 & Thin1 & $033935.0-352558.1$ & 47634.6 & 20.12 & 1 & 11 & 3 & 1 \\
\hline 0111970301 & Thin1 & $040907.2-711743.9$ & 18740.3 & 20.88 & 8 & 13 & 3 & 3 \\
\hline 0112600401 & Medium & $042544.2-571334.4$ & 7627.0 & 20.31 & 8 & 13 & 1 & 0 \\
\hline 0103861701 & Medium & $043517.1-780154.3$ & 8010.5 & 20.88 & 8 & 13 & 1 & 0 \\
\hline 0112880401 & Thick & $045935.4+014716.0$ & 18914.3 & 20.89 & 2 & 13 & 2 & 0 \\
\hline
\end{tabular}


Table 2. continued.

\begin{tabular}{|c|c|c|c|c|c|c|c|c|}
\hline Obs. ID & Filter & RA; Dec (J2000) & $\begin{array}{r}\text { Exposure } \\
\mathrm{s} \\
(4)\end{array}$ & $\begin{array}{l}\log N h \\
\mathrm{~cm}^{-2} \\
(5)\end{array}$ & $\begin{array}{l}R_{\text {in }} \\
\operatorname{arcmin} \\
(6) \\
\end{array}$ & $\begin{array}{l}R_{\text {out }} \\
\operatorname{arcmin} \\
(7) \\
\end{array}$ & $\begin{array}{l}\text { BSS } \\
\text { srcs } \\
(8) \\
\end{array}$ & $\begin{array}{l}\text { HBSS } \\
\text { srcs } \\
(9) \\
\end{array}$ \\
\hline 0111160201 & Thin1 & $050520.0-284857.6$ & 47984.4 & 20.17 & 2 & 13 & 4 & 1 \\
\hline $0103860701^{*}$ & Medium & $051621.3-103334.5$ & 4740.3 & 20.97 & 8 & 13 & 1 & - \\
\hline 0094400101 & Medium & $051640.1+794016.7$ & 31727.2 & 20.90 & 2 & 13 & 4 & 0 \\
\hline 0090050701 & Thin1 & $051949.4-454648.9$ & 13426.7 & 20.62 & 8 & 13 & 2 & 0 \\
\hline 0085640101 & Thin1 & $052100.9-252152.6$ & 9720.6 & 20.28 & 1 & 13 & 5 & 2 \\
\hline 0050150101 & Thin1 & $052505.9-334313.3$ & 13440.2 & 20.35 & 1 & 13 & 2 & 0 \\
\hline $0123720301^{*}$ & Medium & $052844.1-652658.6$ & 4762.6 & 20.73 & 8 & 13 & 0 & - \\
\hline 0009220601 & Medium & $061535.2+710212.2$ & 11376.9 & 20.92 & 2 & 13 & 1 & 0 \\
\hline 0110930101 & Medium & $061841.9+782125.7$ & 19578.2 & 20.86 & 5 & 13 & 0 & 0 \\
\hline 0103860101 & Medium & $062308.8-643615.8$ & 8862.8 & 20.73 & 8 & 13 & 2 & 0 \\
\hline 0029340101 & Medium & $064118.4+821559.3$ & 22288.1 & 20.70 & 8 & 13 & 1 & 0 \\
\hline 0061540101 & Medium & $065211.4+742530.1$ & 21080.3 & 20.81 & 2 & 13 & 3 & 0 \\
\hline 0112980201 & Thin 1 & $065817.6-555730.6$ & 41042.1 & 20.81 & 8 & 13 & 2 & 0 \\
\hline 0083000101 & Medium & $073519.9+434427.3$ & 13589.8 & 20.77 & 2 & 13 & 0 & 0 \\
\hline $0103862101^{*}$ & Medium & $074232.8+494830.4$ & 5909.3 & 20.75 & 8 & 13 & 1 & - \\
\hline 0123100101 & Thin1 & $074403.3+743342.9$ & 39171.0 & 20.55 & 3 & 13 & 4 & 2 \\
\hline 0111100301 & Thin 1 & $075109.3+180743.0$ & 32111.2 & 20.61 & 1 & 13 & 1 & 0 \\
\hline 0110070401 & Thin1 & $075505.0+220004.6$ & 22449.2 & 20.75 & 8 & 13 & 0 & 0 \\
\hline 0094400301 & Medium & $080434.3+645955.6$ & 19077.9 & 20.64 & 2 & 13 & 2 & 1 \\
\hline 0094530401 & Thin1 & $080535.2+244956.7$ & 8709.6 & 20.59 & 0 & 13 & 2 & 0 \\
\hline 0092800101 & Medium & $083142.4+524520.8$ & 16808.2 & 20.59 & 2 & 13 & 1 & 0 \\
\hline 0025540301 & Medium & $083822.8+254513.9$ & 11803.5 & 20.56 & 1 & 13 & 4 & 2 \\
\hline 0111400101 & Thick & $083911.6+650112.1$ & 24224.9 & 20.63 & 8 & 13 & 1 & 0 \\
\hline $0112620101^{*}$ & Medium & $084122.9+705337.8$ & 4301.9 & 20.47 & 8 & 13 & 0 & - \\
\hline 0103660201 & Medium & $084742.4+344507.3$ & 16223.3 & 20.52 & 8 & 13 & 1 & 0 \\
\hline $0085030101^{*}$ & Thin1 & $085535.7+584405.8$ & 6545.6 & 20.62 & 5 & 13 & 2 & - \\
\hline 0110660201 & Medium & $090602.5+620302.2$ & 8671.8 & 20.65 & 0 & 13 & 1 & 0 \\
\hline 0083240201 & Thin1 & $091128.2+055057.3$ & 19349.7 & 20.56 & 2 & 13 & 1 & 0 \\
\hline 0084230601 & Medium & $091752.1+514333.1$ & 16347.4 & 20.16 & 6 & 13 & 1 & 1 \\
\hline 0021740101 & Medium & $093953.7+355406.4$ & 10323.5 & 20.16 & 2 & 13 & 0 & 0 \\
\hline 0106460101 & Thin 1 & $094301.2+465934.4$ & 49423.9 & 20.10 & 3 & 13 & 0 & 0 \\
\hline 0017540101 & Medium & $094525.9-083903.2$ & 7573.9 & 20.56 & 8 & 13 & 2 & 0 \\
\hline 0111290101 & Thin1 & $095048.7+392659.8$ & 21359.3 & 20.20 & 8 & 13 & 1 & 0 \\
\hline 0065790101 & Thin1 & $095209.1-014814.6$ & 9322.6 & 20.60 & 2 & 13 & 2 & 1 \\
\hline $0070940101^{*}$ & Thin 1 & $095341.5+013455.9$ & 6021.1 & 20.55 & 5 & 13 & 2 & - \\
\hline 0112850101 & Thin1 & $095456.3+174329.7$ & 17345.1 & 20.50 & 1 & 13 & 2 & 0 \\
\hline 0111290201 & Thin1 & $095652.6+411524.9$ & 12896.7 & 19.90 & 8 & 13 & 1 & 0 \\
\hline 0041170201 & Medium & $100040.8+251424.8$ & 46751.9 & 20.44 & 0 & 13 & 3 & 0 \\
\hline 0110930201 & Medium & $100156.9+554041.1$ & 7317.1 & 19.90 & 2 & 13 & 2 & 0 \\
\hline 0070340201 & Thin 1 & $100847.1+534200.8$ & 21851.2 & 19.87 & 1 & 13 & 3 & 0 \\
\hline 0085170101 & Thin1 & $101113.5+554455.0$ & 31755.9 & 19.89 & 2 & 13 & 0 & 0 \\
\hline $0086750101^{*}$ & Thin1 & $101615.4+520924.1$ & 6574.0 & 19.88 & 2 & 13 & 3 & - \\
\hline 0028740301 & Thin1 & $101840.5+412515.2$ & 28448.5 & 20.05 & 8 & 13 & 4 & 2 \\
\hline 0093640301 & Medium & $101959.8+081332.3$ & 12804.9 & 20.48 & 1 & 13 & 2 & 0 \\
\hline 0101040301 & Medium & $102330.7+195153.1$ & 36611.7 & 20.33 & 4 & 13 & 1 & 0 \\
\hline 0108670101 & Thin1 & $102339.9+041125.6$ & 50918.8 & 20.48 & 8 & 13 & 2 & 0 \\
\hline 0102040301 & Thick & $103059.0+310251.8$ & 26293.5 & 20.29 & 8 & 13 & 2 & 0 \\
\hline 0110950101 & Thin1 & $103154.2-141652.5$ & 7308.6 & 20.82 & 8 & 13 & 0 & 0 \\
\hline $0112810301^{*}$ & Thin1 & $103844.4+533008.4$ & 4917.1 & 20.05 & 3 & 13 & 2 & - \\
\hline 0059800101 & Thin1 & $103953.7+205052.2$ & 14293.2 & 20.31 & 2 & 13 & 5 & 1 \\
\hline 0125300101 & Thin1 & $104432.4-012505.3$ & 32227.5 & 20.62 & 2 & 13 & 4 & 1 \\
\hline 0055990201 & Thin1 & $104953.6+325858.1$ & 19840.1 & 20.30 & 4 & 13 & 2 & 1 \\
\hline
\end{tabular}


Table 2. continued.

\begin{tabular}{|c|c|c|c|c|c|c|c|c|}
\hline$\overline{\text { Obs. ID }}$ & Filter & RA; Dec (J2000) & $\begin{array}{r}\text { Exposure } \\
\mathrm{S} \\
\text { (4) }\end{array}$ & $\begin{array}{l}\log N h \\
\mathrm{~cm}^{-2} \\
(5)\end{array}$ & $\begin{array}{l}R_{\text {in }} \\
\operatorname{arcmin} \\
(6)\end{array}$ & $\begin{array}{l}R_{\text {out }} \\
\text { arcmin } \\
(7)\end{array}$ & $\begin{array}{l}\text { BSS } \\
\text { srcs } \\
(8)\end{array}$ & $\begin{array}{l}\text { HBSS } \\
\text { srcs } \\
(9)\end{array}$ \\
\hline 0022740101 & Medium & $105244.1+572848.7$ & 82880.4 & 19.75 & 0 & 13 & 5 & 0 \\
\hline 0094800101 & Thin1 & $105659.5-033729.8$ & 31924.0 & 20.55 & 2 & 13 & 1 & 0 \\
\hline $0110660301^{*}$ & Thin1 & $110108.4+860959.2$ & 6216.2 & 20.83 & 1 & 13 & 0 & - \\
\hline 0112880201 & Medium & $110152.3-344227.4$ & 28570.1 & 20.70 & 2 & 13 & 3 & 1 \\
\hline $0070340301^{*}$ & Medium & $110336.6+355511.8$ & 8386.3 & 20.30 & 1 & 13 & 1 & - \\
\hline 0112630101 & Thin1 & $110633.6-182135.1$ & 12627.6 & 20.66 & 1 & 13 & 1 & 0 \\
\hline 0099030101 & Thin1 & $111718.6+175750.3$ & 20091.0 & 20.18 & 1 & 13 & 1 & 0 \\
\hline 0111290401 & Thin 1 & $111908.8+211917.4$ & 8525.8 & 20.11 & 8 & 13 & 2 & 0 \\
\hline 0107860201 & Thin1 & $112006.3+431759.9$ & 22162.9 & 20.32 & 1 & 13 & 0 & 1 \\
\hline 0093641101 & Medium & $112015.0+125929.1$ & 10566.2 & 20.39 & 5 & 13 & 3 & 0 \\
\hline 0112270301 & Thin1 & $112221.7+241739.2$ & 14296.9 & 20.10 & 6 & 13 & 0 & 0 \\
\hline 0110660401 & Medium & $112600.6+425305.1$ & 13085.5 & 20.32 & 1 & 13 & 0 & 0 \\
\hline 0112810101 & Thin1 & $112829.1+583343.7$ & 20593.3 & 20.00 & 3 & 13 & 0 & 0 \\
\hline 0102040201 & Thick & $113109.2+311408.0$ & 23552.4 & 20.31 & 8 & 13 & 3 & 3 \\
\hline 0042341001 & Thin1 & $113156.2-195532.8$ & 12683.8 & 20.65 & 6 & 13 & 1 & 0 \\
\hline 0112210101 & Medium & $113902.3-374417.2$ & 37360.0 & 20.93 & 4 & 13 & 1 & 0 \\
\hline 0094800201 & Thin1 & $114023.9+660837.6$ & 24709.2 & 20.09 & 1 & 13 & 0 & 0 \\
\hline $0112551401^{*}$ & Thin1 & $115254.0+365906.6$ & 6471.1 & 20.28 & 4 & 13 & 1 & - \\
\hline 0090020101 & Thin1 & $115755.3+552707.8$ & 10362.7 & 20.09 & 3 & 13 & 1 & 0 \\
\hline 0109141401 & Medium & $120309.4+443152.4$ & 62156.3 & 20.12 & 8 & 13 & 2 & 0 \\
\hline 0112310101 & Medium & $121032.3+392419.8$ & 30192.1 & 20.30 & 8 & 13 & 0 & 0 \\
\hline 0112610101 & Medium & $121417.0+140316.9$ & 51088.9 & 20.44 & 8 & 13 & 1 & 0 \\
\hline $0111840101^{*}$ & Medium & $122122.2+301038.1$ & 9637.0 & 20.24 & 4 & 13 & 0 & - \\
\hline 0124110101 & Medium & $122141.5+751838.0$ & 17009.1 & 20.46 & 2 & 13 & 2 & 0 \\
\hline 0108860101 & Thin1 & $122307.2+103715.1$ & 20960.4 & 20.35 & 4 & 13 & 0 & 0 \\
\hline 0070340501 & Thin1 & $122715.6+333218.9$ & 15390.5 & 20.14 & 1 & 13 & 4 & 0 \\
\hline 0110990201 & Thin1 & $122718.4+012920.2$ & 11954.5 & 20.26 & 1 & 13 & 2 & 1 \\
\hline 0126700301 & Medium & $122906.6+020308.0$ & 56647.6 & 20.25 & 8 & 13 & 2 & 0 \\
\hline 0112650101 & Medium & $123111.4+204752.2$ & 23562.4 & 20.30 & 0 & 13 & 0 & 0 \\
\hline 0124900101 & Thin1 & $123130.9+641420.0$ & 33644.1 & 20.30 & 2 & 13 & 4 & 0 \\
\hline 0006220201 & Thick & $123534.9-395455.9$ & 42741.5 & 20.86 & 1 & 13 & 2 & 1 \\
\hline 0111550101 & Thin1 & $123648.5+621256.4$ & 43468.9 & 20.17 & 0 & 13 & 3 & 0 \\
\hline 0109970101 & Medium & $123939.1-052040.3$ & 8087.8 & 20.36 & 4 & 13 & 0 & 0 \\
\hline 0136950201 & Thin1 & $124237.9-111922.4$ & 29166.8 & 20.56 & 1 & 13 & 1 & 0 \\
\hline 0111190701 & Medium & $124248.1+024117.8$ & 59825.8 & 20.26 & 8 & 13 & 0 & 0 \\
\hline 0051760101 & Thin1 & $124634.9+022201.1$ & 12244.0 & 20.24 & 2 & 13 & 4 & 1 \\
\hline 0060370201 & Thin1 & $124913.5-055927.5$ & 40156.8 & 20.33 & 1 & 13 & 4 & 0 \\
\hline 0008220201 & Thick & $125141.5+273225.3$ & 39747.7 & 19.95 & 8 & 13 & 0 & 0 \\
\hline 0111020101 & Medium & $125302.9-292341.4$ & 29008.5 & 20.78 & 8 & 13 & 0 & 0 \\
\hline 0081340201 & Medium & $125613.6+565224.0$ & 21493.1 & 20.10 & 1 & 13 & 2 & 0 \\
\hline 0017940101 & Thin1 & $130543.5+180059.0$ & 50660.2 & 20.32 & 8 & 13 & 1 & 0 \\
\hline 0002940101 & Medium & $130706.6-234027.2$ & 7263.6 & 20.96 & 1 & 13 & 2 & 0 \\
\hline 0093640401 & Medium & $132035.4+340821.4$ & 19663.3 & 20 & 1 & 13 & 4 & 1 \\
\hline 0100240201 & Medium & $133047.4+241359.1$ & 35954.0 & 20.06 & 3 & 13 & 3 & 0 \\
\hline 0061940101 & Medium & $133152.9+111643.9$ & 7011.7 & 20.29 & 1 & 13 & 1 & 0 \\
\hline 0111160101 & Thin1 & $133419.2+503055.6$ & 47144.5 & 20.04 & 2 & 13 & 1 & 0 \\
\hline 0111570201 & Medium & $133553.9-341744.3$ & 50435.3 & 20.61 & 6 & 13 & 1 & 0 \\
\hline 0096010101 & Thin1 & $133718.8+242302.3$ & 32367.8 & 20.06 & 8 & 13 & 1 & 0 \\
\hline 0035940301 & Medium & $133955.8-313830.3$ & 42378.9 & 20.59 & 5 & 13 & 1 & 1 \\
\hline 0112250201 & Thin1 & $134740.0+581245.2$ & 33008.0 & 20.11 & 1 & 13 & 2 & 2 \\
\hline 0098010101 & Thin1 & $140102.0+025244.3$ & 32776.7 & 20.37 & 5 & 13 & 1 & 2 \\
\hline 0109910101 & Thin1 & $140134.5-110740.3$ & 48892.6 & 20.63 & 6 & 13 & 3 & 1 \\
\hline 0092850501 & Thin1 & $140923.9+261821.4$ & 41167.0 & 20.17 & 2 & 13 & 2 & 0 \\
\hline 0013140101 & Medium & $141314.9-031225.5$ & 17838.3 & 20.58 & 8 & 13 & 1 & 0 \\
\hline
\end{tabular}


Table 2. continued.

\begin{tabular}{|c|c|c|c|c|c|c|c|c|}
\hline Obs. ID & Filter & $\overline{\text { RA; Dec (J2000) }}$ & $\begin{array}{r}\text { Exposure } \\
\mathrm{s} \\
(4)\end{array}$ & $\begin{array}{l}\log N h \\
\mathrm{~cm}^{-2} \\
(5)\end{array}$ & $\begin{array}{l}R_{\text {in }} \\
\operatorname{arcmin} \\
(6) \\
\end{array}$ & $\begin{array}{l}R_{\text {out }} \\
\operatorname{arcmin} \\
(7) \\
\end{array}$ & $\begin{array}{l}\text { BSS } \\
\text { srcs } \\
(8) \\
\end{array}$ & $\begin{array}{l}\text { HBSS } \\
\text { srcs } \\
(9) \\
\end{array}$ \\
\hline 0112250301 & Thin1 & $141545.6+112935.7$ & 25593.4 & 20.26 & 2 & 13 & 2 & 1 \\
\hline 0127921001 & Thin1 & $141710.3+522354.1$ & 54477.7 & 20.11 & 0 & 13 & 3 & 0 \\
\hline 0109960101 & Medium & $141759.2+250811.7$ & 23142.3 & 20.23 & 4 & 13 & 3 & 1 \\
\hline 0111850201 & Medium & $142832.5+424016.4$ & 39671.3 & 20.14 & 4 & 13 & 2 & 1 \\
\hline 0111530101 & Thick & $143859.9+641725.5$ & 50080.5 & 20.23 & 8 & 13 & 3 & 2 \\
\hline 0057560301 & Thin1 & $144929.2+085951.1$ & 38812.8 & 20.31 & 0 & 13 & 2 & 0 \\
\hline 0056030101 & Medium & $145122.8+190602.7$ & 58940.0 & 20.39 & 8 & 13 & 0 & 0 \\
\hline 0067750101 & Thin1 & $145821.3-314009.0$ & 34424.0 & 20.93 & 2 & 13 & 1 & 0 \\
\hline 0070740301 & Thin1 & $150401.1+102615.0$ & 10625.1 & 20.37 & 8 & 13 & 1 & 0 \\
\hline 0018741001 & Thin1 & $151846.4+061410.4$ & 7406.8 & 20.51 & 4 & 13 & 2 & 0 \\
\hline 0100240801 & Medium & $153223.0-083201.3$ & 23738.7 & 20.96 & 3 & 13 & 2 & 0 \\
\hline 0091140401 & Thin1 & $153228.6+044048.6$ & 34556.1 & 20.62 & 5 & 13 & 0 & 0 \\
\hline 0112190401 & Medium & $153501.4+012045.4$ & 13512.1 & 20.69 & 2 & 13 & 3 & 1 \\
\hline $0094380801^{*}$ & Medium & $155543.2+111116.3$ & 5805.5 & 20.56 & 8 & 13 & 0 & - \\
\hline 0067340601 & Medium & $160713.8+080451.5$ & 14215.2 & 20.60 & 0 & 13 & 3 & 1 \\
\hline 0103460801 & Thin 1 & $161628.7+121222.1$ & 13130.2 & 20.66 & 1 & 13 & 2 & 0 \\
\hline 0103461001 & Thin 1 & $161736.8+123657.7$ & 11734.5 & 20.66 & 1 & 13 & 1 & 1 \\
\hline $0061940301^{*}$ & Medium & $163053.6+781054.5$ & 4965.0 & 20.61 & 2 & 13 & 6 & - \\
\hline 0112230301 & Medium & $163245.7+053439.6$ & 20662.8 & 20.75 & 8 & 13 & 1 & 0 \\
\hline $0049540101^{*}$ & Medium & $163420.8+570943.1$ & 10855.0 & 20.27 & 2 & 13 & 2 & - \\
\hline 0067340501 & Medium & $164217.3+031057.5$ & 14421.1 & 20.74 & 0 & 13 & 1 & 0 \\
\hline 0101640101 & Medium & $165258.4+022400.4$ & 16112.3 & 20.76 & 8 & 13 & 1 & 0 \\
\hline 0113070101 & Medium & $165359.8+141752.6$ & 7298.1 & 20.74 & 0 & 13 & 4 & 2 \\
\hline 0111060101 & Medium & $165749.6+352035.8$ & 9421.0 & 20.25 & 3 & 13 & 2 & 0 \\
\hline $0102040101^{*}$ & Thick & $172320.8+341800.6$ & 6893.5 & 20.49 & 8 & 13 & 1 & - \\
\hline $0067340101^{*}$ & Medium & $185537.1-463057.6$ & 10652.7 & 20.72 & 0 & 11 & 2 & - \\
\hline 0081341001 & Thin1 & $193121.7-723913.3$ & 15287.3 & 20.77 & 1 & 13 & 2 & 1 \\
\hline 0081340501 & Medium & $201330.0-414725.7$ & 19195.9 & 20.69 & 0 & 13 & 0 & 0 \\
\hline 0111180201 & Medium & $204010.0-005214.7$ & 16494.9 & 20.83 & 8 & 13 & 2 & 1 \\
\hline 0111510101 & Medium & $204150.9-322619.8$ & 14918.6 & 20.71 & 8 & 13 & 3 & 0 \\
\hline $0130720201^{*}$ & Thin1 & $204409.0-104311.2$ & 5311.7 & 20.61 & 8 & 13 & 0 & - \\
\hline 0111420101 & Medium & $204509.4-312036.6$ & 42956.6 & 20.74 & 8 & 13 & 0 & 0 \\
\hline 0112600501 & Medium & $204620.0-024848.4$ & 10549.7 & 20.70 & 8 & 13 & 1 & 0 \\
\hline 0083210101 & Thin1 & $205418.8-155538.2$ & 10434.9 & 20.64 & 2 & 13 & 2 & 0 \\
\hline 0112190601 & Medium & $205621.6-043759.4$ & 16639.7 & 20.70 & 2 & 13 & 1 & 1 \\
\hline 0081340401 & Thin1 & $205827.1-423856.9$ & 15001.6 & 20.59 & 1 & 13 & 2 & 1 \\
\hline 0041150101 & Medium & $210411.1-112140.0$ & 38734.2 & 20.67 & 1 & 13 & 1 & 0 \\
\hline 0038540301 & Thin1 & $210440.2-122005.6$ & 14697.9 & 20.65 & 2 & 13 & 1 & 0 \\
\hline 0088020201 & Thin1 & $212738.1-444838.5$ & 16093.9 & 20.54 & 1 & 13 & 2 & 0 \\
\hline 0103060101 & Medium & $212912.2-153834.7$ & 22041.8 & 20.70 & 3 & 13 & 1 & 1 \\
\hline 0092850201 & Medium & $213745.3-143256.4$ & 16014.8 & 20.67 & 2 & 13 & 1 & 1 \\
\hline 0109463501 & Thin1 & $213756.5-434219.8$ & 7577.3 & 20.46 & 2 & 13 & 3 & 0 \\
\hline $0061940201^{*}$ & Medium & $213807.9-423606.6$ & 4867.9 & 20.43 & 1 & 13 & 5 & - \\
\hline 0008830101 & Thin1 & $214015.5-233932.1$ & 13986.0 & 20.55 & 2 & 13 & 1 & 1 \\
\hline 0103060401 & Medium & $215155.9-302743.5$ & 24071.7 & 20.33 & 3 & 13 & 2 & 0 \\
\hline 0111270101 & Thin1 & $215337.3+174157.7$ & 13650.1 & 20.83 & 6 & 13 & 1 & 0 \\
\hline 0124930201 & Medium & $215852.9-301328.8$ & 36244.5 & 20.23 & 8 & 13 & 0 & 0 \\
\hline 0130920101 & Thick & $220309.3+185227.9$ & 16490.5 & 20.78 & 1 & 13 & 1 & 0 \\
\hline 0012440301 & Thin1 & $220510.1-015511.2$ & 30935.9 & 20.79 & 2 & 13 & 2 & 1 \\
\hline 0106660101 & Thin1 & $221531.9-174402.6$ & 56908.9 & 20.37 & 1 & 13 & 1 & 0 \\
\hline 0009650201 & Medium & $221755.4-082058.0$ & 21026.5 & 20.73 & 8 & 13 & 4 & 0 \\
\hline 0103861201 & Medium & $221919.2+120800.1$ & 12172.6 & 20.73 & 8 & 13 & 1 & 0 \\
\hline
\end{tabular}


Table 2. continued.

\begin{tabular}{|c|c|c|c|c|c|c|c|c|}
\hline Obs. ID & Filter & RA; Dec (J2000) & $\begin{array}{r}\text { Exposure } \\
\mathrm{s} \\
(4)\end{array}$ & $\begin{array}{l}\log N h \\
\mathrm{~cm}^{-2} \\
(5)\end{array}$ & $\begin{array}{l}R_{\text {in }} \\
\operatorname{arcmin} \\
(6)\end{array}$ & $\begin{array}{l}R_{\text {out }} \\
\text { arcmin } \\
\text { (7) }\end{array}$ & $\begin{array}{l}\text { BSS } \\
\text { srcs } \\
(8)\end{array}$ & $\begin{array}{l}\text { HBSS } \\
\text { sres } \\
(9)\end{array}$ \\
\hline 0049340201 & Medium & $222045.1-244058.1$ & 26840.9 & 20.29 & 6 & 13 & 0 & 0 \\
\hline 0100440101 & Thick & $222830.4-051849.5$ & 45013.1 & 20.72 & 2 & 13 & 1 & 0 \\
\hline 0018741701 & Thin1 & $223432.9-374348.1$ & 7080.0 & 20.09 & 4 & 13 & 0 & 0 \\
\hline 0111790101 & Medium & $223545.9-260300.4$ & 41302.3 & 20.16 & 4 & 13 & 2 & 0 \\
\hline 0103860201 & Medium & $223655.9-221310.1$ & 8489.4 & 20.33 & 8 & 13 & 0 & 0 \\
\hline $0103860301^{*}$ & Medium & $224018.1+080323.2$ & 5879.2 & 20.82 & 8 & 13 & 1 & - \\
\hline 0006810101 & Medium & $224239.5+294335.6$ & 7009.1 & 20.81 & 8 & 13 & 0 & 0 \\
\hline 0109070401 & Medium & $224841.5-510957.9$ & 14721.9 & 20.13 & 8 & 13 & 2 & 0 \\
\hline 0112240101 & Medium & $224948.3-642311.2$ & 30606.8 & 20.47 & 8 & 13 & 3 & 0 \\
\hline 0081340901 & Medium & $225149.4-175217.0$ & 22397.4 & 20.43 & 1 & 13 & 1 & 0 \\
\hline 0112910301* & Thin1 & $225358.8-173355.8$ & 5301.0 & 20.43 & 8 & 13 & 1 & - \\
\hline 0009650101 & Medium & $225518.1-031035.6$ & 33943.5 & 20.66 & 8 & 13 & 0 & 0 \\
\hline 0112170301 & Medium & $230315.8+085225.9$ & 23348.5 & 20.69 & 4 & 13 & 0 & 0 \\
\hline 0109130701 & Thin1 & $230443.6-084114.5$ & 10615.1 & 20.56 & 8 & 13 & 1 & 0 \\
\hline 0033541001 & Thin1 & $230445.0+031135.6$ & 12460.9 & 20.72 & 2 & 13 & 2 & 0 \\
\hline 0025541001 & Medium & $230456.8+121932.0$ & 9954.8 & 20.70 & 3 & 13 & 5 & 0 \\
\hline 0123900101 & Thin1 & $231358.9-424328.3$ & 37399.4 & 20.25 & 6 & 13 & 1 & 0 \\
\hline $0109463601^{*}$ & Thin1 & $231518.7-591031.7$ & 5403.3 & 20.46 & 8 & 13 & 1 & - \\
\hline 0093640701 & Medium & $231611.0-423501.8$ & 14442.9 & 20.27 & 3 & 13 & 4 & 0 \\
\hline 0112880301 & Thick & $233149.9+195628.5$ & 14317.3 & 20.63 & 3 & 13 & 0 & 0 \\
\hline 0093550401 & Medium & $233340.0-151712.2$ & 22256.9 & 20.30 & 1 & 13 & 2 & 0 \\
\hline 0100241001 & Thick & $234940.6+362530.6$ & 8738.5 & 20.91 & 3 & 13 & 2 & 0 \\
\hline
\end{tabular}

Columns are as follows: (1) XMM-Newton Observation number; (2) Filter used; (3) Right ascension and Declination (J2000) of the MOS2 image center; (4) On-Axis good-time exposure; (5) Logarithm of the Galactic hydrogen column density along the line of sight from Dickey \& Lockman (1990); (6) Inner radius of the part of the MOS2 image used; (7) Outer radius of the part of the MOS2 image used; (8) total number of BSS sources found in the surveyed area of each MOS2 image; (9) total number of HBSS sources found in the surveyed area of each MOS2 image. See Sect. 2.4 for details.

NOTE $-{ }^{*}$ These 26 fields have not be considered for the definition of the HBSS sample. 
R. Della Ceca et al.: The XMM-Newton bright serendipitous survey, Online Material $p$

Table 3. Basic information on the XMM-Newton BSS sample.

\begin{tabular}{|c|c|c|c|c|c|c|c|}
\hline $\begin{array}{l}\text { Name } \\
\text { XBS... } \\
(1)\end{array}$ & Obs. ID & RA; Dec (J2000) & $\begin{array}{r}\text { OffAxis } \\
\operatorname{arcmin} \\
(4)\end{array}$ & $\begin{array}{r}\text { Rate } \\
\times 10^{-2} \mathrm{cts} / \mathrm{s} \\
(5)\end{array}$ & $H R 2$ & $H R 3$ & Class \\
\hline J000027.7-250442 & 0125310101 & $000027.8-250442.6$ & 2.10 & $1.13 \pm 0.08$ & $-0.48_{-0.06}^{+0.06}$ & $-0.37_{-0.11}^{+0.11}$ & AGN1 \\
\hline J000031.7-245502 & 0125310101 & $000031.8-245502.9$ & 11.68 & $1.41 \pm 0.13$ & $-0.67_{-0.06}^{+0.07}$ & $-0.37_{-017}^{+0.17}$ & AGN1 \\
\hline $\mathrm{J} 000100.2-250501^{d}$ & 0125310101 & $000100.2-250501.2$ & 6.97 & $1.38 \pm 0.10$ & $-0.37_{-0.07}^{+0.00}$ & $-0.47_{-0.10}^{+0.11}$ & AGN1 \\
\hline J000102.4-245850 & 0125310101 & $000102.5-245850.7$ & 10.72 & $1.48 \pm 0.12$ & $\begin{array}{l}-0.54_{-0.07}^{+0.07} \\
-0.07\end{array}$ & $\begin{array}{l}-0.71_{-0.10}^{-0.10} \\
-0.11\end{array}$ & AGN1 \\
\hline J000532.7+200716 & 0101040101 & $000532.8+200716.2$ & 12.15 & $2.47 \pm 0.12$ & $-0.81_{-0.03}^{+0.03}$ & $-0.70_{-0.10}^{+0.10}$ & GAL \\
\hline $\mathrm{J} 001002.4+110831$ & 0127110201 & $001002.4+110831.0$ & 12.12 & $13.12 \pm 0.71$ & $-0.79_{-0.03}^{+0.03}$ & $-0.88_{-0.06}^{+0.07}$ & star \\
\hline J001051.6+105140 & 0127110201 & $001051.6+105140.2$ & 8.61 & $1.01 \pm 0.15$ & $-0.76_{-0.10}^{+0.09}$ & $-1.00_{-0.00}^{+0.70}$ & star \\
\hline J001749.7+161952 & 0111000101 & $001749.7+161952.5$ & 12.11 & $3.35 \pm 0.15$ & $-1.00_{-0.00}^{+0.01}$ & $1.00_{-0.06}^{+0.00}$ & star \\
\hline J001831.6+162925 & 0111000101 & $001831.7+162925.6$ & 3.31 & $1.82 \pm 0.08$ & $-0.67_{-0.03}^{+0.00}$ & $-0.56_{-0.08}^{+0.08}$ & AGN1 \\
\hline $\mathrm{J} 002618.5+105019^{a}$ & 0001930101 & $002618.5+105019.3$ & 9.56 & $5.19 \pm 0.24$ & $-0.53_{-0.04}^{+0.03}$ & $-0.67_{-0.06}^{+0.06}$ & AGN1 \\
\hline J002637.4+165953 & 0050140201 & $002637.4+165953.5$ & 9.74 & $2.78 \pm 0.10$ & $-0.61_{-0.03}^{+0.04}$ & $-0.62^{-0.06}$ & AGN1 \\
\hline J002707.5+170748 & 0050140201 & $002707.6+170748.5$ & 7.78 & $1.58 \pm 0.08$ & $-0.58_{-0.04}^{+0.03}$ & $-0.39_{-0.08}^{+0.08}$ & AGN1 \\
\hline J002953.1+044524 & 0112320101 & $002953.2+044524.6$ & 10.69 & $1.33 \pm 0.12$ & $-0.91_{-0.04}^{+0.04}$ & $-1.00_{-0.00}^{+0.60}$ & star \\
\hline $\mathrm{J} 003255.9+394619^{d}$ & 0065770101 & $003255.9+394619.9$ & 11.90 & $1.11 \pm 0.21$ & $-0.76_{-0.11}^{+0.12}$ & $-0.24_{-0.44}^{+0.00}$ & AGN1 \\
\hline J003315.5-120700 & 0125920201 & $003315.6-120700.9$ & 9.52 & $1.62 \pm 0.20$ & $-0.75_{-0.08}^{+0.08}$ & $-0.05_{-0.26}^{+0.44}$ & AGN1 \\
\hline J003316.0-120456 & 0125920201 & $003316.1-120456.6$ & 9.66 & $1.03 \pm 0.15$ & $-0.90_{-0.06}^{+0.06}$ & $-0.97_{-0.03}^{+1.20}$ & AGN1 \\
\hline J003418.9-115940 & 0125920201 & $003419.0-115940.1$ & 9.60 & $1.05 \pm 0.14$ & $-0.69_{-0.09}^{+0.06}$ & $-0.39_{-0.26}^{+0.03}$ & AGN1 \\
\hline J005009.9-515934 & 0125320701 & $005009.9-515934.7$ & 8.10 & $1.38 \pm 0.11$ & $\begin{array}{r}-0.09 \\
-0.56_{-0.07}^{+0.07}\end{array}$ & $-0.57_{-0.13}^{-0.26}$ & AGN1 \\
\hline J005031.1-520012 & 0125320701 & $005031.1-520012.8$ & 8.57 & $1.89 \pm 0.14$ & $-0.65_{-0.05}^{+0.05}$ & $-0.47_{-0.13}^{+0.13}$ & AGN1 \\
\hline J005032.3-521543 & 0125320701 & $005032.3-521543.5$ & 9.28 & $1.12 \pm 0.13$ & $-0.45_{-0.09}^{+0.09}$ & $-0.53_{-0.15}^{+0.13}$ & AGN1 \\
\hline J005822.9-274016 & 0111280301 & $005822.9-274016.1$ & 7.71 & $2.07 \pm 0.21$ & $-0.91_{-0.05}^{+0.09}$ & $-0.19_{-0.48}^{+0.13}$ & star \\
\hline J010421.4-061418 & 0112650401 & $010421.4-061418.8$ & & $1.01 \pm 0.09$ & $\begin{array}{r}-0.05 \\
-0.49_{-0.08}^{+0.08}\end{array}$ & $-0.81_{-0.11}^{+0.38}$ & AGN1 \\
\hline J010432.8-583712 & 0103861601 & $010432.8-583712.2$ & 12.41 & $1.61 \pm 0.26$ & $-0.53_{-0.13}^{+0.14}$ & $-0.07_{-0.27}^{+0.26}$ & AGN1 \\
\hline J010701.5-172748 & 0025540101 & $010701.5-172748.4$ & 10.99 & $3.56 \pm 0.33$ & $-0.47_{-0.08}^{-0.13}$ & $-0.84_{-0.09}^{+0.27}$ & AGN1 \\
\hline J010747.2-172044 & 0025540101 & $010747.3-172044.6$ & 9.49 & $2.27 \pm 0.23$ & $-0.62_{-0.08}^{+0.08}$ & $-0.48_{-0.17}^{+0.09}$ & AGN1 \\
\hline J012000.0-110429 & 0113040801 & $012000.1-110429.3$ & 12.66 & $1.18 \pm 0.25$ & $-0.77_{-0.14}^{+0.08}$ & $0.01_{-0.53}^{+0.17}$ & AGN1 \\
\hline J012025.2-105441 & 0113040801 & $012025.3-105441.6$ & 3.95 & $1.39 \pm 0.18$ & $-0.50_{-0.11}^{+0.14}$ & $-0.68_{-0.18}^{+0.16}$ & \\
\hline J012057.4-110444 & 0113040801 & $012057.5-110444.6$ & 9.40 & $2.34 \pm 0.28$ & $-0.61_{-0.09}^{+0.09}$ & $-0.48_{-0.21}^{+0.18}$ & AGN2 \\
\hline J012119.9-110418 & 0113040801 & $012120.0-110418.6$ & 12.65 & $5.85 \pm 0.48$ & $-0.71_{-0.06}^{+0.06}$ & $-0.67_{-0.16}^{+0.15}$ & AGN1 \\
\hline $\mathrm{J} 012505.4+014624^{d}$ & 0109860101 & $012505.4+014624.4$ & 7.04 & $1.94 \pm 0.09$ & $-0.46_{-0.04}^{+0.00}$ & $-0.51_{-0.06}^{+0.10}$ & AGN1 \\
\hline $\mathrm{J} 012540.2+015752$ & 0109860101 & $012540.3+015752.1$ & 12.35 & $1.41 \pm 0.10$ & $-0.56_{-0.06}^{+0.04}$ & $\begin{array}{r}-0.06 \\
-0.29_{-0.12}^{+0.11}\end{array}$ & AGN2 \\
\hline $\mathrm{J} 012654.3+191246^{d}$ & 0112600601 & $012654.3+191247.0$ & 9.20 & $1.02 \pm 0.26$ & $-0.64_{-0.17}^{+0.17}$ & $0.07_{-0.39}^{+0.41}$ & GAL \\
\hline J012757.3+185923 & 0112600601 & $012757.4+185923.9$ & 12.64 & $34.37 \pm 1.49$ & $-0.92_{-0.02}^{-0.02}$ & $-0.34_{-0.16}^{+0.39}$ & star \\
\hline $\mathrm{J} 012757.2+190000$ & 112600601 & $012757.2+190000.4$ & 12.09 & $10.69 \pm 0.81$ & $-0.81_{-0.04}^{+0.02}$ & $-1.00_{-0.00}^{+0.10}$ & star \\
\hline J013204.9-400050 & 112630201 & $013204.9-400050.9$ & 12.23 & $1.29 \pm 0.12$ & $\begin{array}{r}-0.04 \\
-0.67_{-0.06}^{+0.06}\end{array}$ & $-0.32_{-0.16}^{+0.16}$ & AGN1 \\
\hline $\mathrm{J} 013240.1-133307^{a, d}$ & 0084230301 & $013240.1-133307.8$ & 11. & $1.42 \pm 0.14$ & $-0.02_{-0.10}^{+0.06}$ & $\begin{array}{r}-0.16 \\
-0.37_{-0.11}^{+0.11}\end{array}$ & AGN2 \\
\hline $\mathrm{J} 013811.7-175416^{d}$ & 0111430101 & $013811.8-175416.3$ & 12.09 & $3.09 \pm 0.17$ & $-0.73_{-0.04}^{+0.04}$ & $-0.77_{-0.08}^{+0.08}$ & BL? \\
\hline J013924.8-674126 & 0032140401 & $013924.8-674126.5$ & 8.50 & $1.02 \pm 0.14$ & $0.21_{-0.14}^{+0.14}$ & $-0.54_{-0.14}^{+0.00}$ & \\
\hline J013944.0-674909 ${ }^{a}$ & 0032140401 & $013944.0-674909.4$ & 2.69 & $2.04 \pm 0.17$ & $-0.50_{-0.07}^{+0.14}$ & $-0.43_{-0.13}^{+0.14}$ & AGN1? \\
\hline $\mathrm{J} 014100.6-675328^{a}$ & 0032140401 & $014100.7-675329.0$ & 6.64 & $57.52 \pm 1.03$ & $-0.45_{-0.02}^{+0.07}$ & $-0.38_{-0.03}^{+0.03}$ & star \\
\hline J014109.9-675639 & 0032140401 & $014109.9-675639.6$ & 9.67 & $1.97 \pm 0.23$ & $-0.35_{-0.10}^{+0.11}$ & $-0.45_{-0.15}^{+0.16}$ & AGN1? \\
\hline $\mathrm{J} 014227.0+133453$ & 0093641001 & $014227.1+133453.7$ & 9.12 & $1.29 \pm 0.14$ & $-0.52_{-0.10}^{+0.10}$ & $-0.51_{-0.18}^{+0.15}$ & AGN2? \\
\hline J014251.5+133352 & 0093641001 & $014251.6+133352.8$ & 5.15 & $1.43 \pm 0.13$ & $-0.46_{-0.08}^{+0.09}$ & $-0.49_{-0.14}^{+0.18}$ & AGN1 \\
\hline $\mathrm{J} 015916.9+003010^{c}$ & 101640201 & $015917.0+003010.4$ & 10.49 & $5.74 \pm 0.54$ & $-0.58_{-0.06}^{+0.08}$ & $-0.71_{-0.20}^{+0.14}$ & $\mathrm{CL}+\mathrm{BL} ?$ \\
\hline $\mathrm{J} 015957.5+003309^{a}$ & 0101640201 & $015957.5+003309.7$ & 9.56 & $6.50 \pm 0.39$ & -0.6 & $-0.51_{-0.10}^{+0.20}$ & AGN1 \\
\hline J020029.0+002846 & 0101640201 & $020029.0+002846.1$ & 10.90 & $3.39 \pm 0.30$ & $-0.62_{-0.07}^{-0.00}$ & $-0.36_{-0.15}^{+0.10}$ & AGN1 \\
\hline J020757.3+351828 & 0084140101 & $020757.3+351828.9$ & 9.48 & $1.19 \pm 0.07$ & $-0.52_{-0.05}^{+0.05}$ & $-0.55_{-0.09}^{+0.09}$ & AGN1 \\
\hline J020845.1+351438 & 0084140101 & $020845.2+351438.0$ & 8.49 & $1.80 \pm 0.09$ & $-0.48_{-0.04}^{+0.04}$ & $-0.52_{-0.07}^{+0.07}$ & AGN1 \\
\hline J020916.4+351850 & 0084140101 & $020916.5+351850.4$ & 8.85 & $1.30 \pm 0.08$ & $-0.49_{-0.05}^{+0.04}$ & $\begin{array}{r}-0.07 \\
-0.66_{-0.08}^{+0.08}\end{array}$ & \\
\hline $\mathrm{J} 021640.7-044404^{a, d}$ & 0112371701 & $021640.7-044404.9$ & 9.35 & $2.56 \pm 0.17$ & $-0.72_{-0.05}^{+0.05}$ & $-0.26_{-0.13}^{+0.08}$ & AGN1 \\
\hline J021642.3-043553 & 0112371701 & $021642.4-043553.0$ & 8.14 & $1.32 \pm 0.12$ & $\begin{array}{r}-0.05 \\
-0.42_{-0.08}^{+0.08}\end{array}$ & $-0.61_{-0.11}^{+0.13}$ & \\
\hline $\mathrm{J} 021808.3-045845^{a}$ & 0112371001 & $021808.3-045845.7$ & 2.26 & $4.87 \pm 0.10$ & $-0.65_{-0.02}^{+0.08}$ & $-0.52_{-0.04}^{+0.04}$ & AGN1 \\
\hline $\mathrm{J} 021817.4-045113^{a}$ & 0112371001 & $021817.4-045113.3$ & 9.58 & $4.47 \pm 0.13$ & $-0.48_{-0.03}^{+0.02}$ & $-0.56_{-0.04}^{+0.04}$ & AGN1 \\
\hline
\end{tabular}


Table 3. continued.

\begin{tabular}{|c|c|c|c|c|c|c|c|}
\hline $\begin{array}{l}\text { Name } \\
\text { XBS... } \\
(1)\end{array}$ & Obs. ID & $\overline{\text { RA; Dec (J2000) }}$ & $\begin{array}{r}\text { OffAxis } \\
\text { arcmin } \\
(4)\end{array}$ & $\begin{array}{r}\text { Rate } \\
\times 10^{-2} \mathrm{cts} / \mathrm{s} \\
(5)\end{array}$ & $H R 2$ & $\overline{H R 3}$ & Class \\
\hline J021820.6-050427 & 0112371001 & $021820.7-050427.6$ & 6.90 & $1.22 \pm 0.06$ & $-0.47_{-0.04}^{+0.04}$ & $-0.57_{-0.07}^{+0.07}$ & AGN1 \\
\hline J021830.0-045514 & 0112371001 & $021830.0-045514.8$ & 8.69 & $3.36 \pm 0.10$ & $-0.90_{-0.01}^{+0.04}$ & $-0.69_{-0.09}^{+0.09}$ & star \\
\hline J021923.2-045148 & 0112370301 & $021923.2-045148.1$ & 8.63 & $1.40 \pm 0.07$ & $-0.66_{-0.04}^{+0.04}$ & $-0.42_{-0.10}^{+0.09}$ & AGN1 \\
\hline J022253.0-044515 & 0109520501 & $022253.0-044516.0$ & 8.12 & $1.15 \pm 0.09$ & $-0.58_{-0.07}^{+0.04}$ & $-0.60_{-0.13}^{+0.10}$ & \\
\hline J022339.6-050728 & 0111110401 & $022339.6-050728.2$ & 5.66 & $1.45 \pm 0.08$ & $-0.53_{-0.05}^{+0.05}$ & $-0.76_{-0.07}^{+0.07}$ & \\
\hline J022707.7-050819 & 0111110201 & $022707.7-050819.8$ & 7.05 & $1.89 \pm 0.19$ & $0.17_{-0.09}^{+0.09}$ & $-0.67_{-0.08}^{+0.08}$ & AGN2 \\
\hline J023459.7-294436 & 0103861101 & $023459.7-294436.9$ & 8.91 & $1.18 \pm 0.23$ & $-0.26_{-0.14}^{+0.14}$ & $-0.48_{-0.19}^{+0.18}$ & \\
\hline J023530.2-523045 & 0098810101 & $023530.2-523045.2$ & 12.59 & $1.88 \pm 0.14$ & $-0.70_{-0.05}^{+0.05}$ & $-0.38_{-0.14}^{+0.14}$ & AGN1 \\
\hline $\mathrm{J} 023713.5-522734^{a}$ & 0098810101 & $023713.5-522734.4$ & 12.07 & $5.60 \pm 0.24$ & $-0.57_{-0.03}^{-0.05}$ & $-0.58_{-0.06}^{+0.07}$ & AGN1 \\
\hline J023853.2-521911 & 0067190101 & $023853.3-521911.1$ & 9.22 & $1.45 \pm 0.11$ & $-0.49_{-0.06}^{+0.06}$ & $-0.42_{-0.11}^{+0.11}$ & AGN1 \\
\hline J024200.9+000020 & 0111200101 & $024201.0+000020.1$ & 10.11 & $2.13 \pm 0.12$ & $-0.60_{-0.04}^{+0.00}$ & $-0.52_{-0.09}^{+0.09}$ & AGN1 \\
\hline J024204.7+000814 & 0111200101 & $024204.7+000814.3$ & 12.90 & $1.01 \pm 0.10$ & $-0.57_{-0.07}^{+0.08}$ & $-0.81_{-0.10}^{+0.10}$ & AGN1 \\
\hline J024207.3+000037 & 0111200101 & $024207.3+000037.6$ & 8.59 & $1.26 \pm 0.09$ & $-0.62_{-0.05}^{+0.05}$ & $-0.77_{-0.09}^{+0.09}$ & AGN1 \\
\hline J024325.6-000413 & 0111200101 & $024325.6-000413.3$ & 11.61 & $1.04 \pm 0.08$ & $-0.31_{-0.07}^{+0.07}$ & $-0.40_{-0.10}^{+0.10}$ & AGN1 \\
\hline $\mathrm{J} 025606.1+001635$ & 0056020301 & $025606.2+001635.5$ & 12.49 & $1.28 \pm 0.15$ & $-0.45_{-0.09}^{+0.09}$ & $-0.93_{-0.06}^{+0.06}$ & AGN1 \\
\hline J025645.4+000031 & 0056020301 & $025645.5+000031.3$ & 6.35 & $1.01 \pm 0.10$ & $-0.49_{-0.08}^{+0.08}$ & $-0.65_{-0.13}^{+0.06}$ & AGN1? \\
\hline J030204.2+001625 & 0041170101 & $030204.2+001625.6$ & 12.36 & $1.01 \pm 0.07$ & $-0.46_{-0.06}^{+0.06}$ & $-0.50_{-0.10}^{+0.10}$ & \\
\hline $\mathrm{J} 030206.8-000121^{a}$ & 0041170101 & $030206.9-000121.2$ & 11.90 & $3.56 \pm 0.13$ & $-0.53_{-0.03}^{+0.06}$ & $-0.46_{-0.06}^{+0.10}$ & AGN1 \\
\hline $\mathrm{J} 030614.1-284019^{a}$ & 0042340501 & $030614.2-284019.9$ & 10.87 & $3.85 \pm 0.23$ & $-0.49_{-0.05}^{+0.05}$ & $-0.35_{-0.09}^{+0.09}$ & AGN1 \\
\hline J030641.0-283559 & 0042340501 & $030641.0-283559.5$ & 6.66 & $1.25 \pm 0.11$ & $-0.61_{-0.07}^{+0.05}$ & $-0.81_{-0.11}^{+0.11}$ & AGN1 \\
\hline $\mathrm{J} 031015.5-765131^{a}$ & 0122520201 & $031015.6-765131.5$ & 5.90 & $5.36 \pm 0.15$ & $-0.46_{-0.03}^{+0.03}$ & $-0.53_{-0.04}^{+0.04}$ & AGN1 \\
\hline $\mathrm{J} 031146.1-550702^{a}$ & 0110970101 & $031146.1-550702.5$ & 12.38 & $6.55 \pm 0.45$ & $-0.58_{-0.05}^{+0.05}$ & $-0.39_{-0.11}^{+0.11}$ & AGN2 \\
\hline J031311.7-765428 & 0122520201 & $031311.7-765428.8$ & 4.86 & $1.10 \pm 0.07$ & $-0.43_{-0.06}^{+0.05}$ & $\begin{array}{r}-0.11 \\
-0.30_{-0.09}^{+0.09}\end{array}$ & AGN1 \\
\hline J031401.3-545959 & 0110970101 & $031401.3-545959.5$ & 8.34 & $1.29 \pm 0.14$ & $-0.51_{-0.09}^{+0.09}$ & $-0.68_{-0.14}^{+0.14}$ & AGN1 \\
\hline J031549.4-551811 & 0129320901 & $031549.4-551811.5$ & 12.22 & $1.04 \pm 0.16$ & $-0.83_{-0.08}^{+0.09}$ & $-0.85_{-0.13}^{+0.21}$ & AGN1 \\
\hline J031851.9-441815 & 0105660101 & $031851.9-441815.3$ & 10.78 & $1.15 \pm 0.12$ & $-0.59_{-0.07}^{+0.08}$ & $-0.40_{-0.15}^{+0.16}$ & AGN1 \\
\hline $\mathrm{J} 031859.2-441627^{a, b}$ & 0105660101 & $031859.3-441627.6$ & 11.55 & $1.54 \pm 0.14$ & $-0.21_{-0.08}^{+0.07}$ & $-0.47_{-0.10}^{+0.15}$ & AGN1 \\
\hline J033208.7-274735 & 0108060501 & $033208.7-274735.7$ & 4.60 & $1.13 \pm 0.06$ & $-0.70_{-0.04}^{+0.03}$ & $-0.40_{-0.09}^{+0.10}$ & AGN2? \\
\hline $\mathrm{J} 033226.9-274107^{d}$ & 0108060501 & $033227.0-274107.3$ & 7.35 & $1.42 \pm 0.07$ & $-0.63_{-0.04}^{+0.04}$ & $-0.59_{-0.07}^{+0.09}$ & AGN1 \\
\hline J033435.5-254259 & 0099010101 & $033435.6-254259.3$ & 11.87 & $1.10 \pm 0.12$ & $-0.50_{-0.09}^{+0.09}$ & $-1.00_{-0.00}^{+0.18}$ & AGN1 \\
\hline J033453.9-254154 & 0099010101 & $033454.0-254154.6$ & 8.14 & $1.89 \pm 0.13$ & $-0.62_{-0.05}^{+0.05}$ & $-0.38_{-0.12}^{+0.12}$ & AGN1 \\
\hline J033506.0-255619 & 0099010101 & $033506.1-255619.9$ & 12.41 & $1.18 \pm 0.12$ & $\begin{array}{l}-0.05 \\
-0.03_{-0.08}^{+0.08}\end{array}$ & $-0.82_{-0.16}^{+0.12}$ & AGN1 \\
\hline J033851.4-352646 & 0055140101 & $033851.5-352646.3$ & 8.89 & $1.38 \pm 0.08$ & $-0.55_{-0.04}^{+0.04}$ & $-0.68_{-0.07}^{+0.07}$ & AGN1 \\
\hline J033912.1-352813 & 0055140101 & $033912.1-352813.8$ & 5.18 & $1.23 \pm 0.06$ & $-0.40_{-0.04}^{+0.04}$ & $-0.52_{-0.06}^{+0.07}$ & \\
\hline J033942.8-352411 & 0055140101 & $033942.8-352411.3$ & 2.40 & $1.33 \pm 0.06$ & $-0.75_{-0.03}^{+0.03}$ & $-0.56_{-0.09}^{+0.09}$ & AGN1 \\
\hline J040744.6-710846 & 0111970301 & $040744.6-710847.0$ & 11.15 & $1.27 \pm 0.15$ & $-0.77_{-0.07}^{+0.03}$ & $-0.75_{-0.22}^{+0.18}$ & \\
\hline J040807.2-712702 & 0111970301 & $040807.2-712702.5$ & 10.47 & $6.46 \pm 0.27$ & $-0.91_{-0.02}^{+0.02}$ & $-0.74_{-0.11}^{+0.12}$ & star \\
\hline $\mathrm{J} 041108.1-711341^{a}$ & 0111970301 & $041108.1-711341.1$ & 10.52 & $1.03 \pm 0.11$ & $-0.53_{-0.08}^{+0.02}$ & $-0.04_{-0.16}^{+0.16}$ & AGN1 \\
\hline J042417.9-571539 & 0112600401 & $042417.9-571539.2$ & 11.86 & $1.04 \pm 0.18$ & $-0.64_{-0.13}^{+0.00}$ & $-0.46_{-0.30}^{+0.30}$ & \\
\hline J043448.3-775329 & 0103861701 & $043448.4-775329.2$ & 8.55 & $2.03 \pm 0.22$ & $-0.35_{-0.10}^{+0.13}$ & $-0.61_{-0.13}^{+0.14}$ & \\
\hline $\mathrm{J} 045942.4+015843$ & 0112880401 & $045942.5+015843.5$ & 11.59 & $1.38 \pm 0.13$ & $\begin{array}{r}-0.10 \\
-0.35_{-0.08}^{+0.09}\end{array}$ & $-0.49_{-0.12}^{+0.13}$ & AGN1 \\
\hline J050011.7+013948 & 0112880401 & $050011.8+013948.0$ & 11.76 & $1.19 \pm 0.11$ & $-0.35_{-0.09}^{+0.09}$ & $-0.38_{-0.13}^{+0.12}$ & AGN1? \\
\hline J050446.3-283821 & 0111160201 & $050446.3-283821.8$ & 12.92 & $1.36 \pm 0.10$ & $-0.57_{-0.05}^{-0.09}$ & $-0.67_{-0.10}^{+0.13}$ & \\
\hline J050453.4-284532 & 0111160201 & $050453.5-284532.1$ & 6.75 & $1.06 \pm 0.06$ & $-0.39_{-0.05}^{+0.05}$ & $-0.41_{-0.08}^{+0.08}$ & \\
\hline J050501.8-284149 & 0111160201 & $050501.9-284149.1$ & 8.18 & $1.41 \pm 0.08$ & $-0.65_{-0.04}^{+0.05}$ & $\begin{array}{r}-0.51_{-0.09}^{+0.09} \\
-0.58\end{array}$ & \\
\hline J050536.6-290050 & 0111160201 & $050536.7-290050.8$ & 12.43 & $1.68 \pm 0.10$ & $-0.27_{-0.05}^{+0.04}$ & $-0.47_{-0.07}^{+0.07}$ & \\
\hline J051413.5+794345 & 0094400101 & $051413.6+794345.8$ & 7.42 & $1.43 \pm 0.09$ & $-0.29_{-0.06}^{+0.06}$ & $-0.53_{-0.08}^{+0.08}$ & \\
\hline J051617.1+794408 & 0094400101 & $051617.2+794408.7$ & 4.00 & $4.05 \pm 0.12$ & $-0.85_{-0.02}^{+0.02}$ & $-0.73_{-0.07}^{+0.08}$ & star \\
\hline J051651.9+794314 & 0094400101 & $051651.9+794314.6$ & 3.01 & $1.77 \pm 0.08$ & $-0.28_{-0.04}^{+0.04}$ & $-0.55_{-0.05}^{+0.06}$ & \\
\hline J051655.3-104104 & 0103860701 & $051655.3-104104.0$ & 11.23 & $1.97 \pm 0.31$ & $-0.33_{-0.14}^{+0.04}$ & $\begin{array}{r}-0.05 \\
-1.00_{-0.00}^{+0.29}\end{array}$ & AGN1 \\
\hline J051822.6+793208 & 0094400101 & $051822.7+793208.0$ & 9.37 & $1.27 \pm 0.09$ & $-0.54_{-0.06}^{+0.14}$ & $-0.41_{-0.11}^{+0.10}$ & GAL \\
\hline J051955.5-455727 & 0090050701 & $051955.5-455727.4$ & 10.69 & $3.27 \pm 0.24$ & $-0.51_{-0.06}^{+0.06}$ & $\begin{array}{r}-0.77_{-0.08}^{+0.11} \\
-0.08\end{array}$ & \\
\hline J052022.0-252309 & 0085640101 & $052022.0-252309.5$ & 8.87 & $1.21 \pm 0.14$ & $-0.66_{-0.09}^{+0.09}$ & $-0.37_{-0.22}^{+0.21}$ & \\
\hline J052048.9-454128 & 0090050701 & $052048.9-454128.5$ & 11.67 & $8.35 \pm 0.45$ & $-0.95_{-0.02}^{+0.02}$ & $-0.81_{-0.17}^{+0.17}$ & star \\
\hline $\mathrm{J} 052108.5-251913^{d}$ & 0085640101 & $052108.5-251913.1$ & 3.17 & $3.54 \pm 0.20$ & $-0.59_{-0.05}^{+0.02}$ & $-0.55_{-0.09}^{+0.09}$ & AGN1 \\
\hline J052116.2-252957 & 0085640101 & $0521 \quad 16.2-252957.8$ & 8.80 & $1.00 \pm 0.15$ & $-0.50_{-0.12}^{+0.11}$ & $-0.70_{-0.17}^{+0.18}$ & \\
\hline
\end{tabular}


Table 3. continued.

\begin{tabular}{|c|c|c|c|c|c|c|c|}
\hline $\begin{array}{l}\text { Name } \\
\text { XBS... } \\
(1)\end{array}$ & Obs. ID & RA; Dec (J2000) & $\begin{array}{r}\text { OffAxis } \\
\operatorname{arcmin} \\
(4)\end{array}$ & $\begin{array}{r}\text { Rate } \\
\times 10^{-2} \mathrm{cts} / \mathrm{s} \\
(5)\end{array}$ & $H R 2$ & $H R 3$ & Class \\
\hline J052144.1-251518 & 0085640101 & $052144.1-251518.8$ & 11.77 & $1.25 \pm 0.19$ & $-0.60_{-0.12}^{+0.11}$ & $-0.87_{-0.12}^{+0.17}$ & \\
\hline J052155.0-252200 & 0085640101 & $052155.0-252200.7$ & 12.24 & $1.82 \pm 0.24$ & $-1.00_{-0.00}^{+0.07}$ & - & star \\
\hline J052509.3-333051 & 0050150101 & $052509.3-333051.9$ & 12.38 & $1.38 \pm 0.18$ & $-0.56_{-0.10}^{+0.10}$ & $-0.95_{-0.05}^{+0.09}$ & \\
\hline J052543.6-334856 & 0050150101 & $052543.6-334856.4$ & 9.70 & $1.01 \pm 0.15$ & $-0.53_{-0.10}^{+0.11}$ & $-0.76_{-0.14}^{+0.05}$ & \\
\hline $\mathrm{J} 061342.7+710725^{d}$ & 0009220601 & $061342.8+710725.9$ & 10.51 & $6.44 \pm 0.34$ & $-0.67_{-0.04}^{+0.04}$ & $-0.75_{-0.07}^{+0.08}$ & $\mathrm{BL}$ \\
\hline J062134.8-643150 & 0103860101 & $062134.8-643150.5$ & 11.02 & $1.04 \pm 0.20$ & $-0.41_{-0.16}^{+0.04}$ & $-0.22_{-0.25}^{+0.23}$ & \\
\hline J062425.7-642958 & 0103860101 & $062425.7-642958.3$ & 10.39 & $1.99 \pm 0.22$ & $-1.00_{-0.00}^{+0.04}$ & $1.00_{-1.30}^{+0.00}$ & star \\
\hline J063709.1+820949 & 0029340101 & $063709.2+820949.1$ & 10.46 & $1.28 \pm 0.11$ & $-0.58_{-0.06}^{+0.00}$ & $-0.52_{-0.13}^{+0.30}$ & \\
\hline J065214.1+743230 & 0061540101 & $065214.1+743230.6$ & 7.01 & $2.33 \pm 0.14$ & $-0.49_{-0.05}^{+0.05}$ & $-0.58_{-0.08}^{+0.08}$ & AGN1? \\
\hline J065237.4+742421 & 0061540101 & $065237.5+742421.9$ & 2.09 & $1.08 \pm 0.09$ & $-0.62_{-0.06}^{+0.05}$ & $-0.35_{-0.12}^{+0.12}$ & \\
\hline J065400.0+742045 & 0061540101 & $065400.1+742045.1$ & 8.72 & $1.82 \pm 0.15$ & $-0.49_{-0.06}^{+0.06}$ & $-0.65_{-0.10}^{+0.10}$ & AGN1 \\
\hline J065744.3-560817 & 0112980201 & $065744.3-560817.5$ & 11.74 & $1.40 \pm 0.10$ & $-0.56_{-0.05}^{+0.05}$ & $-0.78_{-0.08}^{+0.08}$ & \\
\hline J065839.5-560813 & 0112980201 & $065839.6-560813.3$ & 11.14 & $1.60 \pm 0.10$ & $-0.65_{-0.04}^{-0.05}$ & $-0.45_{-0.10}^{+0.10}$ & \\
\hline $\mathrm{J} 074202.7+742625$ & 0123100101 & $074202.7+742625.8$ & 10.86 & $3.02 \pm 0.13$ & $-0.59_{-0.03}^{+0.03}$ & $-0.28_{-0.07}^{+0.07}$ & AGN1 \\
\hline $\mathrm{J} 074312.1+742937$ & 0123100101 & $074312.1+742937.4$ & 5.33 & $19.59 \pm 0.25$ & $-0.61_{-0.01}^{+0.01}$ & $-0.56_{-0.02}^{+0.02}$ & AGN1 \\
\hline J074338.7+495431 & 0103862101 & $074338.7+495431.1$ & 12.22 & $1.27 \pm 0.23$ & $-0.14_{-0.20}^{+0.20}$ & $-0.47_{-0.24}^{+0.24}$ & AGN1? \\
\hline $\mathrm{J} 074352.0+744258$ & 0123100101 & $074352.1+744259.0$ & 9.30 & $1.99 \pm 0.10$ & $-0.56_{-0.04}^{+0.04}$ & $-0.49_{-0.08}^{+0.08}$ & AGN1 \\
\hline J074359.7+744057 & 0123100101 & $074359.7+744057.5$ & 7.25 & $1.47 \pm 0.08$ & $-0.85_{-0.03}^{+0.04}$ & $-0.62_{-0.14}^{+0.13}$ & star \\
\hline $\mathrm{J} 075117.9+180856^{b}$ & 0111100301 & $075117.9+180856.1$ & 2.37 & $1.65 \pm 0.08$ & $-0.35_{-0.04}^{+0.03}$ & $-0.46_{-0.06}^{+0.06}$ & AGN1? \\
\hline J080309.8+650807 & 0094400301 & $080309.8+650807.6$ & 12.10 & $2.91 \pm 0.22$ & $-0.97_{-0.01}^{+0.02}$ & $-1.00_{-0.00}^{+1.30}$ & star \\
\hline J080411.3+650906 & 0094400301 & $080411.4+650906.2$ & 9.49 & $1.39 \pm 0.12$ & $0.06_{-0.09}^{+0.09}$ & $-0.51_{-0.09}^{+0.09}$ & \\
\hline $\mathrm{J} 080504.6+245156$ & 0094530401 & $080504.6+245156.4$ & 7.23 & $1.07 \pm 0.13$ & $-0.53_{-0.11}^{+0.11}$ & $-0.56_{-0.20}^{+0.29}$ & \\
\hline $\mathrm{J} 080608.1+244420$ & 0094530401 & $080608.2+244420.4$ & 9.34 & $4.49 \pm 0.30$ & $-0.74_{-0.04}^{+0.01}$ & $-0.48_{-0.13}^{+0.14}$ & AGN1 \\
\hline J083049.8+524908 & 0092800101 & $083049.8+524908.2$ & 8.81 & $1.40 \pm 0.13$ & $-0.48_{-0.08}^{-0.04}$ & $-0.85_{-0.08}^{+0.09}$ & \\
\hline J083737.1+254751 & 0025540301 & $083737.2+254751.1$ & 10.61 & $9.08 \pm 0.37$ & $-0.47_{-0.04}^{+0.04}$ & $-0.53_{-0.06}^{+0.06}$ & AGN1 \\
\hline $\mathrm{J} 083737.0+255151$ & 0025540301 & $083737.1+255151.2$ & 12.25 & $2.89 \pm 0.23$ & $-0.34_{-0.08}^{+0.07}$ & $-0.52_{-0.10}^{+0.10}$ & AGN2 \\
\hline J083838.6+253616 & 0025540301 & $083838.6+253616.8$ & 9.63 & $1.32 \pm 0.16$ & $-0.57_{-0.09}^{+0.09}$ & $-0.45_{-0.19}^{+0.20}$ & AGN1 \\
\hline $\mathrm{J} 083905.9+255010$ & 0025540301 & $083905.9+255010.5$ & 10.89 & $1.02 \pm 0.15$ & $\begin{array}{r}-0.69_{-0.10}^{+0.10} \\
-0.00\end{array}$ & $-1.00_{-0.00}^{+0.54}$ & \\
\hline $\mathrm{J} 084026.2+650638^{d}$ & 0111400101 & $084026.2+650638.5$ & 9.56 & $1.21 \pm 0.09$ & $-0.50_{-0.07}^{+0.06}$ & $-0.27_{-0.12}^{+0.12}$ & AGN1 \\
\hline J084651.7+344634 & 0103660201 & $084651.7+344634.1$ & 10.51 & $1.05 \pm 0.15$ & $-0.71_{-0.08}^{+0.08}$ & $-1.00_{-0.00}^{+0.42}$ & AGN1 \\
\hline $\mathrm{J} 085427.8+584158$ & 0085030101 & $085427.8+584158.9$ & 9.06 & $2.92 \pm 0.32$ & $-0.83_{-0.06}^{+0.06}$ & $-0.71_{-0.27}^{+0.23}$ & star \\
\hline J085530.7+585129 & 0085030101 & $085530.8+585129.1$ & 7.42 & $1.21 \pm 0.19$ & $-0.64_{-0.11}^{+0.11}$ & $-1.00_{-0.00}^{+0.47}$ & \\
\hline J090729.1+620824 & 0110660201 & $090729.2+620824.6$ & 11.48 & $1.00 \pm 0.17$ & $-0.26_{-0.15}^{+0.11}$ & $-0.41_{-0.20}^{+0.20}$ & GAL \\
\hline J091043.4+054757 & 0083240201 & $091043.4+054757.8$ & 11.54 & $2.40 \pm 0.19$ & $-0.80_{-0.04}^{+0.04}$ & $-1.00_{-0.00}^{+0.25}$ & star \\
\hline J091828.4+513931 & 0084230601 & $091828.5+513931.3$ & 6.92 & $1.09 \pm 0.11$ & $0.31_{-0.10}^{+0.09}$ & $-0.39_{-0.09}^{+0.09}$ & AGN1 \\
\hline J094526.2-085006 & 0017540101 & $094526.2-085006.5$ & 11.06 & $1.39 \pm 0.20$ & $-0.50_{-0.12}^{+0.10}$ & $-0.80_{-0.18}^{+0.16}$ & \\
\hline J094548.3-084824 & 0017540101 & $094548.3-084824.6$ & 10.87 & $1.02 \pm 0.16$ & $-0.58_{-0.13}^{+0.12}$ & $-0.24_{-0.27}^{+0.18}$ & \\
\hline J095054.5+393924 & 0111290101 & $095054.5+393924.3$ & 12.46 & $1.19 \pm 0.13$ & $-0.59_{-0.09}^{+0.08}$ & $-0.32_{-0.18}^{+0.19}$ & AGN1 \\
\hline J095134.6-015451 & 0065790101 & $095134.6-015451.6$ & 10.87 & $1.05 \pm 0.16$ & $-0.50_{-0.13}^{+0.13}$ & $-0.72_{-0.25}^{+0.19}$ & \\
\hline J095218.9-013643 & 0065790101 & $095219.0-013643.1$ & 11.78 & $9.02 \pm 0.51$ & $-0.77_{-0.03}^{+0.03}$ & $0.41_{-0.09}^{+0.09}$ & AGN1 \\
\hline J095309.7+013558 & 0070940101 & $095309.8+013558.6$ & 8.00 & $1.15 \pm 0.19$ & $-0.64_{-0.13}^{+0.12}$ & $-0.54_{-0.43}^{+0.30}$ & \\
\hline $\mathrm{J} 095341.1+014204^{c}$ & 0070940101 & $095341.2+014204.4$ & 7.14 & $16.29 \pm 0.72$ & $-0.62_{-0.04}^{+0.04}$ & $-0.83_{-0.10}^{+0.09}$ & CL \\
\hline J095416.9+173627 & 0112850101 & $095416.9+173627.8$ & 11.71 & $4.01 \pm 0.23$ & $-0.72_{-0.04}^{+0.04}$ & $-0.62_{-0.11}^{+0.10}$ & \\
\hline J095509.6+174124 & 0112850101 & $095509.6+174124.5$ & 3.80 & $1.41 \pm 0.10$ & $-0.55_{-0.06}^{+0.06}$ & $-0.51_{-0.11}^{+0.11}$ & \\
\hline J095606.4+411814 & 0111290201 & $095606.5+411814.7$ & 9.12 & $1.23 \pm 0.15$ & $-0.57_{-0.10}^{+0.00}$ & $-0.62_{-0.17}^{+0.11}$ & \\
\hline J095955.2+251549 & 0041170201 & $095955.3+251549.1$ & 10.40 & $1.62 \pm 0.10$ & $-0.77_{-0.04}^{-0.04}$ & $-0.84_{-0.09}^{+0.09}$ & star \\
\hline $\mathrm{J} 100020.6+252046$ & 0041170201 & $100020.6+252046.7$ & 7.83 & $2.37 \pm 0.10$ & $-0.78_{-0.03}^{+0.02}$ & $-0.45_{-0.09}^{+0.09}$ & \\
\hline $\mathrm{J} 100032.5+553626$ & 0110930201 & $100032.5+553626.4$ & 12.64 & $1.90 \pm 0.27$ & $-0.48_{-0.11}^{+0.11}$ & $-0.30_{-0.21}^{+0.21}$ & AGN2 \\
\hline $\mathrm{J} 100100.0+252103$ & 0041170201 & $100100.1+252103.4$ & 7.94 & $1.37 \pm 0.07$ & $-0.65_{-0.04}^{+0.04}$ & $-0.60_{-0.09}^{+0.08}$ & \\
\hline $\mathrm{J} 100309.4+554135$ & 0110930201 & $100309.5+554135.3$ & 10.26 & $1.48 \pm 0.22$ & $-0.47_{-0.13}^{-0.04}$ & $-0.62_{-0.20}^{+0.09}$ & AGN1 \\
\hline $\mathrm{J} 100828.8+535408$ & 0070340201 & $100828.8+535408.3$ & 12.42 & $1.09 \pm 0.11$ & $-0.60_{-0.08}^{+0.08}$ & $-0.38_{-0.17}^{+0.17}$ & \\
\hline $\mathrm{J} 100921.7+534926$ & 0070340201 & $100921.8+534926.7$ & 9.03 & $1.82 \pm 0.13$ & $-0.66_{-0.05}^{+0.05}$ & $-0.59_{-0.12}^{+0.11}$ & AGN1 \\
\hline
\end{tabular}


Table 3. continued.

\begin{tabular}{|c|c|c|c|c|c|c|c|}
\hline $\begin{array}{l}\text { Name } \\
\text { XBS... } \\
(1)\end{array}$ & Obs. ID & RA; Dec (J2000) & $\begin{array}{r}\text { OffAxis } \\
\operatorname{arcmin} \\
(4)\end{array}$ & $\begin{array}{r}\text { Rate } \\
\times 10^{-2} \mathrm{cts} / \mathrm{s} \\
(5)\end{array}$ & $H R 2$ & $H R 3$ & Class \\
\hline J100926.5+533426 & 0070340201 & $100926.6+533426.1$ & 9.57 & $1.36 \pm 0.12$ & $-0.45_{-0.08}^{+0.08}$ & $-0.46_{-0.13}^{+0.12}$ & AGN1 \\
\hline $\mathrm{J} 101506.0+520157$ & 0086750101 & $101506.0+520157.9$ & 13.00 & $1.41 \pm 0.27$ & $-0.59_{-0.14}^{+0.14}$ & $-1.00_{-0.00}^{+0.63}$ & \\
\hline $\mathrm{J} 101511.8+520708^{d}$ & 0086750101 & $101511.8+520708.0$ & 10.02 & $1.72 \pm 0.27$ & $-0.65_{-0.10}^{+0.10}$ & $-0.71_{-0.26}^{+0.20}$ & AGN1 \\
\hline $\mathrm{J} 101706.5+520245^{d}$ & 0086750101 & $101706.5+520245.6$ & 10.28 & $1.83 \pm 0.24$ & $-0.66_{-0.09}^{+0.09}$ & $-0.99_{-0.01}^{+0.36}$ & $\mathrm{BL}$ \\
\hline $\mathrm{J} 101838.0+411635$ & 0028740301 & $101838.0+411635.6$ & 8.67 & $1.43 \pm 0.10$ & $-0.71_{-0.04}^{+0.04}$ & $-0.73_{-0.11}^{+0.11}$ & \\
\hline $\mathrm{J} 101843.0+413515$ & 0028740301 & $101843.1+413515.5$ & 10.02 & $1.93 \pm 0.13$ & $-0.60_{-0.05}^{+0.04}$ & $-0.52_{-0.11}^{+0.10}$ & AGN2? \\
\hline $\mathrm{J} 101850.5+411506$ & 0028740301 & $101850.6+411506.6$ & 10.32 & $4.33 \pm 0.18$ & $-0.69_{-0.03}^{+0.03}$ & $-0.51_{-0.08}^{+0.07}$ & AGN1 \\
\hline J101922.6+412049 & 0028740301 & $101922.6+412049.7$ & 9.06 & $3.36 \pm 0.14$ & $-0.44_{-0.04}^{+0.04}$ & $-0.58_{-0.05}^{+0.06}$ & AGN1 \\
\hline $\mathrm{J} 102016.1+082143^{d}$ & 0093640301 & $102016.1+082143.9$ & 9.13 & $1.14 \pm 0.14$ & $-0.40_{-0.10}^{+0.10}$ & $-0.47_{-0.15}^{+0.15}$ & \\
\hline $\mathrm{J} 102044.1+081424$ & 0093640301 & $102044.1+081424.7$ & 10.99 & $3.35 \pm 0.26$ & $-0.94_{-0.03}^{+0.03}$ & $-1.00_{-0.00}^{+0.75}$ & star \\
\hline $\mathrm{J} 102252.0+194837$ & 0101040301 & $102252.1+194837.9$ & 9.65 & $1.42 \pm 0.10$ & $-0.48_{-0.06}^{+0.06}$ & $-0.51_{-0.09}^{+0.09}$ & \\
\hline $\mathrm{J} 102412.3+042023$ & 0108670101 & $102412.3+042023.9$ & 12.07 & $1.08 \pm 0.07$ & $-0.48_{-0.06}^{+0.06}$ & $-0.68_{-0.08}^{+0.08}$ & AGN1? \\
\hline $\mathrm{J} 102417.5+041656$ & 0108670101 & $102417.5+041656.4$ & 10.88 & $1.60 \pm 0.08$ & $-0.55_{-0.04}^{+0.06}$ & $-0.44_{-0.08}^{+0.08}$ & \\
\hline $\mathrm{J} 103120.0+311404$ & 0102040301 & $103120.0+311404.1$ & 12.08 & $1.68 \pm 0.13$ & $-0.69_{-0.05}^{+0.04}$ & $-0.28_{-0.14}^{+0.14}$ & \\
\hline $\mathrm{J} 103154.1+310732$ & 0102040301 & $103154.1+310732.2$ & 12.70 & $1.03 \pm 0.10$ & $-0.34_{-0.08}^{+0.08}$ & $-0.46_{-0.12}^{+0.14}$ & AGN1 \\
\hline $\mathrm{J} 103745.7+532353$ & 0112810301 & $103745.8+532353.8$ & 10.73 & $1.03 \pm 0.18$ & $-0.71_{-0.14}^{+0.14}$ & $-0.18_{-0.43}^{+0.44}$ & \\
\hline $\mathrm{J} 103909.4+205222$ & 0059800101 & $103909.4+205222.0$ & 10.44 & $2.25 \pm 0.20$ & $-0.55_{-0.07}^{+0.07}$ & $-0.47_{-0.14}^{+0.14}$ & \\
\hline $\mathrm{J} 103932.7+205426$ & 0059800101 & $103932.8+205426.0$ & 6.04 & $1.94 \pm 0.15$ & $-0.51_{-0.06}^{+0.06}$ & $-0.67_{-0.10}^{+0.14}$ & \\
\hline $\mathrm{J} 103935.8+533036$ & 0112810301 & $103935.9+533036.9$ & 7.67 & $2.72 \pm 0.31$ & $\begin{array}{r}-0.06 \\
-0.55_{-0.09}^{+0.09}\end{array}$ & $-0.71_{-0.16}^{+0.10}$ & AGN1 \\
\hline $\mathrm{J} 103958.3+203848$ & 0059800101 & $103958.3+203848.2$ & 12.11 & $1.11 \pm 0.14$ & $-0.43_{-0.11}^{+0.10}$ & $-0.42_{-0.17}^{+0.17}$ & \\
\hline $\mathrm{J} 104026.9+204542$ & 0059800101 & $104026.9+204543.0$ & 9.32 & $11.76 \pm 0.37$ & $-0.58_{-0.03}^{+0.03}$ & $-0.48_{-0.05}^{+0.05}$ & AGN1 \\
\hline $\mathrm{J} 104034.3+205110$ & 0059800101 & $104034.4+205110.4$ & 9.51 & $1.16 \pm 0.12$ & $-0.45_{-0.09}^{+0.09}$ & $-0.76_{-0.12}^{+0.11}$ & \\
\hline J104425.0-013521 & 0125300101 & $104425.0-013521.2$ & 10.43 & $1.19 \pm 0.09$ & $-0.56_{-0.06}^{+0.06}$ & $-0.52_{-0.12}^{+0.12}$ & AGN1 \\
\hline $\mathrm{J} 104451.5-012227$ & 0125300101 & $104451.5-012227.7$ & 5.45 & $1.23 \pm 0.07$ & $-0.41_{-0.05}^{+0.05}$ & $-0.57_{-0.08}^{+0.08}$ & \\
\hline J104509.3-012442 & 0125300101 & $104509.3-012442.3$ & 9.23 & $1.02 \pm 0.08$ & $-0.73_{-0.05}^{+0.05}$ & $-0.68_{-0.14}^{+0.14}$ & \\
\hline $\mathrm{J} 104522.1-012843$ & 0125300101 & $104522.1-012843.3$ & 12.94 & $4.45 \pm 0.22$ & $-0.63_{-0.04}^{+0.04}$ & $-0.46_{-0.08}^{+0.08}$ & AGN1 \\
\hline $\mathrm{J} 104912.8+330459$ & 0055990201 & $104912.8+330459.8$ & 10.46 & $2.57 \pm 0.18$ & $-0.45_{-0.06}^{+0.06}$ & $-0.54_{-0.10}^{+0.09}$ & AGN1? \\
\hline $\mathrm{J} 105014.9+331013$ & 0055990201 & $105014.9+331013.2$ & 12.11 & $1.51 \pm 0.15$ & $-0.55_{-0.08}^{+0.08}$ & $-0.61_{-0.14}^{+0.10}$ & \\
\hline $\mathrm{J} 105131.1+573439$ & 0022740101 & $105131.1+573439.1$ & 11.41 & $2.06 \pm 0.10$ & $-0.89_{-0.02}^{+0.02}$ & $-0.33_{-0.13}^{+0.13}$ & star \\
\hline $\mathrm{J} 105239.7+572431$ & 0022740101 & $105239.8+572431.1$ & 4.33 & $1.68 \pm 0.06$ & $-0.65_{-0.02}^{+0.02}$ & $-0.62_{-0.05}^{+0.05}$ & AGN1 \\
\hline $\mathrm{J} 105316.9+573551$ & 0022740101 & $105317.0+573551.6$ & 8.31 & $2.48 \pm 0.09$ & $-0.59_{-0.02}^{+0.02}$ & $-0.44_{-0.05}^{+0.05}$ & AGN1 \\
\hline $\mathrm{J} 105335.0+572540$ & 0022740101 & $105335.1+572540.7$ & 7.53 & $1.77 \pm 0.07$ & $-0.56_{-0.03}^{+0.03}$ & $-0.54_{-0.05}^{+0.05}$ & AGN1 \\
\hline $\mathrm{J} 105339.7+573104$ & 0022740101 & $105339.7+573104.3$ & 7.80 & $1.11 \pm 0.06$ & $-0.68_{-0.03}^{+0.03}$ & $-0.26_{-0.08}^{+0.08}$ & AGN1 \\
\hline $\mathrm{J} 105624.2-033522$ & 0094800101 & $105624.3-033522.9$ & 9.05 & $1.34 \pm 0.09$ & $-0.57_{-0.05}^{+0.05}$ & $-0.73_{-0.08}^{+0.08}$ & \\
\hline J110050.6-344331 & 0112880201 & $110050.6-344332.0$ & 12.71 & $3.97 \pm 0.18$ & $-0.48_{-0.04}^{+0.04}$ & $-0.33_{-0.07}^{+0.07}$ & \\
\hline J110119.0-345303 & 0112880201 & $110119.1-345303.8$ & 12.61 & $1.33 \pm 0.13$ & $-0.73_{-0.06}^{+0.06}$ & $-0.40_{-0.18}^{+0.17}$ & \\
\hline J110244.1-344604 & 0112880201 & $110244.2-344604.7$ & 11.26 & $1.17 \pm 0.11$ & $-0.49_{-0.08}^{+0.08}$ & $-0.71_{-0.11}^{+0.18}$ & \\
\hline $\mathrm{J} 110320.1+355803$ & 0070340301 & $110320.1+355803.7$ & 4.40 & $7.76 \pm 0.32$ & $-0.98_{-0.01}^{+0.01}$ & $-1.00_{-0.00}^{+0.76}$ & star \\
\hline J110652.0-182738 & 0112630101 & $110652.0-182738.9$ & 7.47 & $1.14 \pm 0.12$ & $-0.52_{-0.09}^{+0.09}$ & $-0.99_{-0.01}^{+0.20}$ & \\
\hline $\mathrm{J} 111654.8+180304$ & 0099030101 & $111654.8+180304.6$ & 7.71 & $1.29 \pm 0.13$ & $-0.76_{-0.05}^{+0.06}$ & $-0.66_{-0.17}^{+0.16}$ & AGN2 \\
\hline $\mathrm{J} 111928.5+130250^{d}$ & 0093641101 & $111928.6+130250.7$ & 11.81 & $1.74 \pm 0.18$ & $-0.60_{-0.08}^{+0.08}$ & $-0.29_{-0.18}^{+0.19}$ & AGN1 \\
\hline $\mathrm{J} 111933.0+212756$ & 0111290401 & $111933.0+212756.7$ & 10.33 & $1.59 \pm 0.18$ & $-0.42_{-0.11}^{+0.08}$ & $-0.70_{-0.15}^{+0.18}$ & AGN1 \\
\hline $\mathrm{J} 111942.1+211516$ & 0111290401 & $111942.2+211516.2$ & 8.75 & $1.01 \pm 0.14$ & $-0.76_{-0.09}^{+0.09}$ & $0.17_{-0.27}^{+0.26}$ & AGN1 \\
\hline $\mathrm{J} 112022.3+125252$ & 0093641101 & $112022.3+125252.7$ & 6.84 & $2.56 \pm 0.19$ & $-0.66_{-0.06}^{+0.06}$ & $-0.61_{-0.13}^{+0.13}$ & AGN1 \\
\hline J112046.7+125429 & 0093641101 & $112046.8+125430.0$ & 9.21 & $3.07 \pm 0.24$ & $-0.67_{-0.06}^{+0.06}$ & $-0.66_{-0.14}^{+0.13}$ & AGN1 \\
\hline $\mathrm{J} 113106.9+312518$ & 0102040201 & $113107.0+312518.2$ & 11.18 & $1.33 \pm 0.12$ & $-0.53_{-0.08}^{+0.00}$ & $-0.15_{-0.14}^{+0.14}$ & AGN1 \\
\hline $\mathrm{J} 113121.8+310252$ & 0102040201 & $113121.8+310252.6$ & 11.58 & $1.67 \pm 0.12$ & $-0.03_{-0.07}^{+0.07}$ & $-0.35_{-0.08}^{+0.08}$ & AGN2 \\
\hline J113128.6-195903 & 0042341001 & $113128.7-195903.4$ & 7.35 & $1.50 \pm 0.13$ & $-0.67_{-0.07}^{+0.07}$ & $-0.73_{-0.14}^{+0.14}$ & \\
\hline $\mathrm{J} 113148.7+311358$ & 0102040201 & $113148.7+311358.8$ & 8.45 & $1.54 \pm 0.10$ & $0.10_{-0.07}^{+0.06}$ & $-0.43_{-0.07}^{+0.07}$ & AGN2 \\
\hline J113837.9-373402 & 0112210101 & $113837.9-373402.0$ & 11.33 & $1.79 \pm 0.12$ & $-0.60_{-0.05}^{+0.05}$ & $-0.44_{-0.10}^{+0.11}$ & AGN1? \\
\hline $\mathrm{J} 115317.9+364712$ & 0112551401 & $115317.9+364712.5$ & 12.82 & $1.08 \pm 0.21$ & $-0.46_{-0.19}^{+0.19}$ & $-1.00_{-0.00}^{+0.10}$ & \\
\hline $\mathrm{J} 115846.9+551625$ & 0090020101 & $115846.9+551625.4$ & 12.98 & $1.74 \pm 0.25$ & $-0.65_{-0.10}^{+0.10}$ & $-0.98_{-0.02}^{+0.45}$ & AGN1 \\
\hline $\mathrm{J} 120359.1+443715$ & 0109141401 & $120359.2+443715.1$ & 10.37 & $1.37 \pm 0.07$ & $-0.73_{-0.03}^{+0.03}$ & $-0.63_{-0.09}^{+0.09}$ & \\
\hline $\mathrm{J} 120413.7+443149$ & 0109141401 & $120413.8+443149.7$ & 11.47 & $1.28 \pm 0.08$ & $-0.60_{-0.04}^{+0.05}$ & $-0.70_{-0.08}^{+0.08}$ & \\
\hline
\end{tabular}


Table 3. continued.

\begin{tabular}{|c|c|c|c|c|c|c|c|}
\hline $\begin{array}{l}\text { Name } \\
\text { XBS... } \\
\text { (1) }\end{array}$ & Obs. ID & RA; Dec (J2000) & $\begin{array}{r}\text { OffAxis } \\
\operatorname{arcmin} \\
(4)\end{array}$ & $\begin{array}{r}\text { Rate } \\
\times 10^{-2} \mathrm{cts} / \mathrm{s} \\
(5)\end{array}$ & $H R 2$ & $H R 3$ & Class \\
\hline $\mathrm{J} 121501.7+140113$ & 0112610101 & $121501.8+140113.7$ & 11.06 & $1.84 \pm 0.10$ & $-0.58_{-0.04}^{+0.04}$ & $-0.68_{-0.08}^{+0.07}$ & \\
\hline $\mathrm{J} 122017.5+752217^{c}$ & 0124110101 & $122017.5+752217.8$ & 6.45 & $10.78 \pm 0.35$ & $-0.87_{-0.03}^{+0.03}$ & $-1.0_{-0.12}^{+0.0}$ & GAL \\
\hline $\mathrm{J} 122350.4+752231$ & 0124110101 & $122350.5+752231.7$ & 9.04 & $1.49 \pm 0.13$ & $-0.55_{-0.07}^{+0.07}$ & $-0.72_{-0.11}^{+0.11}$ & AGN1 \\
\hline $\mathrm{J} 122628.9+333626$ & 0070340501 & $122628.9+333626.6$ & 10.57 & $1.08 \pm 0.13$ & $-0.83_{-0.06}^{+0.07}$ & $-0.64_{-0.32}^{+0.11}$ & \\
\hline $\mathrm{J} 122655.1+012002$ & 0110990201 & $122655.1+012002.8$ & 10.96 & $1.32 \pm 0.16$ & $-0.80_{-0.07}^{+0.00}$ & $-0.78_{-0.20}^{+0.22}$ & \\
\hline $\mathrm{J} 122656.5+013126$ & 0110990201 & $122656.6+013126.2$ & 5.85 & $1.41 \pm 0.12$ & $-0.11_{-0.09}^{+0.09}$ & $-0.34_{-0.10}^{+0.10}$ & AGN2? \\
\hline $\mathrm{J} 122658.1+333246^{c, d}$ & 0070340501 & $122658.1+333246.5$ & 3.68 & $7.15 \pm 0.25$ & $-0.51_{-0.04}^{+0.04}$ & $-0.80_{-0.04}^{+0.04}$ & CL \\
\hline $\mathrm{J} 122751.2+333842$ & 0070340501 & $122751.3+333843.0$ & 9.80 & $1.87 \pm 0.17$ & $-0.85_{-0.04}^{+0.04}$ & $-0.39_{-0.23}^{+0.04}$ & star \\
\hline $\mathrm{J} 122803.7+333957$ & 0070340501 & $122803.7+333957.1$ & 12.60 & $1.15 \pm 0.16$ & $\begin{array}{l}-0.08_{-0.10}^{+0.04} \\
-0.58_{-10}^{+0.10}\end{array}$ & $\begin{array}{r}-0.23 \\
-1.00_{-0.00}^{+0.31}\end{array}$ & \\
\hline $\mathrm{J} 122837.3+015720$ & 0126700301 & $122837.3+015720.6$ & 9.33 & $2.16 \pm 0.09$ & $-0.88_{-0.02}^{+0.10}$ & $-0.99_{-0.01}^{+0.00}$ & star \\
\hline J122942.3+015525 & 0126700301 & $122942.4+015525.4$ & 11.80 & $2.06 \pm 0.11$ & $-0.84_{-0.03}^{+0.03}$ & $-0.83_{-0.09}^{+0.09}$ & star \\
\hline $\mathrm{J} 123036.2+642531$ & 0124900101 & $123036.3+642531.8$ & 12.66 & $1.09 \pm 0.09$ & $-0.70_{-0.06}^{+0.05}$ & $-0.49_{-0.16}^{+0.15}$ & \\
\hline $\mathrm{J} 123116.5+641115$ & 0124900101 & $123116.5+641115.1$ & 3.46 & $1.06 \pm 0.06$ & $-0.51_{-0.05}^{-0.06}$ & $\begin{array}{r}-0.15_{-0.09}^{+0.09} \\
-0.45_{-1}^{+0.0}\end{array}$ & \\
\hline $\mathrm{J} 123208.7+640304$ & 0124900101 & $123208.8+640304.3$ & 12.00 & $1.16 \pm 0.11$ & $-0.82_{-0.05}^{+0.05}$ & $-0.91_{-0.07}^{+0.11}$ & star \\
\hline $\mathrm{J} 123218.5+640311$ & 0124900101 & $123218.6+640311.3$ & 12.30 & $1.48 \pm 0.12$ & $-0.58_{-0.06}^{+0.06}$ & $-0.52_{-0.12}^{+0.07}$ & \\
\hline $\mathrm{J} 123538.6+621644^{d}$ & 0111550101 & $123538.6+621644.7$ & 8.98 & $2.06 \pm 0.09$ & $-0.59_{-0.03}^{+0.03}$ & $-0.59_{-0.07}^{+0.07}$ & AGN1 \\
\hline J123549.1-395026 & 0006220201 & $123549.1-395026.8$ & 5.25 & $3.23 \pm 0.10$ & $-0.94_{-0.01}^{+0.01}$ & $-1.00_{-0.00}^{+0.14}$ & star \\
\hline J123600.7-395217 & 0006220201 & $123600.7-395218.0$ & 5.62 & $17.71 \pm 0.22$ & $-0.77_{-0.01}^{+0.01}$ & $-0.77_{-0.02}^{+0.02}$ & star \\
\hline $\mathrm{J} 123759.6+621102$ & 0111550101 & $123759.7+621102.9$ & 8.51 & $1.95 \pm 0.10$ & $-0.59_{-0.04}^{+0.04}$ & $-0.55_{-0.08}^{+0.02}$ & AGN1 \\
\hline $\mathrm{J} 123800.9+621338$ & 0111550101 & $123800.9+621338.5$ & 8.47 & $1.48 \pm 0.08$ & $-0.78_{-0.03}^{+0.03}$ & $-0.36_{-0.12}^{+0.13}$ & AGN1 \\
\hline $\mathrm{J} 124214.1-112512$ & 0136950201 & $124214.1-112512.3$ & 8.24 & $1.55 \pm 0.09$ & $-0.57_{-0.05}^{+0.05}$ & $-0.33_{-0.10}^{+0.10}$ & \\
\hline $\mathrm{J} 124557.6+022659$ & 0051760101 & $124557.7+022660.0$ & 10.56 & $1.34 \pm 0.17$ & $-0.59_{-0.09}^{+0.09}$ & $-0.59_{-0.17}^{+0.10}$ & \\
\hline $\mathrm{J} 124607.6+022153$ & 0051760101 & $124607.7+022153.6$ & 6.81 & $2.41 \pm 0.17$ & $-0.62_{-0.05}^{+0.09}$ & $\begin{array}{r}-0.17 \\
-1.00_{-0.00}^{+0.10}\end{array}$ & AGN1 \\
\hline $\mathrm{J} 124641.8+022412$ & 0051760101 & $124641.8+022412.1$ & 2.78 & $3.00 \pm 0.17$ & $-0.58_{-0.05}^{+0.05}$ & $-0.48_{-0.09}^{+0.09}$ & AGN1 \\
\hline J124647.9+020955 & 0051760101 & $124648.0+020955.8$ & 12.52 & $1.03 \pm 0.19$ & $-0.39_{-0.14}^{+0.14}$ & $-0.93_{-0.06}^{+0.10}$ & \\
\hline $\mathrm{J} 124903.6-061049^{d}$ & 0060370201 & $124903.6-061049.2$ & 11.63 & $2.54 \pm 0.13$ & $-0.61_{-0.04}^{-0.04}$ & $-0.55_{-0.08}^{+0.06}$ & AGN1 \\
\hline J124914.6-060910 & 0060370201 & $124914.7-060911.0$ & 9.73 & $1.06 \pm 0.08$ & $-0.50_{-0.06}^{+0.06}$ & $-0.75_{-0.08}^{+0.08}$ & \\
\hline J124938.7-060444 & 0060370201 & $124938.7-060444.9$ & 8.20 & $35.88 \pm 0.41$ & $\begin{array}{l}-0.88_{-0.01}^{+0.06} \\
-0.01\end{array}$ & $-0.85_{-0.02}^{+0.08}$ & star \\
\hline J124949.4-060722 & 0060370201 & $124949.5-060722.0$ & 11.93 & $1.35 \pm 0.11$ & $-0.58_{-0.06}^{+0.06}$ & $-0.43_{-0.12}^{+0.12}$ & \\
\hline $\mathrm{J} 125457.2+564940$ & 0081340201 & $125457.2+564940.7$ & 10.79 & $1.72 \pm 0.13$ & $-0.46_{-0.06}^{+0.06}$ & $-0.63_{-0.10}^{+0.09}$ & AGN1 \\
\hline $\mathrm{J} 125648.4+570349$ & 0081340201 & $125648.5+570349.1$ & 12.37 & $1.19 \pm 0.12$ & $\begin{array}{r}-0.06 \\
-0.38_{-0.09}^{+0.09}\end{array}$ & $-0.72_{-0.11}^{+0.10}$ & \\
\hline $\mathrm{J} 130616.8+175923$ & 0017940101 & $130616.9+175923.7$ & 8.09 & $1.01 \pm 0.06$ & $-0.50_{-0.05}^{+0.09}$ & $-0.44_{-0.08}^{+0.11}$ & \\
\hline J130619.7-233857 & 0002940101 & $130619.7-233857.9$ & 10.84 & $2.83 \pm 0.32$ & $-0.68_{-0.08}^{+0.08}$ & $-0.56_{-0.21}^{+0.08}$ & \\
\hline J130658.1-234849 & 0002940101 & $130658.2-234849.7$ & 8.60 & $1.39 \pm 0.19$ & $-0.52_{-0.11}^{+0.11}$ & $-0.51_{-0.19}^{+0.21}$ & AGN1 \\
\hline $\mathrm{J} 132038.0+341124$ & 0093640401 & $132038.1+341124.3$ & 3.10 & $2.91 \pm 0.13$ & $-0.43_{-0.04}^{+0.04}$ & $-0.48_{-0.06}^{+0.06}$ & AGN1 \\
\hline $\mathrm{J} 132052.5+341742$ & 0093640401 & $132052.5+341742.9$ & 10.01 & $1.50 \pm 0.12$ & $\begin{array}{l}-0.0 .04 \\
-0.53_{-0.07}^{+0.07}\end{array}$ & $-0.58_{-0.012}^{+0.06}$ & AGN1 \\
\hline J132101.6+340656 & 0093640401 & $132101.6+340656.6$ & 5.61 & $3.88 \pm 0.15$ & $\begin{array}{l}-0.71_{-0.03}^{+0.03} \\
-0.03\end{array}$ & $-0.57_{-0.07}^{+0.12}$ & AGN1 \\
\hline $\mathrm{J} 132105.5+341459^{d}$ & 0093640401 & $132105.5+341459.4$ & 9.10 & $2.74 \pm 0.15$ & $-0.56_{-0.05}^{+0.05}$ & $-0.56_{-0.09}^{+0.09}$ & \\
\hline $\mathrm{J} 133023.8+241707$ & 0100240201 & $133023.8+241707.3$ & 6.22 & $1.15 \pm 0.07$ & $-0.68_{-0.05}^{+0.05}$ & $-0.41_{-0.11}^{+0.12}$ & AGN1 \\
\hline $\mathrm{J} 133026.6+241520^{d}$ & 0100240201 & $133026.7+241520.5$ & 4.91 & $2.08 \pm 0.09$ & $-0.82_{-0.03}^{+0.02}$ & $-0.57_{-0.10}^{+0.10}$ & \\
\hline $\mathrm{J} 133120.2+242305$ & 0100240201 & $133120.2+242305.1$ & 11.78 & $1.77 \pm 0.11$ & $-0.56_{-0.05}^{+0.05}$ & $-0.60_{-0.09}^{+0.09}$ & \\
\hline $\mathrm{J} 133232.6+111220^{d}$ & 0061940101 & $133232.7+111220.7$ & 10.70 & $1.05 \pm 0.18$ & $-0.35_{-0.15}^{+0.15}$ & $-1.00_{-0.00}^{+0.39}$ & \\
\hline $\mathrm{J} 133321.2+503102$ & 0111160101 & $133321.3+503102.2$ & 9.21 & $1.79 \pm 0.08$ & $-0.91_{-0.02}^{+0.02}$ & $-0.49_{-0.16}^{+0.16}$ & star \\
\hline J133626.9-342636 & 0111570201 & $133626.9-342636.7$ & 11.19 & $1.00 \pm 0.08$ & $-0.90_{-0.03}^{+0.02}$ & $-1.00_{-0.00}^{+0.16}$ & star \\
\hline $\mathrm{J} 133807.5+242411$ & 0096010101 & $133807.6+242411.8$ & 11.17 & $1.69 \pm 0.11$ & $-0.66_{-0.05}^{+0.05}$ & $-0.65_{-0.10}^{+0.10}$ & AGN1 \\
\hline J133942.6-315004 & 0035940301 & $133942.6-315004.8$ & 11.91 & $1.67 \pm 0.11$ & $-0.36_{-0.06}^{+0.05}$ & $-0.20_{-0.08}^{+0.08}$ & AGN1? \\
\hline $\mathrm{J} 134732.0+582103$ & 0112250201 & $134732.0+582103.8$ & 8.38 & $3.43 \pm 0.13$ & $-0.86_{-0.02}^{+0.02}$ & $-0.81_{-008}^{+0.07}$ & star \\
\hline $\mathrm{J} 134749.9+582111$ & 0112250201 & $134749.9+582111.0$ & 8.53 & $12.44 \pm 0.24$ & $-0.60_{-0.02}^{+0.02}$ & $-0.54_{-0.03}^{+0.03}$ & AGN1 \\
\hline $\mathrm{J} 140100.0-110942$ & 0109910101 & $140100.0-110942.2$ & 8.70 & $1.12 \pm 0.07$ & $-0.57_{-0.05}^{-0.02}$ & $\begin{array}{r}-0.03 \\
-0.66_{-0.09}^{+0.09}\end{array}$ & \\
\hline J140102.0-111224 & 0109910101 & $140102.0-111224.3$ & 9.27 & $8.34 \pm 0.18$ & $-0.52_{-0.02}^{+0.02}$ & $-0.46_{-0.03}^{+0.03}$ & AGN2? \\
\hline $\mathrm{J} 140127.7+025605$ & 0098010101 & $140127.7+025605.4$ & 7.25 & $7.04 \pm 0.19$ & $-0.38_{-0.02}^{+0.02}$ & $-0.53_{-0.04}^{+0.04}$ & AGN1 \\
\hline
\end{tabular}


Table 3. continued.

\begin{tabular}{|c|c|c|c|c|c|c|c|}
\hline $\begin{array}{l}\text { Name } \\
\text { XBS... } \\
\text { (1) }\end{array}$ & Obs. ID & RA; Dec (J2000) & $\begin{array}{r}\text { OffAxis } \\
\text { arcmin } \\
\text { (4) }\end{array}$ & $\begin{array}{r}\text { Rate } \\
\times 10^{-2} \mathrm{cts} / \mathrm{s} \\
(5)\end{array}$ & $\overline{H R 2}$ & $H R 3$ & Class \\
\hline J140219.6-110458 & 0109910101 & $140219.7-110458.8$ & 11.40 & $1.16 \pm 0.08$ & $-0.94_{-0.02}^{+0.02}$ & $-0.16_{-0.27}^{+0.29}$ & star \\
\hline $\mathrm{J} 140921.1+261336$ & 0092850501 & $140921.2+261336.8$ & 4.78 & $1.24 \pm 0.06$ & $-0.32_{-0.05}^{+0.04}$ & $-0.53_{-0.06}^{+0.06}$ & \\
\hline $\mathrm{J} 140936.9+261632$ & 0092850501 & $140936.9+261632.1$ & 3.44 & $1.11 \pm 0.06$ & $-0.92_{-0.02}^{+0.02}$ & $-0.70_{-0.17}^{+0.16}$ & star \\
\hline J141235.8-030909 & 0013140101 & $141235.8-030909.3$ & 10.29 & $1.72 \pm 0.15$ & $-0.40_{-0.08}^{+0.08}$ & $-0.59_{-0.11}^{+0.11}$ & \\
\hline $\mathrm{J} 141523.8+113737$ & 0112250301 & $141523.8+113737.3$ & 9.64 & $1.16 \pm 0.09$ & $-0.70_{-0.05}^{+0.08}$ & $-0.54_{-0.15}^{+0.11}$ & \\
\hline $\mathrm{J} 141531.5+113156$ & 0112250301 & $141531.6+113156.2$ & 4.15 & $3.15 \pm 0.12$ & $-0.55_{-0.03}^{+0.03}$ & $-0.47_{-0.06}^{+0.06}$ & AGN1 \\
\hline $\mathrm{J} 141643.8+521434$ & 0127921001 & $141643.8+521434.2$ & 10.17 & $1.41 \pm 0.07$ & $-0.58_{-0.04}^{+0.04}$ & $-0.50_{-0.08}^{+0.08}$ & \\
\hline $\mathrm{J} 141658.8+521202$ & 0127921001 & $141658.8+521202.8$ & 11.98 & $1.01 \pm 0.08$ & $-0.71_{-0.05}^{+0.05}$ & $-0.28_{-0.14}^{+0.14}$ & \\
\hline $\mathrm{J} 141722.6+251335$ & 0109960101 & $141722.7+251335.2$ & 9.86 & $1.33 \pm 0.12$ & $-0.69_{-0.06}^{+0.06}$ & $-1.00_{-0.00}^{+0.14}$ & AGN1 \\
\hline J141736.3+523028 & 0127921001 & $141736.3+523028.5$ & 7.67 & $1.15 \pm 0.06$ & $-0.65_{-0.04}^{+0.04}$ & $-0.40_{-0.09}^{+0.09}$ & AGN1 \\
\hline $\mathrm{J} 141809.1+250040$ & 0109960101 & $141809.1+250040.3$ & 7.85 & $1.31 \pm 0.09$ & $-0.59_{-0.05}^{+0.06}$ & $-0.47_{-0.12}^{+0.12}$ & AGN1 \\
\hline $\mathrm{J} 141830.5+251052^{c}$ & 0109960101 & $141830.5+251052.6$ & 7.58 & $14.99 \pm 0.37$ & $-0.52_{-0.04}^{+0.03}$ & $-0.65_{-0.07}^{+0.07}$ & CL \\
\hline $\mathrm{J} 142800.1+424409$ & 0111850201 & $142800.1+424409.5$ & 7.10 & $1.53 \pm 0.07$ & $-0.90_{-0.02}^{+0.02}$ & $-0.48_{-0.16}^{+0.17}$ & star \\
\hline $\mathrm{J} 142901.2+423048$ & 0111850201 & $142901.2+423048.8$ & 10.84 & $1.70 \pm 0.11$ & $-0.98_{-0.01}^{+0.02}$ & $0.56_{-0.30}^{+0.16}$ & star \\
\hline J143835.1+642928 & 0111530101 & $143835.1+642928.3$ & 12.34 & $1.31 \pm 0.09$ & $0.18_{-0.07}^{+0.07}$ & $-0.37_{-0.06}^{+0.07}$ & AGN1? \\
\hline $\mathrm{J} 143923.1+640912$ & 0111530101 & $143923.2+640912.4$ & 8.60 & $2.36 \pm 0.09$ & $-0.99_{-0.01}^{+0.01}$ & $-0.51_{-0.46}^{+0.40}$ & star \\
\hline $\mathrm{J} 144021.0+642144$ & 0111530101 & $144021.0+642144.1$ & 9.79 & $1.42 \pm 0.07$ & $-0.47_{-0.04}^{+0.01}$ & $-0.37_{-0.08}^{+0.06}$ & \\
\hline $\mathrm{J} 144937.5+090826$ & 0057560301 & $144937.6+090826.0$ & 8.83 & $1.86 \pm 0.10$ & $-0.52_{-0.04}^{+0.04}$ & $-0.54_{-0.07}^{+0.07}$ & \\
\hline J144945.8+085921 & 0057560301 & $144945.9+085921.8$ & 4.14 & $1.04 \pm 0.06$ & $-0.56_{-0.04}^{+0.05}$ & $-0.51_{-0.09}^{+0.09}$ & \\
\hline J145857.1-313535 & 0067750101 & $145857.1-313536.0$ & 8.89 & $1.03 \pm 0.08$ & $-0.55_{-0.06}^{+0.06}$ & $-0.45_{-0.12}^{+0.12}$ & \\
\hline $\mathrm{J} 150428.3+101856$ & 0070740301 & $150428.4+101857.0$ & 9.91 & $1.67 \pm 0.17$ & $\begin{array}{r}-0.37_{-0.09}^{+0.09} \\
-0.09\end{array}$ & $-1.00_{-0.00}^{+0.12}$ & AGN1 \\
\hline $\mathrm{J} 151815.0+060851$ & 0018741001 & $151815.1+060851.5$ & 9.43 & $1.12 \pm 0.19$ & $-0.77_{-0.10}^{+0.10}$ & $0.22_{-0.32}^{+0.00}$ & \\
\hline $\mathrm{J} 151832.3+062357$ & 0018741001 & $151832.4+062357.6$ & 10.39 & $1.72 \pm 0.27$ & $-0.72_{-0.09}^{+0.10}$ & $-0.70_{-0.27}^{+0.32}$ & GAL \\
\hline J153156.6-082610 & 0100240801 & $153156.6-082611.0$ & 8.75 & $1.32 \pm 0.11$ & $-0.96_{-0.02}^{+0.02}$ & $-1.00_{-0.00}^{+0.96}$ & star \\
\hline J153205.7-082952 & 0100240801 & $153205.8-082952.6$ & 4.77 & $1.05 \pm 0.08$ & $-0.45_{-0.06}^{+0.07}$ & $-0.79_{-0.08}^{+0.08}$ & AGN1 \\
\hline $\mathrm{J} 153419.0+011808$ & 0112190401 & $153419.1+011808.2$ & 10.91 & $1.37 \pm 0.15$ & $-0.62_{-0.08}^{+0.08}$ & $-0.30_{-0.18}^{+0.18}$ & AGN1 \\
\hline $\mathrm{J} 153452.3+013104^{d}$ & 0112190401 & $153452.4+013104.6$ & 10.57 & $9.44 \pm 0.38$ & $-0.39_{-0.04}^{+0.04}$ & $-0.55_{-0.05}^{+0.05}$ & AGN1 \\
\hline $\mathrm{J} 153456.1+013033$ & 0112190401 & $153456.2+013033.4$ & 9.89 & $3.05 \pm 0.23$ & $-0.64_{-0.05}^{+0.05}$ & $-0.64_{-0.11}^{+0.11}$ & AGN1 \\
\hline $\mathrm{J} 160645.9+081525$ & 0067340601 & $160646.0+081525.1$ & 12.61 & $1.28 \pm 0.17$ & $0.81_{-0.08}^{+0.08}$ & $-0.23_{-0.10}^{+0.10}$ & AGN2? \\
\hline $\mathrm{J} 160706.6+075709$ & 0067340601 & $160706.7+075709.5$ & 7.90 & $1.49 \pm 0.14$ & $-0.65_{-0.06}^{+0.08}$ & $-0.34_{-0.16}^{+0.10}$ & AGN1 \\
\hline $\mathrm{J} 160731.5+081202$ & 0067340601 & $160731.6+081202.9$ & 8.43 & $1.67 \pm 0.14$ & $-0.75_{-0.05}^{+0.05}$ & $-0.44_{-0.18}^{+0.16}$ & AGN1? \\
\hline $\mathrm{J} 161544.2+121708$ & 0103460801 & $161544.2+121708.3$ & 11.87 & $1.41 \pm 0.18$ & $-0.64_{-0.09}^{+0.09}$ & $-0.18_{-0.24}^{+0.28}$ & \\
\hline $\mathrm{J} 161615.1+121353$ & 0103460801 & $161615.1+121353.2$ & 3.65 & $1.11 \pm 0.10$ & $-0.65_{-0.07}^{+0.07}$ & $-1.00_{-0.00}^{+0.19}$ & \\
\hline $\mathrm{J} 161825.4+124145$ & 0103461001 & $161825.5+124145.5$ & 12.82 & $1.14 \pm 0.15$ & $-0.76_{-0.09}^{+0.08}$ & $-0.17_{-0.31}^{+0.00}$ & AGN1 \\
\hline $\mathrm{J} 162813.9+780342$ & 0061940301 & $162814.0+780342.7$ & 10.92 & $2.64 \pm 0.34$ & $-0.67_{-0.09}^{+0.09}$ & $-0.33_{-0.24}^{+0.24}$ & AGN1 \\
\hline $\mathrm{J} 162911.1+780442$ & 0061940301 & $162911.2+780442.7$ & 8.13 & $1.66 \pm 0.22$ & $-0.81_{-0.08}^{+0.08}$ & $-1.00_{-0.00}^{+0.76}$ & star \\
\hline J162923.3+781306 & 0061940301 & $162923.4+781306.6$ & 5.11 & $1.41 \pm 0.18$ & $-0.19_{-0.13}^{+0.13}$ & $-0.68_{-0.14}^{+0.14}$ & \\
\hline $\mathrm{J} 162944.8+781128$ & 0061940301 & $162944.8+781128.5$ & 3.57 & $1.77 \pm 0.20$ & $-0.99_{-0.01}^{+0.02}$ & $-1.00_{-0.00}^{+2.00}$ & star \\
\hline $\mathrm{J} 163141.1+781239$ & 0061940301 & $163141.2+781239.3$ & 2.99 & $1.39 \pm 0.17$ & $-0.61_{-0.11}^{+0.10}$ & $-0.84_{-0.16}^{+0.15}$ & AGN1 \\
\hline $\mathrm{J} 163223.6+052547$ & 0112230301 & $163223.6+052547.1$ & 10.44 & $1.89 \pm 0.14$ & $-0.71_{-0.05}^{+0.05}$ & $-0.23_{-0.15}^{+0.16}$ & AGN1 \\
\hline $\mathrm{J} 163309.8+571039$ & 0049540101 & $163309.8+571039.3$ & 9.67 & $2.87 \pm 0.27$ & $-0.43_{-0.07}^{+0.05}$ & $-0.49_{-0.11}^{+0.13}$ & AGN1 \\
\hline $\mathrm{J} 163332.3+570520$ & 0049540101 & $163332.4+570520.2$ & 7.90 & $1.03 \pm 0.16$ & $-0.84_{-0.06}^{+0.06}$ & $-1.00_{-0.00}^{+0.63}$ & AGN1? \\
\hline $\mathrm{J} 163427.5+781002$ & 0061940301 & $163427.6+781002.5$ & 11.00 & $1.79 \pm 0.30$ & $-0.62_{-0.14}^{+0.13}$ & $-0.71_{-0.26}^{+0.24}$ & AGN1 \\
\hline $\mathrm{J} 164237.9+030014^{d}$ & 0067340501 & $164238.0+030014.3$ & 11.90 & $1.22 \pm 0.14$ & $-0.63_{-0.08}^{+0.09}$ & $-0.38_{-0.20}^{+0.26}$ & \\
\hline $\mathrm{J} 165313.3+021645$ & 0101640101 & $165313.3+021645.6$ & 8.16 & $1.36 \pm 0.13$ & $-0.96_{-0.02}^{+0.03}$ & $-1.00_{-0.00}^{+1.30}$ & star \\
\hline $\mathrm{J} 165314.4+141943$ & 0113070101 & $165314.5+141943.6$ & 11.13 & $1.54 \pm 0.21$ & $-0.42_{-0.12}^{+0.02}$ & $-0.67_{-0.16}^{+0.17}$ & \\
\hline J165406.6+142123 & 0113070101 & $165406.6+142123.6$ & 3.89 & $1.12 \pm 0.13$ & $-0.49_{-0.10}^{+0.11}$ & $-0.76_{-0.14}^{+0.14}$ & \\
\hline $\mathrm{J} 165425.3+142159$ & 0113070101 & $165425.4+142159.3$ & 7.44 & $11.86 \pm 0.53$ & $-0.63_{-0.03}^{+0.03}$ & $-0.61_{-0.07}^{+0.07}$ & AGN1 \\
\hline $\mathrm{J} 165448.5+141311$ & 0113070101 & $165448.5+141311.6$ & 12.71 & $6.78 \pm 0.56$ & $-0.54_{-0.07}^{-0.03}$ & $-0.42_{-0.13}^{+0.07}$ & AGN2 \\
\hline $\mathrm{J} 165710.5+352024$ & 0111060101 & $165710.5+352024.0$ & 7.98 & $1.93 \pm 0.20$ & $-0.90_{-0.04}^{+0.04}$ & $-1.00_{-0.00}^{+0.13}$ & star \\
\hline $\mathrm{J} 165800.7+352333$ & 0111060101 & $165800.7+352333.5$ & 3.73 & $1.40 \pm 0.13$ & $-0.51_{-0.08}^{+0.08}$ & $-0.69_{-0.13}^{+0.12}$ & AGN2? \\
\hline $\mathrm{J} 172230.6+341344$ & 0102040101 & $172230.7+341344.0$ & 11.20 & $1.74 \pm 0.25$ & $-0.65_{-0.11}^{+0.10}$ & $-0.31_{-0.27}^{+0.28}$ & AGN1 \\
\hline $\mathrm{J} 185518.7-462504$ & 0067340101 & $185518.8-462504.0$ & 6.69 & $1.55 \pm 0.20$ & $-0.40_{-0.09}^{+0.09}$ & $-1.00_{-0.00}^{+0.13}$ & AGN1 \\
\hline $\mathrm{J} 185613.7-462239^{a}$ & 0067340101 & $185613.8-462239.2$ & 10.44 & $3.02 \pm 0.30$ & $-0.47_{-0.07}^{+0.07}$ & $0.01_{-0.10}^{+0.11}$ & AGN1 \\
\hline J193138.9-725115 & 0081341001 & $193139.0-725115.0$ & 12.10 & $1.48 \pm 0.16$ & $-0.42_{-0.09}^{+0.09}$ & $-0.62_{-0.14}^{+0.10}$ & AGN1 \\
\hline
\end{tabular}


Table 3. continued.

\begin{tabular}{|c|c|c|c|c|c|c|c|}
\hline $\begin{array}{l}\text { Name } \\
\text { XBS... } \\
\text { (1) }\end{array}$ & Obs. ID & RA; Dec (J2000) & $\begin{array}{r}\text { OffAxis } \\
\operatorname{arcmin} \\
(4)\end{array}$ & $\begin{array}{r}\text { Rate } \\
\times 10^{-2} \mathrm{cts} / \mathrm{s} \\
(5)\end{array}$ & $H R 2$ & HR3 & Class \\
\hline $\mathrm{J} 193248.8-723355^{a}$ & 0081341001 & $193248.8-723355.2$ & 8.39 & $1.37 \pm 0.12$ & $-0.23_{-0.08}^{+0.08}$ & $-0.08_{-0.10}^{+0.10}$ & AGN2? \\
\hline $\mathrm{J} 204043.4-004548^{a}$ & 0111180201 & $204043.5-004548.2$ & 10.55 & $1.29 \pm 0.15$ & $0.04_{-0.11}^{+0.11}$ & $-0.35_{-0.12}^{+0.12}$ & AGN2 \\
\hline J204047.5-005853 & 0111180201 & $204047.6-005853.8$ & 11.50 & $1.05 \pm 0.14$ & $-0.33_{-0.12}^{+0.12}$ & $-0.64_{-0.15}^{+0.14}$ & BL? \\
\hline J204159.2-321439 & 0111510101 & $204159.3-321440.0$ & 11.80 & $3.36 \pm 0.23$ & $-0.54_{-0.05}^{+0.12}$ & $-0.79_{-0.07}^{+0.08}$ & AGN1 \\
\hline J204204.1-321601 & 0111510101 & $204204.1-321601.5$ & 10.68 & $1.39 \pm 0.14$ & $-0.52_{-0.08}^{+0.08}$ & $-0.80_{-0.11}^{+0.10}$ & AGN1 \\
\hline J204208.2-323523 & 0111510101 & $204208.3-323523.6$ & 9.78 & $1.16 \pm 0.12$ & $-0.63_{-0.08}^{+0.08}$ & $-0.89_{-0.09}^{+0.10}$ & AGN1 \\
\hline J204548.4-025234 & 0112600501 & $204548.4-025234.4$ & 8.74 & $1.29 \pm 0.14$ & $-0.38_{-0.10}^{+0.10}$ & $-0.76_{-0.11}^{+0.12}$ & AGN1 \\
\hline J205411.9-160804 & 0083210101 & $205411.9-160804.8$ & 12.55 & $1.97 \pm 0.23$ & $-0.58_{-0.09}^{+0.09}$ & $-0.69_{-0.16}^{+0.16}$ & AGN1 \\
\hline J205429.9-154937 & 0083210101 & $205430.0-154937.8$ & 6.59 & $1.14 \pm 0.13$ & $-0.65_{-0.08}^{+0.08}$ & $-0.80_{-0.19}^{+0.10}$ & AGN1 \\
\hline $\mathrm{J} 205635.7-044717^{a}$ & 0112190601 & $205635.8-044717.9$ & 9.96 & $3.41 \pm 0.21$ & $-0.65_{-0.05}^{+0.04}$ & $-0.48_{-0.11}^{+0.11}$ & AGN1 \\
\hline $\mathrm{J} 205829.9-423634^{a}$ & 0081340401 & $205830.0-423635.0$ & 2.42 & $5.01 \pm 0.19$ & $-0.57_{-0.03}^{+0.03}$ & $-0.47_{-0.06}^{+0.06}$ & AGN1 \\
\hline J205847.0-423704 & 0081340401 & $205847.0-423704.7$ & 4.11 & $1.71 \pm 0.12$ & $-0.93_{-0.03}^{+0.03}$ & $-1.00_{-0.00}^{+0.54}$ & star \\
\hline $\mathrm{J} 210325.4-112011$ & 0041150101 & $210325.4-112011.5$ & 11.29 & $1.01 \pm 0.09$ & $-0.49_{-0.07}^{+0.03}$ & $-0.22_{-0.13}^{+0.12}$ & AGN1 \\
\hline $\mathrm{J} 210355.3-121858$ & 0038540301 & $210355.3-121858.2$ & 11.02 & $1.56 \pm 0.17$ & $-0.50_{-0.09}^{+0.09}$ & $-0.80_{-0.10}^{+0.12}$ & AGN1 \\
\hline $\mathrm{J} 212635.8-445046$ & 0088020201 & $212635.8-445046.1$ & 11.25 & $3.03 \pm 0.25$ & $-0.92_{-0.03}^{+0.03}$ & $-0.37_{-0.32}^{+0.32}$ & star \\
\hline J212759.5-443924 & 0088020201 & $212759.6-443924.7$ & 9.99 & $1.08 \pm 0.12$ & $-0.65_{-0.08}^{+0.08}$ & $-0.47_{-0.19}^{+0.18}$ & \\
\hline $\mathrm{J} 213002.3-153414^{a}$ & 0103060101 & $213002.3-153414.1$ & 12.83 & $3.95 \pm 0.21$ & $-0.65_{-0.04}^{+0.04}$ & $-0.50_{-0.09}^{+0.09}$ & AGN1 \\
\hline J213719.6-433347 & 0109463501 & $213719.6-433347.0$ & 10.85 & $2.41 \pm 0.27$ & $-0.27_{-0.10}^{+0.10}$ & $-0.74_{-0.11}^{+0.11}$ & AGN1 \\
\hline J213729.7-423601 & 0061940201 & $213729.8-423601.6$ & 7.02 & $1.51 \pm 0.23$ & $-0.56_{-0.13}^{+0.12}$ & $-0.33_{-0.27}^{+0.15}$ & \\
\hline J213733.2-434800 & 0109463501 & $213733.2-434800.9$ & 7.07 & $1.03 \pm 0.14$ & $-0.91_{-0.06}^{+0.06}$ & $0.05_{-0.52}^{+0.48}$ & AGN1 \\
\hline J213757.6-422334 & 0061940201 & $213757.6-422334.2$ & 12.68 & $1.37 \pm 0.27$ & $-0.59_{-0.16}^{+0.15}$ & $-0.15_{-0.34}^{+0.36}$ & \\
\hline $\mathrm{J} 213820.2-142536$ & 0092850201 & $213820.2-142537.0$ & 11.17 & $2.10 \pm 0.17$ & $-0.29_{-0.07}^{+0.07}$ & $-0.45_{-0.10}^{+0.34}$ & AGN1 \\
\hline $\mathrm{J} 213824.0-423019^{a}$ & 0061940201 & $213824.0-423019.2$ & 6.50 & $2.88 \pm 0.27$ & $-0.62_{-0.08}^{+0.08}$ & $-0.36_{-0.18}^{+0.18}$ & AGN1 \\
\hline J213829.8-423958 & 0061940201 & $213829.9-423958.9$ & 5.59 & $1.45 \pm 0.19$ & $-0.66_{-0.10}^{+0.10}$ & $-0.50_{-0.24}^{+0.18}$ & AGN1 \\
\hline $\mathrm{J} 213840.5-424241$ & 0061940201 & $213840.6-424241.3$ & 8.91 & $1.89 \pm 0.26$ & $-1.00_{-0.00}^{+0.07}$ & - & star \\
\hline $\mathrm{J} 213852.2-434714$ & 0109463501 & $213852.3-434714.9$ & 11.21 & $1.32 \pm 0.23$ & $-0.68_{-0.12}^{+0.11}$ & $-0.36_{-0.33}^{+0.31}$ & AGN1 \\
\hline $\mathrm{J} 214041.4-234720^{a}$ & 0008830101 & $214041.5-234720.1$ & 9.80 & $3.46 \pm 0.22$ & $-0.57_{-0.05}^{+0.12}$ & $-0.39_{-0.10}^{+0.10}$ & AGN1 \\
\hline J215218.0-302721 & 0103060401 & $215218.1-302721.6$ & 4.80 & $1.15 \pm 0.08$ & $-0.69_{-0.05}^{+0.05}$ & $-0.55_{-0.12}^{+0.12}$ & \\
\hline $\mathrm{J} 215244.2-302407$ & 0103060401 & $215244.3-302407.4$ & 11.03 & $3.28 \pm 0.16$ & $-0.65_{-0.04}^{+0.04}$ & $-0.68_{-0.07}^{+0.08}$ & AGN1 \\
\hline $\mathrm{J} 215323.7+173018$ & 0111270101 & $215323.7+173018.6$ & 12.09 & $1.08 \pm 0.16$ & $-0.37_{-0.13}^{+0.12}$ & $-0.73_{-0.14}^{+0.16}$ & star \\
\hline $\mathrm{J} 220320.8+184930$ & 0130920101 & $220320.9+184930.3$ & 4.03 & $1.58 \pm 0.11$ & $-0.49_{-0.06}^{+0.06}$ & $-0.39_{-0.10}^{+0.14}$ & AGN2? \\
\hline J220446.8-014535 & 0012440301 & $220446.8-014535.6$ & 11.22 & $1.23 \pm 0.10$ & $-0.45_{-0.07}^{+0.06}$ & $-0.69_{-0.09}^{+0.10}$ & AGN1 \\
\hline $\mathrm{J} 220601.5-015346^{a}$ & 0012440301 & $220601.5-015347.0$ & 12.92 & $1.85 \pm 0.12$ & $-0.41_{-0.06}^{+0.06}$ & $-0.41_{-0.09}^{+0.09}$ & AGN1 \\
\hline J221623.3-174317 & 0106660101 & $221623.4-174317.9$ & 12.29 & $1.15 \pm 0.08$ & $-0.55_{-0.06}^{+0.05}$ & $-0.57_{-0.10}^{+0.10}$ & AGN1 \\
\hline J221722.4-082018 & 0009650201 & $221722.5-082018.2$ & 8.18 & $1.76 \pm 0.13$ & $-0.38_{-0.07}^{+0.00}$ & $-0.48_{-0.10}^{+0.10}$ & AGN1 \\
\hline $\mathrm{J} 221729.3-081154$ & 0009650201 & $221729.4-081154.2$ & 11.12 & $2.00 \pm 0.16$ & $-0.54_{-0.06}^{+0.06}$ & $-0.53_{-0.11}^{+0.10}$ & AGN1 \\
\hline $\mathrm{J} 221750.4-083210$ & 0009650201 & $221750.4-083210.8$ & 11.28 & $1.32 \pm 0.12$ & $-0.79_{-0.06}^{+0.06}$ & $-1.00_{-0.00}^{+0.37}$ & star \\
\hline J221821.9-081332 & 0009650201 & $221822.0-081333.0$ & 9.91 & $2.10 \pm 0.13$ & $-0.63_{-0.05}^{+0.05}$ & $-0.85_{-0.07}^{+0.07}$ & AGN1 \\
\hline $\mathrm{J} 221951.6+120123^{d}$ & 0103861201 & $221951.6+120123.1$ & 10.33 & $1.33 \pm 0.15$ & $-0.36_{-0.10}^{+0.10}$ & $-0.39_{-0.15}^{+0.15}$ & AGN2 \\
\hline J222852.2-050915 & 0100440101 & $222852.3-050915.4$ & 11.01 & $1.33 \pm 0.08$ & $-0.97_{-0.01}^{+0.01}$ & $-1.00_{-0.00}^{+0.15}$ & star \\
\hline J223547.9-255836 & 0111790101 & $223548.0-255837.0$ & 4.41 & $1.21 \pm 0.06$ & $-0.55_{-0.04}^{+0.01}$ & $\begin{array}{r}-0.00 \\
-0.50_{-0.08}^{+0.08}\end{array}$ & AGN1 \\
\hline $\mathrm{J} 223555.0-255833$ & 0111790101 & $223555.0-255833.9$ & 4.89 & $1.03 \pm 0.06$ & $-0.61_{-0.04}^{+0.04}$ & $-0.66_{-0.09}^{+0.08}$ & AGN1 \\
\hline $\mathrm{J} 223949.8+080926$ & 0103860301 & $223949.9+080926.4$ & 9.24 & $1.07 \pm 0.21$ & $-0.65_{-0.14}^{+0.14}$ & $0.09_{-0.31}^{+0.29}$ & AGN1 \\
\hline J224756.6-642721 & 0112240101 & $224756.6-642721.8$ & 12.76 & $1.43 \pm 0.11$ & $-0.51_{-0.06}^{+0.06}$ & $-0.62_{-0.10}^{+0.10}$ & \\
\hline $\mathrm{J} 224833.3-511900$ & 0109070401 & $224833.4-511900.2$ & 9.13 & $1.18 \pm 0.12$ & $-0.91_{-0.04}^{+0.06}$ & $-0.93_{-0.06}^{+0.10}$ & star \\
\hline J224846.6-505929 & 0109070401 & $224846.7-505929.6$ & 10.50 & $1.03 \pm 0.14$ & $-0.91_{-0.05}^{+0.05}$ & $-1.00_{-0.00}^{+1.30}$ & star \\
\hline $\mathrm{J} 225025.1-643225$ & 0112240101 & $225025.2-643225.3$ & 10.05 & $1.00 \pm 0.09$ & $-0.66_{-0.06}^{+0.06}$ & $-0.52_{-0.14}^{+0.14}$ & AGN1 \\
\hline $\mathrm{J} 225050.2-642900$ & 0112240101 & $225050.3-642900.4$ & 8.87 & $2.42 \pm 0.13$ & $-0.57_{-0.04}^{+0.04}$ & $-0.45_{-0.08}^{+0.08}$ & AGN1 \\
\hline $\mathrm{J} 225118.0-175951$ & 0081340901 & $225118.1-175951.3$ & 10.63 & $3.39 \pm 0.20$ & $-0.68_{-0.04}^{+0.04}$ & $-0.56_{-0.10}^{+0.10}$ & AGN1? \\
\hline $\mathrm{J} 225349.6-172137$ & 0112910301 & $225349.6-172137.2$ & 12.50 & $1.83 \pm 0.28$ & $-0.95_{-0.05}^{+0.04}$ & $\begin{array}{r}-0.10 \\
-1.00_{-0.00}^{+2.00}\end{array}$ & star \\
\hline J230400.4-083755 & 0109130701 & $230400.4-083755.5$ & 11.19 & $1.05 \pm 0.14$ & $-0.96_{-0.04}^{+0.04}$ & $-0.33_{-0.61}^{+0.00}$ & AGN1 \\
\hline $\mathrm{J} 230401.0+031519$ & 0033541001 & $230401.0+031519.2$ & 11.59 & $1.06 \pm 0.14$ & $-0.69_{-0.09}^{+0.09}$ & $-0.44_{-0.25}^{+0.01}$ & GAL \\
\hline $\mathrm{J} 230408.2+031820$ & 0033541001 & $230408.3+031820.7$ & 11.38 & $5.66 \pm 0.31$ & $-0.92_{-0.02}^{+0.02}$ & $-1.00_{-0.00}^{+0.31}$ & star \\
\hline
\end{tabular}


Table 3. continued.

\begin{tabular}{|c|c|c|c|c|c|c|c|}
\hline $\begin{array}{l}\text { Name } \\
\text { XBS... } \\
(1)\end{array}$ & Obs. ID & RA; Dec (J2000) & $\begin{array}{r}\text { OffAxis } \\
\text { arcmin } \\
(4)\end{array}$ & $\begin{array}{r}\text { Rate } \\
\times 10^{-2} \mathrm{cts} / \mathrm{s} \\
(5)\end{array}$ & $H R 2$ & HR3 & Class \\
\hline $\mathrm{J} 230434.1+122728$ & 0025541001 & $230434.1+122728.3$ & 9.68 & $1.39 \pm 0.17$ & $-0.57_{-0.10}^{+0.10}$ & $-0.31_{-0.21}^{+0.21}$ & AGN2? \\
\hline $\mathrm{J} 230443.8+121636$ & 0025541001 & $230443.9+121636.8$ & 4.30 & $1.22 \pm 0.13$ & $-0.54_{-0.09}^{+0.10}$ & $-0.77_{-0.14}^{+0.12}$ & AGN1 \\
\hline J230459.6+121205 & 0025541001 & $230459.7+121205.4$ & 7.48 & $1.35 \pm 0.15$ & $-0.49_{-0.09}^{+0.10}$ & $-0.47_{-0.16}^{+0.16}$ & AGN2? \\
\hline $\mathrm{J} 230522.1+122121$ & 0025541001 & $230522.1+122121.1$ & 6.45 & $1.28 \pm 0.14$ & $-0.37_{-0.09}^{+0.09}$ & $-0.60_{-0.13}^{+0.10}$ & AGN2 \\
\hline $\mathrm{J} 230523.0+121325$ & 0025541001 & $230523.0+121325.4$ & 8.85 & $1.31 \pm 0.15$ & $-0.47_{-0.10}^{+0.10}$ & $-0.89_{-0.11}^{+0.10}$ & \\
\hline $\mathrm{J} 231342.5-423210$ & 0123900101 & $231342.5-423210.7$ & 11.69 & $2.65 \pm 0.13$ & $-0.64_{-0.04}^{-0.04}$ & $-0.53_{-0.08}^{+0.08}$ & AGN1 \\
\hline $\mathrm{J} 231541.2-424125$ & 0093640701 & $231541.3-424125.6$ & 8.42 & $1.19 \pm 0.14$ & $-0.86_{-0.05}^{+0.05}$ & $-0.40_{-0.31}^{+0.08}$ & star \\
\hline J231546.5-590313 & 0109463601 & $231546.5-590313.6$ & 8.13 & $1.31 \pm 0.22$ & $-0.54_{-0.14}^{+0.14}$ & $-0.07_{-0.26}^{+0.26}$ & AGN2 \\
\hline $\mathrm{J} 231553.0-423800$ & 0093640701 & $231553.0-423800.4$ & 4.46 & $1.17 \pm 0.11$ & $-0.84_{-0.05}^{+0.14}$ & $-0.18_{-0.23}^{+0.20}$ & star \\
\hline $\mathrm{J} 231601.7-424038$ & 0093640701 & $231601.7-424038.9$ & 5.87 & $1.34 \pm 0.12$ & $-0.62_{-0.07}^{+0.07}$ & $-0.62_{-0.14}^{+0.13}$ & \\
\hline $\mathrm{J} 231658.8-423853$ & 0093640701 & $231658.9-423853.6$ & 9.61 & $2.46 \pm 0.18$ & $-0.45_{-0.06}^{+0.06}$ & $-0.72_{-0.08}^{+0.08}$ & \\
\hline $\mathrm{J} 233325.7-152240$ & 0093550401 & $233325.8-152240.2$ & 6.45 & $2.87 \pm 0.13$ & $-0.87_{-0.02}^{+0.02}$ & $-0.64_{-0.13}^{+0.12}$ & star \\
\hline J233421.9-151219 & 0093550401 & $233421.9-151219.0$ & 11.23 & $1.33 \pm 0.12$ & $-0.46_{-0.08}^{+0.08}$ & $-0.29_{-0.15}^{+0.13}$ & AGN1 \\
\hline $\mathrm{J} 235032.3+363156$ & 0100241001 & $235032.4+363156.9$ & 12.23 & $2.05 \pm 0.22$ & $-0.80_{-0.07}^{+0.06}$ & $-0.38_{-0.26}^{+0.15}$ & star \\
\hline $\mathbf{J} 235036.9+362204^{d}$ & 0100241001 & $235037.0+362205.0$ & 11.84 & $2.39 \pm 0.24$ & $\begin{array}{r}-0.37_{-0.09}^{+0.09} \\
-\end{array}$ & $-0.59_{-0.13}^{+0.12}$ & $\mathrm{BL}$ \\
\hline
\end{tabular}

Columns are as follows: (1) Source name; (2) XMM-Newton Observation number; (3) Right Ascension and Declination (J2000) of the source (X-ray position); (4) Angular distance (in arcmin) between the source and the MOS2 image center; (5) Source count rate, and $1 \sigma$ error, in the $0.5-4.5 \mathrm{keV}$ energy band (units of $10^{-2} \mathrm{cts} / \mathrm{s}$ ). In Table 1 we have reported the MOS 2 conversion factors between the $0.5-4.5 \mathrm{keV}$ count rate and the flux as a function of the energy spectral index, the hardness ratio HR2 and the blocking filter; (6) and (7) Hardness ratios computed as described in Sect. 3.3. The errors on the hardness ratios have been evaluated using simulations and correspond to $1 \sigma ;(8)$ Optical spectroscopic classification (AGN1: broad line AGN; AGN2: narrow line AGN; GAL: Optically Normal Galaxy; CL: Cluster of Galaxies; BL: BL Lac Object; star: star; ?: Tentative classification; see Sect. 3.2 for details).

NOTE - For two sources (XBS J052155.0-252220 and XBS J213840.5-424241) the hardness ratio HR3 is undefined since these sources are undetected above $2 \mathrm{keV}$.

${ }^{a}$ A detailed X-ray and optical spectral analysis of these sources have been reported in Caccianiga et al. (2004).

${ }^{b}$ A detailed X-ray and optical spectral analysis of these sources have been reported in Severgnini et al. (2003).

${ }^{c}$ These sources are more extended than the XMM-Newton EPIC MOS2 Point Spread Function at their off-axis angle. Count rates have been evaluated using aperture photometry.

${ }^{d}$ A detailed X-ray spectral analysis of these sources have been reported in Galbiati et al. (2004). 
Table 4. Basic information on the XMM-Newton HBSS sample.

\begin{tabular}{|c|c|c|c|c|c|c|c|}
\hline $\begin{array}{l}\text { Name } \\
\text { XBS... } \\
(1)\end{array}$ & Obs. ID & RA; Dec (J2000) & $\begin{array}{r}\text { OffAxis } \\
\operatorname{arcmin} \\
(4)\end{array}$ & $\begin{array}{r}\text { Rate } \\
\times 10^{-3} \mathrm{cts} / \mathrm{s} \\
(5)\end{array}$ & $H R 2$ & $H R 3$ & Class \\
\hline $\mathrm{J} 002618.5+105019^{b}$ & 0001930101 & $002618.5+105019.3$ & 9.56 & $2.35 \pm 0.55$ & $-0.53_{-0.04}^{+0.04}$ & $-0.67_{-0.06}^{+0.06}$ & AGN1 \\
\hline $\mathrm{J} 013240.1-133307^{b, e}$ & 0084230301 & $013240.1-133307.8$ & 11.90 & $3.23 \pm 0.71$ & $-0.02_{-0.10}^{+0.10}$ & $-0.37_{-0.11}^{+0.11}$ & AGN2 \\
\hline J013944.0-674909 ${ }^{b}$ & 0032140401 & $013944.0-674909.4$ & 2.69 & $2.05 \pm 0.46$ & $-0.50_{-0.07}^{+0.07}$ & $-0.43_{-0.13}^{+0.13}$ & AGN1? \\
\hline $\mathrm{J} 014100.6-675328^{b}$ & 0032140401 & $014100.7-675329.0$ & 6.64 & $70.95 \pm 3.84$ & $-0.45_{-0.02}^{+0.02}$ & $-0.38_{-0.03}^{+0.03}$ & star \\
\hline $\mathrm{J} 015957.5+003309^{b}$ & 0101640201 & $015957.5+003309.7$ & 9.56 & $3.80 \pm 0.92$ & $-0.63_{-0.05}^{+0.05}$ & $-0.51_{-0.10}^{+0.03}$ & AGN1 \\
\hline $\mathrm{J} 021640.7-044404^{b, e}$ & 0112371701 & $021640.7-044404.9$ & 9.35 & $2.08 \pm 0.49$ & $-0.72_{-0.05}^{+0.04}$ & $-0.26_{-0.13}^{+0.10}$ & AGN1 \\
\hline $\mathrm{J} 021808.3-045845^{b}$ & 0112371001 & $021808.3-045845.7$ & 2.26 & $2.67 \pm 0.25$ & $-0.65_{-0.02}^{+0.02}$ & $-0.52_{-0.04}^{+0.04}$ & AGN1 \\
\hline $\mathrm{J} 021817.4-045113^{b}$ & 0112371001 & $021817.4-045113.3$ & 9.58 & $3.30 \pm 0.40$ & $-0.48_{-0.03}^{+0.02}$ & $-0.56_{-0.04}^{-0.04}$ & AGN1 \\
\hline $\mathrm{J} 021822.2-050615^{a, b, d}$ & 0112371001 & $021822.3-050615.7$ & 8.48 & $4.54 \pm 0.43$ & $0.65_{-0.09}^{+0.09}$ & $0.40_{-0.07}^{+0.04}$ & AGN2 \\
\hline $\mathrm{J} 023713.5-522734^{b}$ & 0098810101 & $023713.5-522734.4$ & 12.07 & $3.23 \pm 0.61$ & $-0.57_{-0.03}^{+0.09}$ & $-0.58_{-0.06}^{+0.07}$ & AGN1 \\
\hline $\mathrm{J} 030206.8-000121^{b}$ & 0041170101 & $030206.9-000121.2$ & 11.90 & $3.10 \pm 0.42$ & $-0.53_{-0.03}^{+0.03}$ & $-0.46_{-0.06}^{+0.00}$ & AGN1 \\
\hline $\mathrm{J} 030614.1-284019^{b}$ & 0042340501 & $030614.2-284019.9$ & 10.87 & $4.61 \pm 0.92$ & $-0.49_{-0.05}^{+0.05}$ & $-0.35_{-0.09}^{+0.00}$ & AGN1 \\
\hline $\mathrm{J} 031015.5-765131^{b}$ & 0122520201 & $031015.6-765131.5$ & 5.90 & $4.39 \pm 0.46$ & $-0.46_{-0.03}^{+0.03}$ & $-0.53_{-0.04}^{+0.04}$ & AGN1 \\
\hline $\mathrm{J} 031146.1-550702^{b}$ & 0110970101 & $031146.1-550702.5$ & 12.38 & $5.87 \pm 1.12$ & $-0.58_{-0.05}^{+0.05}$ & $-0.39_{-0.11}^{+0.11}$ & AGN2 \\
\hline $\mathrm{J} 031859.2-441627^{b, d}$ & 0105660101 & $031859.3-441627.6$ & 11.55 & $2.16 \pm 0.49$ & $-0.21_{-0.08}^{+0.08}$ & $-0.47_{-0.10}^{+0.10}$ & AGN1 \\
\hline $\mathrm{J} 033845.7-352253^{a, b}$ & 0055140101 & $033845.8-352253.4$ & 10.49 & $2.37 \pm 0.36$ & $-0.05_{-0.12}^{+0.12}$ & $0.27_{-0.11}^{+0.10}$ & AGN2 \\
\hline $\mathrm{J} 040658.8-712457^{a, b}$ & 0111970301 & $040658.9-712457.7$ & 12.55 & $3.41 \pm 0.68$ & $0.39_{-0.19}^{+0.12}$ & $0.20_{-0.16}^{+0.11}$ & AGN2 \\
\hline $\mathrm{J} 040758.9-712833^{a, b}$ & 0111970301 & $040759.0-712833.5$ & 12.12 & $4.96 \pm 0.91$ & $0.49_{-0.17}^{+0.17}$ & $0.33_{-0.13}^{+0.10}$ & AGN2 \\
\hline $\mathrm{J} 041108.1-711341^{b}$ & 0111970301 & $041108.1-711341.1$ & 10.52 & $2.20 \pm 0.42$ & $-0.53_{-0.08}^{+0.08}$ & $-0.04_{-0.16}^{+0.13}$ & AGN1 \\
\hline J050536.6-290050 & 0111160201 & $050536.7-290050.8$ & 12.43 & $2.19 \pm 0.44$ & $-0.27_{-0.05}^{+0.05}$ & $-0.47_{-0.07}^{+0.07}$ & \\
\hline $\mathrm{J} 052108.5-251913^{e}$ & 0085640101 & $052108.5-251913.1$ & 3.17 & $2.11 \pm 0.55$ & $-0.59_{-0.05}^{+0.05}$ & $-0.55_{-0.09}^{+0.01}$ & AGN1 \\
\hline $\mathrm{J} 052128.9-253032^{a}$ & 0085640101 & $052128.9-253032.4$ & 10.73 & $3.08 \pm 0.88$ & $1.00_{-0.17}^{+0.00}$ & $-0.32_{-0.15}^{+0.15}$ & \\
\hline $\mathrm{J} 074202.7+742625$ & 0123100101 & $074202.7+742625.8$ & 10.86 & $3.38 \pm 0.49$ & $-0.59_{-0.03}^{+0.03}$ & $-0.28_{-0.07}^{+0.07}$ & AGN1 \\
\hline J074312.1+742937 & 0123100101 & $074312.1+742937.4$ & 5.33 & $10.92 \pm 0.64$ & $-0.61_{-0.01}^{+0.01}$ & $-0.56_{-0.02}^{+0.02}$ & AGN1 \\
\hline $\mathrm{J} 080411.3+650906$ & 0094400301 & $080411.4+650906.2$ & 9.49 & $2.41 \pm 0.50$ & $0.06_{-0.09}^{+0.09}$ & $-0.51_{-0.09}^{+0.02}$ & \\
\hline $\mathrm{J} 083737.1+254751$ & 0025540301 & $083737.2+254751.1$ & 10.61 & $7.30 \pm 1.12$ & $-0.47_{-0.04}^{+0.09}$ & $-0.53_{-0.06}^{+0.09}$ & AGN1 \\
\hline $\mathrm{J} 083737.0+255151$ & 0025540301 & $083737.1+255151.2$ & 12.25 & $2.98 \pm 0.71$ & $-0.34_{-0.08}^{+0.07}$ & $-0.52_{-0.10}^{+0.10}$ & AGN2 \\
\hline J091828.4+513931 & 0084230601 & $091828.5+513931.3$ & 6.92 & $3.03 \pm 0.54$ & $0.31_{-0.10}^{+0.09}$ & $-0.39_{-0.09}^{+0.10}+$ & AGN1 \\
\hline J095218.9-013643 & 0065790101 & $095219.0-013643.1$ & 11.78 & $24.50 \pm 2.98$ & $-0.77_{-0.03}^{+0.03}$ & $0.41_{-0.09}^{+0.09}$ & AGN1 \\
\hline $\mathrm{J} 101850.5+411506$ & 0028740301 & $101850.6+411506.6$ & 10.32 & $2.16 \pm 0.49$ & $-0.69_{-0.03}^{+0.03}$ & $-0.51_{-0.08}^{+0.09}$ & AGN1 \\
\hline J101922.6+412049 & 0028740301 & $101922.6+412049.7$ & 9.06 & $2.46 \pm 0.47$ & $-0.44_{-0.04}^{+0.04}$ & $-0.58_{-0.05}^{+0.06}$ & AGN1 \\
\hline $\mathrm{J} 104026.9+204542$ & 0059800101 & $104026.9+204543.0$ & 9.32 & $8.70 \pm 1.15$ & $-0.58_{-0.03}^{+0.04}$ & $-0.48_{-0.05}^{+0.05}$ & AGN1 \\
\hline $\mathrm{J} 104522.1-012843$ & 0125300101 & $104522.1-012843.3$ & 12.94 & $3.00 \pm 0.67$ & $-0.63_{-0.04}^{+0.04}$ & $-0.46_{-0.08}^{+0.08}$ & AGN1 \\
\hline J104912.8+330459 & 0055990201 & $104912.8+330459.8$ & 10.46 & $2.06 \pm 0.51$ & $-0.45_{-0.06}^{+0.06}$ & $-0.54_{-0.10}^{+0.09}$ & AGN1? \\
\hline J110050.6-344331 & 0112880201 & $110050.6-344332.0$ & 12.71 & $5.12 \pm 0.69$ & $-0.48_{-0.04}^{+0.06}$ & $-0.33_{-0.07}^{+0.10}+$ & \\
\hline $\mathrm{J} 112026.7+431520^{a}$ & 0107860201 & $112026.7+431520.3$ & 4.58 & $2.32 \pm 0.37$ & $0.67_{-0.08}^{+0.08}$ & $-0.32_{-0.09}^{+0.09}$ & AGN2 \\
\hline $\mathrm{J} 113106.9+312518$ & 0102040201 & $113107.0+312518.2$ & 11.18 & $2.27 \pm 0.44$ & $-0.53_{-0.08}^{+0.08}$ & $-0.15_{-0.14}^{+0.14}$ & AGN1 \\
\hline $\mathrm{J} 113121.8+310252$ & 0102040201 & $113121.8+310252.6$ & 11.58 & $3.88 \pm 0.69$ & $-0.03_{-0.07}^{+0.07}$ & $-0.35_{-0.08}^{+0.08}$ & AGN2 \\
\hline $\mathrm{J} 113148.7+311358$ & 0102040201 & $113148.7+311358.8$ & 8.45 & $3.43 \pm 0.48$ & $0.10_{-0.07}^{+0.06}$ & $-0.43_{-0.07}^{+0.07}$ & AGN2 \\
\hline $\mathrm{J} 122656.5+013126$ & 0110990201 & $122656.6+013126.2$ & 5.85 & $3.07 \pm 0.54$ & $-0.11_{-0.09}^{+0.09}$ & $-0.34_{-0.10}^{+0.10}$ & AGN2? \\
\hline J123600.7-395217 & 0006220201 & $123600.7-395218.0$ & 5.62 & $2.66 \pm 0.29$ & $-0.77_{-0.01}^{+0.01}$ & $-0.77_{-0.02}^{+0.02}$ & star \\
\hline $\mathrm{J} 124641.8+022412$ & 0051760101 & $124641.8+022412.1$ & 2.78 & $2.22 \pm 0.49$ & $-0.58_{-0.05}^{+0.05}$ & $-0.48_{-0.09}^{+0.09}$ & AGN1 \\
\hline $\mathrm{J} 132038.0+341124$ & 0093640401 & $132038.1+341124.3$ & 3.10 & $2.89 \pm 0.40$ & $-0.43_{-0.04}^{+0.04}$ & $-0.48_{-0.06}^{+0.06}$ & AGN1 \\
\hline J133942.6-315004 & 0035940301 & $133942.6-315004.8$ & 11.91 & $3.52 \pm 0.51$ & $-0.36_{-0.06}^{+0.04}$ & $-0.20_{-0.08}^{+0.06}$ & AGN1? \\
\hline $\mathrm{J} 134656.7+580315^{a}$ & 0112250201 & $134656.7+580315.4$ & 11.08 & $3.33 \pm 0.56$ & $0.46_{-0.10}^{+0.10}$ & $-0.09_{-0.10}^{+0.10}$ & GAL \\
\hline $\mathrm{J} 134749.9+582111$ & 0112250201 & $134749.9+582111.0$ & 8.53 & $7.39 \pm 0.66$ & $-0.60_{-0.02}^{+0.02}$ & $-0.54_{-0.03}^{+0.03}$ & AGN1 \\
\hline J140102.0-111224 & 0109910101 & $140102.0-111224.3$ & 9.27 & $7.21 \pm 0.59$ & $-0.52_{-0.02}^{+0.02}$ & $-0.46_{-0.03}^{+0.03}$ & AGN2? \\
\hline $\mathrm{J} 140113.4+024016^{a}$ & 0098010101 & $140113.4+024017.0$ & 12.78 & $2.10 \pm 0.52$ & $-0.55_{-0.09}^{+0.09}$ & $0.08_{-0.16}^{+0.16}$ & \\
\hline $\mathrm{J} 140127.7+025605$ & 0098010101 & $140127.7+025605.4$ & 7.25 & $6.66 \pm 0.62$ & $-0.38_{-0.02}^{+0.02}$ & $-0.53_{-0.04}^{+0.04}$ & AGN1 \\
\hline $\mathrm{J} 141531.5+113156$ & 0112250301 & $141531.6+113156.2$ & 4.15 & $2.58 \pm 0.34$ & $-0.55_{-0.03}^{+0.03}$ & $-0.47_{-0.06}^{+0.06}$ & AGN1 \\
\hline $\mathrm{J} 141830.5+251052^{c}$ & 0109960101 & $141830.5+251052.6$ & 7.58 & $7.70 \pm 1.20$ & $-0.52_{-0.04}^{+0.04}$ & $-0.65_{-0.07}^{+0.06}$ & $\mathrm{CL}$ \\
\hline $\mathrm{J} 142741.8+423335^{a}$ & 0111850201 & $142741.9+423335.8$ & 11.45 & $3.62 \pm 0.53$ & $0.48_{-0.07}^{+0.07}$ & $-0.23_{-0.07}^{+0.07}$ & \\
\hline $\mathrm{J} 143835.1+642928$ & 0111530101 & $143835.1+642928.3$ & 12.34 & $3.57 \pm 0.54$ & $0.18_{-0.07}^{+0.07}$ & $-0.37_{-0.06}^{+0.07}$ & AGN1? \\
\hline
\end{tabular}


Table 4. continued.

\begin{tabular}{|c|c|c|c|c|c|c|c|}
\hline $\begin{array}{l}\text { Name } \\
\text { XBS... } \\
\text { (1) }\end{array}$ & Obs. ID & RA; Dec (J2000) & $\begin{array}{r}\text { OffAxis } \\
\text { arcmin } \\
(4)\end{array}$ & $\begin{array}{r}\text { Rate } \\
\times 10^{-3} \mathrm{cts} / \mathrm{s} \\
(5)\end{array}$ & HR2 & $H R 3$ & Class \\
\hline $\mathrm{J} 143911.2+640526^{a}$ & 0111530101 & $143911.2+640526.8$ & 12.04 & $2.44 \pm 0.39$ & $0.86_{-0.09}^{+0.09}$ & $0.29_{-0.11}^{+0.11}$ & BL? \\
\hline $\mathrm{J} 153452.3+013104^{e}$ & 0112190401 & $153452.4+013104.6$ & 10.57 & $8.36 \pm 1.30$ & $-0.39_{-0.04}^{+0.04}$ & $-0.55_{-0.05}^{+0.11}$ & AGN1 \\
\hline J160645.9+081525 & 0067340601 & $160646.0+081525.1$ & 12.61 & $7.36 \pm 1.37$ & $0.81_{-0.08}^{+0.08}$ & $-0.23_{-0.10}^{+0.10}$ & AGN2? \\
\hline $\mathrm{J} 161820.7+124116^{a}$ & 0103461001 & $161820.7+124116.3$ & 11.56 & $2.09 \pm 0.61$ & $0.11_{-0.18}^{+0.18}$ & $-0.18_{-0.19}^{+0.18}$ & \\
\hline J165425.3+142159 & 0113070101 & $165425.4+142159.3$ & 7.44 & $5.26 \pm 1.08$ & $-0.63_{-0.03}^{+0.03}$ & $-0.61_{-0.07}^{+0.07}$ & AGN1 \\
\hline J165448.5+141311 & 0113070101 & $165448.5+141311.6$ & 12.71 & $6.25 \pm 1.79$ & $-0.54_{-0.07}^{+0.07}$ & $-0.42_{-0.13}^{+0.13}$ & AGN2 \\
\hline $\mathrm{J} 193248.8-723355^{b}$ & 0081341001 & $193248.8-723355.2$ & 8.39 & $4.47 \pm 0.73$ & $-0.23_{-0.08}^{+0.08}$ & $-0.08_{-0.10}^{+0.10}$ & AGN2? \\
\hline $\mathrm{J} 204043.4-004548^{b}$ & 0111180201 & $204043.5-004548.2$ & 10.55 & $3.24 \pm 0.80$ & $0.04_{-0.11}^{+0.11}$ & $-0.35_{-0.12}^{+0.12}$ & AGN2 \\
\hline $\mathrm{J} 205635.7-044717^{b}$ & 0112190601 & $205635.8-044717.9$ & 9.96 & $2.08 \pm 0.50$ & $-0.65_{-0.05}^{+0.04}$ & $-0.48_{-0.11}^{+0.11}$ & AGN1 \\
\hline $\mathbf{J} 205829.9-423634^{b}$ & 0081340401 & $205830.0-423635.0$ & 2.42 & $3.91 \pm 0.56$ & $-0.57_{-0.03}^{+0.03}$ & $-0.47_{-0.06}^{+0.06}$ & AGN1 \\
\hline $\mathrm{J} 213002.3-153414^{b}$ & 0103060101 & $213002.3-153414.1$ & 12.83 & $2.30 \pm 0.47$ & $-0.65_{-0.04}^{+0.03}$ & $\begin{array}{r}-0.50_{-0.09}^{+0.06} \\
+0.09\end{array}$ & AGN1 \\
\hline J213820.2-142536 & 0092850201 & $213820.2-142537.0$ & 11.17 & $2.80 \pm 0.59$ & $-0.29_{-0.07}^{+0.07}$ & $-0.45_{-0.10}^{+0.10}$ & AGN1 \\
\hline $\mathbf{J} 214041.4-234720^{b}$ & 0008830101 & $214041.5-234720.1$ & 9.80 & $3.30 \pm 0.68$ & $-0.57_{-0.05}^{+0.05}$ & $-0.39_{-0.10}^{+0.10}$ & AGN1 \\
\hline $\mathbf{J} 220601.5-015346^{b}$ & 0012440301 & $220601.5-015347.0$ & 12.92 & $2.26 \pm 0.55$ & $-0.41_{-0.06}^{+0.06}$ & $-0.41_{-0.09}^{+0.09}$ & AGN1 \\
\hline
\end{tabular}

Columns are as follows: (1) Source name; (2) XMM-Newton Observation number; (3) Right Ascension and Declination (J2000) of the source (X-ray position); (4) Angular distance (in arcmin) between the source and the MOS2 image center; (5) Source count rate, and $1 \sigma$ error, in the 4.5-7.5 keV energy band (units of $10^{-3} \mathrm{cts} / \mathrm{s}$ ). In Table 1 we have reported the MOS2 conversion factors between the 4.5-7.5 keV count rate and the flux as a function of the energy spectral index, the hardness ratio $H R 2$ and the blocking filter; (6) and (7) Hardness ratios computed as described in Sect. 3.3. The errors on the hardness ratios have been evaluated using simulations and correspond to $1 \sigma$; (8) Optical spectroscopic classification (AGN1: broad line AGN; AGN2: narrow line AGN; GAL: Optically Normal Galaxy; CL: Cluster of Galaxies; BL: BL Lac Object; star: star; ?: Tentative classification; see Sect. 3.2 for details).

NOTE $-{ }^{a}$ These 11 sources belong to the HBSS sample but not to the BSS sample, while the remaining 56 HBSS sources are present also in the BSS sample.

${ }^{b}$ A detailed X-ray and optical spectral analysis of these sources have been reported in Caccianiga et al. (2004).

${ }^{c}$ These sources are more extended than the XMM-Newton EPIC MOS2 Point Spread Function at their off-axis angle. Count rates have been evaluated using aperture photometry.

${ }^{d}$ A detailed X-ray and optical spectral analysis of these sources have been reported in Severgnini et al. (2003).

${ }^{e}$ A detailed X-ray spectral analysis of these sources have been reported in Galbiati et al. (2004). 


\section{Appendix A: Illumination Factor}

The MOS2 cameras consist of a mosaic of 7 identical, frontilluminated CCDs with a dead space between the different chips. It is clear that serendipitous X-ray sources falling close to the gaps between the CCDs (or to their edges) could have either the flux and/or the source centroid poorly determined. The poor understanding of the corrections to be applied to these sources could represent a problem in the subsequent analysis and/or interpretation of the data. It order to take into account this problem in a objective way we have used the procedure detailed below.

From the exposure map produced from the pipeline processing system we have built a mask image representing the area on the sky which is "effectively" imaged by the CCDs. To produce this mask we have used the SAS task emask with threshold1 $=0.25$ and threshold $2=0.20$ (see http: //xmm.vilspa.esa.es/external/ xmm_sw_cal/sas_frame.shtml for specific details).

Using this mask image we have thus defined the "Illumination Factor" of each source as the fraction of sky "effectively" imaged by the CCDs in a circle of 20 arcsec around the source.

The "Illumination Factor" so defined ranges between 0.3 and 1 and the lower the "Illumination Factor" the closer is the source to gaps and/or edges in the CCDs. In the BSS and/or HBSS catalogues we have retained only sources with "Illumination Factor" $\gtrsim 0.8$. Given the PSF of the MOS2 detector and its energy and off-axis dependence we have evaluated that less than $10 \%$ of the flux is lost in the case of a source with an "Illumination Factor" equal to the lower limit of 0.8 . In Table B.1 we report the complete list of sources which meet the selection criteria for the BSS and/or HBSS samples (e.g., inside the selected area between the inner and outer radius of each MOS2 image, count rate and likelihood limits, etc.) but that have been excluded from the sample because their "Illumination Factor" is below 0.8 .

Finally the produced mask has been also used, in the computation of the sky coverage, to take into account the excluded area because of edges and gaps.

\section{Appendix B: Cleaning procedure}

XMM-Newton observations are subject to "flares" in the background rate, probably due to soft protons which are collimated by the X-ray mirrors toward the EPIC cameras and interact with the structure of the detectors and the detectors itself. The current understanding is that soft protons are probably organized in clouds populating the Earth's magnetosphere.

In order to check the background quality of the dataset used we have defined a "Background Estimator Parameter" which is roughly proportional to the "real background" accumulated in the MOS2 images.

To set this "Background Estimator Parameter" for each image we have produced an histogram of the total accumulated MOS 2 counts in the 10-12 keV energy range as a function of time; the histogram bin size has been set to 100 seconds.

Thus, using this histogram we have:

a) evaluated the mean count rate $(\langle b c k\rangle)$ and its standard deviation $\left(\sigma_{b c k}\right)$

b) eliminated the time intervals which have a count rate greater than $\langle b c k\rangle+2 \times \sigma_{b c k}$

c) repeated points a) and b) 10 times

The mean count rate at the end of the loop described above is the "Background Estimator Parameter". 
Table B.1. Basic information on the sources excluded from the sample(s) since their "Illumination Factor" is less than 0.8.

\begin{tabular}{|c|c|c|c|}
\hline Obs. ID & RA; Dec (J2000) & $\begin{array}{r}\text { OffAxis } \\
\text { arcmin } \\
(3)\end{array}$ & Illum. \\
\hline 0111000101 & $001841.6+162033.4$ & 5.94 & 0.69 \\
\hline 0001930101 & $002624.9+103123.7$ & 10.77 & 0.68 \\
\hline 0112320101 & $003008.3+050340.9$ & 12.83 & 0.70 \\
\hline 0065770101 & $003241.0+394008.2$ & 5.70 & 0.42 \\
\hline 0112600601 & $012640.3+191213.0$ & 12.36 & 0.60 \\
\hline 0112630201 & 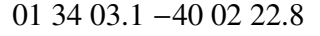 & 12.38 & 0.68 \\
\hline 0112371501 & $021821.9-043451.2$ & 7.80 & 0.48 \\
\hline 0111110501 & $022249.4-051453.0$ & 5.58 & 0.27 \\
\hline 0098810101 & $023630.3-522704.3$ & 7.66 & 0.77 \\
\hline 0098810101 & $023702.1-522348.0$ & 8.53 & 0.62 \\
\hline 0122520201 & $031314.6-765555.4$ & 5.88 & 0.71 \\
\hline 0110970401 & $031334.3-552643.6$ & 7.85 & 0.76 \\
\hline 0111970301 & $040902.1-710754.6$ & 9.83 & 0.63 \\
\hline 0085640101 & $052100.2-252852.5$ & 7.00 & 0.49 \\
\hline 0050150101 & $052557.1 \quad-334435.7$ & 10.72 & 0.74 \\
\hline 0110930101 & $061756.8+781605.3$ & 5.81 & 0.66 \\
\hline 0103860101 & $062446.8-643346.0$ & 10.80 & 0.78 \\
\hline 0112980201 & $065726.2-554953.9$ & 10.49 & 0.78 \\
\hline 0025540301 & $083827.2+255053.7$ & 5.75 & 0.65 \\
\hline 0025540301 & $083852.5+253725.3$ & 10.27 & 0.58 \\
\hline 0110660201 & $090751.1+620158.3$ & 12.77 & 0.65 \\
\hline 0084230601 & $091645.2+514145.0$ & 10.53 & 0.57 \\
\hline 0110930201 & $100056.7+554101.2$ & 8.50 & 0.69 \\
\hline 0101040301 & $102313.3+195650.5$ & 6.43 & 0.62 \\
\hline 0055990201 & $105029.0+330042.8$ & 7.63 & 0.70 \\
\hline 0110660401 & $112611.9+425245.9$ & 2.09 & $0.78^{a}$ \\
\hline 0124110101 & $122134.3+750918.1$ & 9.34 & 0.60 \\
\hline 0124110101 & $122207.3+752620.7$ & 7.88 & 0.53 \\
\hline 0092360601 & $125214.1-834642.6$ & 9.48 & 0.66 \\
\hline 0002940101 & $130632.8-233113.1$ & 12.05 & 0.62 \\
\hline 0111160101 & $133416.9+502309.6$ & 7.78 & 0.64 \\
\hline 0111160101 & $133509.6+503917.8$ & 11.58 & 0.73 \\
\hline 0111570201 & $133518.7-342145.5$ & 8.30 & 0.62 \\
\hline 0112250301 & $141539.9+112404.4$ & 5.69 & 0.37 \\
\hline 0109960101 & $141842.7+250709.5$ & 9.90 & 0.79 \\
\hline 0070740301 & $150339.6+101604.9$ & 11.46 & 0.66 \\
\hline 0018741001 & $151859.7+061839.4$ & 5.57 & 0.73 \\
\hline 0061940301 & $163239.0+781154.3$ & 5.48 & 0.56 \\
\hline 0102040101 & $172254.3+342725.3$ & 10.89 & 0.58 \\
\hline 0061940201 & $213747.7-422614.5$ & 10.55 & 0.68 \\
\hline 0008830101 & $213957.9-234535.1$ & 7.27 & 0.76 \\
\hline 0111790101 & $223606.1-260804.6$ & 6.80 & 0.55 \\
\hline 0109070401 & $224748.1 \quad-511018.1$ & 8.38 & 0.73 \\
\hline 0025541001 & $230443.5+121210.2$ & 8.04 & 0.53 \\
\hline 0123900101 & $231432.2-423301.5$ & 12.11 & 0.72 \\
\hline
\end{tabular}

Columns are as follows: (1) XMM-Newton Observation number; (2) Right Ascension and Declination (J2000) of the source (X-ray position); (3) Angular distance (in arcmin) between the source and the MOS2 image center; (4) "Illumination factor".

NOTE $-{ }^{a}$ Although this source is well contained inside the MOS2 central CCD it fall above a bad column; for this reason its "Illumination factor" is below 0.8 . 